\title{
Charged particle transport in magnetic fields in the EGSnrc Monte Carlo code system
}

\author{
by \\ Victor N. Malkov \\ A thesis submitted to the Faculty of Graduate and Postdoctoral Affairs \\ in partial fulfillment of the requirements for the degree of \\ Doctor of Philosophy \\ in \\ Physics \\ Specialization in Medical Physics \\ Ottawa-Carleton Institute for Physics \\ Department of Physics \\ Carleton University \\ Ottawa, Ontario, Canada \\ DECEMBER 2017 \\ (C) 2017 VICTOR N. MALKOV
}





\section{Abstract}

The advent of magnetic resonance guided radiation therapy provides a promising technology for dealing with tumour motion and anatomical variations during treatment. These machines possess a variety of beam energies, geometrical configurations, and different magnetic field strengths. Although photon beams do not directly experience the influence of the magnetic field, electrons set in motion will curve and impact dose distributions. Clinical reference dosimetry protocols rely on correction factors which account for the change in detector response for different beam qualities in the absence of a magnetic field. The effect of the magnetic field poses challenges for dosimetry, as ion chambers and solid state detectors respond disproportionately to the actual change in the dose to the media in the presence of the magnetic field. This necessitates an adaptation of current dosimetry protocols through calculation of high precision magnetic field and beam quality correction factors which account for detector response variation. In this work, charged particle transport in magnetic fields is implemented in EGSnrc and is shown to pass the Fano cavity test at the $0.1 \%$ level. Further good agreement with experimental ion chamber measurements is shown, and important effects such as air gaps and the unknown sensitive volume of the chamber are determined to cause several percent variation in the calculated ion chamber dose. Ion chamber magnetic field correction factors are then evaluated for over thirty cylindrical ionization chamber and a select number of parallel-plate chambers. Magnetic field correction factors for the majority of cylindrical chambers are within $1 \%$ of unity, while parallel-plate chambers require correction factors on the order of several percent and, unlike cylindrical chambers, no optimal orientation is available to reduce the effect of the magnetic field. The $\% \mathrm{dd}(10)_{x}$ beam-quality specifier is shown to have a strong dependence of the magnetic field strength, and the $\mathrm{TPR}_{10}^{20}$ is determined to be the optimal beam-quality specifier in magnetic fields. Collectively, this work contributes to the EGSnrc gold standard Monte Carlo code and to the evolving field of clinical reference dosimetry in magnetic fields. 


\section{Acknowledgments}

To my supervisor and mentor, Dr. David W. O. Rogers, I wish to extend my sincere gratitude for giving me the opportunity to work with, and learn from, him. From the first time that I met Dave, he has provided me with exceptional guidance and support. Dave's door has always been open when I needed help, and his insights and calm manner have always helped point the way forward. I have been truly lucky in my choice of supervisor and it is one of the best decisions I have ever made.

I would also like to thank all of my friends, office mates, and colleagues at Carleton University. Martin Martinov has been an instrumental help throughout my research, and provided many helpful suggestions and constantly applied his excellent programming knowledge to aide everyone in the group. Also, I am very appreciative to all of my professors who taught me about the physical details of medical physics.

To all of my loved ones and friends, I am very grateful fo your continued support and encouragements. I thank my parents who aspired to give their children a better life, and persevered through much over their multiple immigrations. This thesis is dedicated to you two.

This work has been supported by the OGSST program, QEII program, NSERC CRC (held by D.W.O. Rogers), the Faculty of Graduate and Postdoctoral Affairs, and the Physics Department. 


\section{Statement of originality}

This thesis summarizes the work of the author's academic research throughout the course of a doctoral program at Carleton University. The results presented here have been presented in peer-reviewed papers, technical reports, and conference presentations (as outlined below).

Dr. David W. O. Rogers supervised this project and provided input into all parts of the work presented. Dr. David A. Jaffray provided assistance with manuscript revision of the internal report I listed below. Aside from this, the author is responsible for the development of the magnetic field transport code, simulation set-up, analysis, and preparation and revision of manuscripts.

\section{Peer-reviewed papers}

I. V.N. Malkov and D.W.O. Rogers, "Charged particle transport in magnetic fields in EGSnrc" Medical Physics 43, 4447-4458 (2016). Selected as an Editor's choice.

- The results of this report are presented in chapter 2 and chapter 3.

II. V.N. Malkov and D. W. O. Rogers, "Sensitive volume effects on Monte Carlo calculated ion chamber response in magnetic fields" . Medical Physics 44, 48544858 (2017). Medical Physics Letter.

- The results of this report are presented in chapter 5.

III. V.N. Malkov and D.W.O. Rogers, "Monte Carlo calculated ion chamber correction factors in magnetic fields" (accepted - available on-line Dec. $7^{\text {th }}, 2017$ ).

- The results of this report are presented in chapter 6 and chapter 7 .

\section{Technical reports}

I. V.N. Malkov, D.A. Jaffray, and D.W.O. Rogers, "On magnetic field Monte Carlo dose calculations in heterogeneous lung models" CLRP-16-01 (2016).

- The results of this report are presented in chapter 4 . 


\section{Conference abstracts}

( ${ }^{*}$ indicates presenting author, ${ }^{\dagger}$ indicates an oral presentation)

I. Victor N. Malkov* and David W.O. Rogers, "Implementing magnetic and electric fields in EGSnrc", ‘International Workshop on Monte Carlo Techniques in Medical Physics, Montreal, QC, Canada, June 2014.

II. Victor N. Malkov* and David W.O. Rogers, "Improving the efficiency of charged particle transport in magnetic fields in EGSnrc", ‘IUPESM World Congress on medical physics and biomedical engineering, Toronto, ON, Canada, June 2015.

III. Victor N. Malkov* and David W.O. Rogers, "Ion chamber dose response in magnetic fields as a function of incident photon energy", 'American Association of Physicists in Medicine (AAPM) Meeting, Anaheim, California, U.S., July 2015. Med. Phys 42, 3541 (2015)

IV. David W.O. Rogers* and Victor N. Malkov, "EGSnrc calculations in a magnetic field", '‘From vision to sight: the MRI linac' symposium, Utrecht, The Netherlands, Sept. 2015.

V. Victor N. Malkov* and David W.O. Rogers, "Not all geometries are equivalent for magnetic field Fano cavity tests" ${ }^{\dagger}$ Canadian Organization of Medical Physicists (COMP) Annual Meeting, St. John's, NL, Canada, July 2016. Med. Phys 43, 4959 (2016)

VI. Victor N. Malkov* David A. Jaffrey, and David W.O. Rogers, "Lung cannot be treated as homogeneous in radiation transport simulations in B-fields", ${ }^{\dagger}$ AAPM Meeting, Washington, D.C., U.S., Aug. 2016. Med. Phys 43, 3854 (2016)

VII. Victor N. Malkov* and David W.O. Rogers, "Angular dependence of beam quality and magnetic field conversion factors", ${ }^{\dagger}$ COMP Annual Meeting (first place Young Investigator's Symposium), Ottawa, ON, Canada, July 2017. Med. Phys. 44, 4371 (2017)

VIII. Victor N. Malkov* and David W.O. Rogers, "Impact of the true sensitive volume on ion chamber response in magnetic fields", †International Workshop on Monte Carlo Techniques in Medical Physics, Naples, Italy, Oct. 2017. 


\section{Contents}

$\begin{array}{lll}\text { Abstract } & \text { ii }\end{array}$

$\begin{array}{ll}\text { Acknowledgments } & \text { iii }\end{array}$

Statement of originality iv

Table of contents viii

List of tables $\quad$ ix

List of figures $\quad$ xi

Nomenclature and notation $\quad$ xii

1 Introduction $\quad 1$

1.1 Magnetic resonance guided radiation therapy (MRgRT) . . . . . . . . 1

1.2 Reference dosimetry ..................... . . 4

1.2.1 Instrumentation - ion chambers chambers . . . . . . . . . . 4

1.2.2 TG-51 protocol . . . . . . . . . . . . . . . 6

1.2.3 The EGSnrc simulation package . . . . . . . . . . . . . 12

1.2.4 Thesis purpose ..................... 14

1.2 .5 Thesis outline . . . . . . . . . . . . . . . . . 14

2 Implementing charged particle transport in magnetic fields in EGSnrc

2.1 Lorentz force in a condensed history calculation . . . . . . . . . . . 16

2.2 Scaled $\delta_{u}$ algorithm . . . . . . . . . . . . . . . . . . . 21

2.3 Adaptive integration algorithm . . . . . . . . . . . . . . . 22

2.4 Magnetic field influence in single scatter (SS) mode . . . . . . . . . . . . 22

2.5 Boundary crossing . . . . . . . . . . . . . . . . 23 


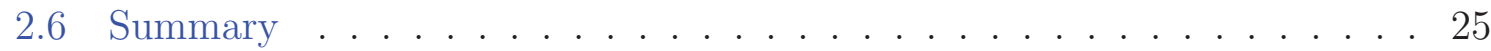

3 Validation of magnetic field transport code $\quad 26$

3.1 Fano cavity test . . . . . . . . . . . . . . . . 26

3.1.1 Results and discussion . . . . . . . . . . . . . . 29

3.2 Comparison to experimental ion chamber measurements . . . . . . . . . 33

3.2.1 Results and discussion . . . . . . . . . . . . . . . 35

3.3 Impact on timing and efficiency . . . . . . . . . . . . . . . . . 39

3.3.1 Results and discussion . . . . . . . . . . . . . . . . . 39

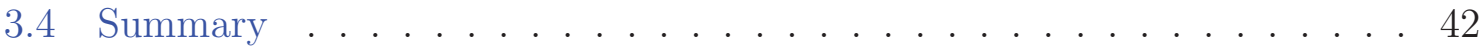

4 Magnetic field dose calculations in heterogeneous lung models 44

4.1 Introduction . . . . . . . . . . . . . . . . . . . . . . 44

4.2 Lung-like geometry configuration . . . . . . . . . . . . . . . 45

4.3 Results and discussion . . . . . . . . . . . . . . . . . . 49

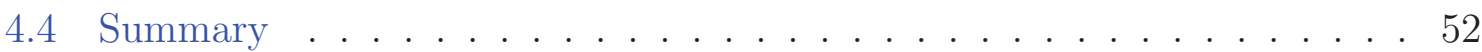

5 Sensitive volume effects on ion chamber response in magnetic fields 54

5.1 Introduction . . . . . . . . . . . . . . . . . . . . . 54

5.2 Set-up of sensitive volume simulations . . . . . . . . . . . . . 55

5.3 Results and discussion . . . . . . . . . . . . . . . . . . 57

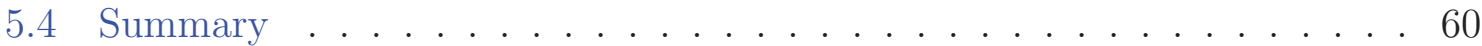

6 Optimal orientation for clinical reference dosimetry in magnetic fields

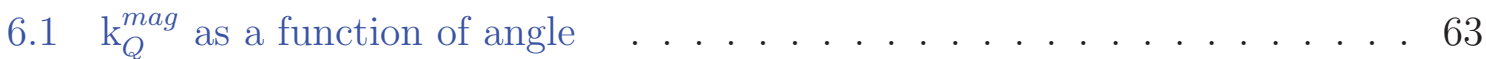

6.1 Results and Discussion . . . . . . . . . . . . 68

6.2 Sensitive volume effects as a function of angle . . . . . . . . . . . 71

6.2.1 Results and Discussion . . . . . . . . . . . . . . . 72

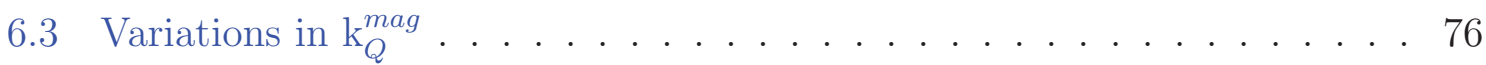

6.3.1 Results and Discussion . . . . . . . . . . . . 76

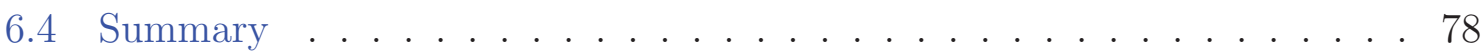

7 Ionization chamber magnetic field correction factors and additional $\begin{array}{lr}\text { considerations of magnetic field dosimetry } & 80\end{array}$

7.1 Cylindrical ion chamber models . . . . . . . . . . . . . . . . 80

7.2 Beam-quality dependence of $\mathrm{k}_{Q}^{m a g}$ and $\mathrm{k}_{B} \ldots \ldots . \ldots 2$ 
7.2.1 Results and Discussion ................ . 83

$7.3 \mathrm{k}_{Q}^{\mathrm{mag}}$ and $\mathrm{k}_{B}$ calculations for ion chambers . . . . . . . . . . . 87

7.3.1 Results and Discussion . . . . . . . . . . . . . . . . . 88

7.4 Impact of the ICRU-90 recommendation on $\mathrm{k}_{Q} \ldots \ldots$. . . . . . . . . . . . 92

7.4.1 Results and Discussion . . . . . . . . . . . . . . . . . 92

$7.5 \mathrm{k}_{Q}^{\mathrm{mag}}$ and $\mathrm{k}_{B}$ for parallel-plate chambers . . . . . . . . . . . . . 93

7.5.1 Results and Discussion . . . . . . . . . . . . . . . . . 94

7.6 Beam-quality specification . . . . . . . . . . . . . . . . 95

7.6.1 Results and Discussion . . . . . . . . . . . . . . . . 97

7.7 Summary . . . . . . . . . . . . . . . . . . 100

8 Final overview and outlook 102

8.1 Summary and conclusions . . . . . . . . . . . . . . . . 102

8.2 Future work . . . . . . . . . . . . . . 106

$\begin{array}{ll}\text { Appendices } & 107\end{array}$

$\begin{array}{ll}\text { A Normalization of final direction vector } & 108\end{array}$

$\begin{array}{ll}\text { B Condensed history } t_{\text {perp }} \text { restriction } & 111\end{array}$

$\begin{array}{ll}\text { C Energy loss over elastic mean free path } & 113\end{array}$

$\begin{array}{ll}\text { References } & 116\end{array}$ 


\section{List of Tables}

5.1 Geometric properties of ion chambers and variations in response due to changes in the sensitive volume . . . . . . . . . . 58

6.1 Percent difference in $\mathrm{k}_{Q}^{\text {mag }}$ due to a $10^{\circ}$ chamber misalignment or a change in the sensitive volume . . . . . . . . . . . . . . 77

7.1 Cylindrical ion chamber $\mathrm{k}_{Q}, \mathrm{k}_{B}$, and $\mathrm{k}_{Q}^{\operatorname{mag}}$ values for ${ }^{60} \mathrm{Co}$ and $7 \mathrm{MV}$

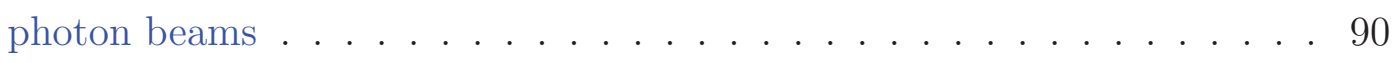

7.2 Comparison of $\mathrm{k}_{B}$ between this work and O'Brien et al. . . . . . . . 91

7.3 Comparison of ICRU-37 and ICRU-90 recommendations . . . . . . . 92

7.4 Comparison of ICRU-37 and ICRU-90 $\mathrm{k}_{Q}$ values $\ldots \ldots \ldots$

$7.5 \mathrm{k}_{Q}, \mathrm{k}_{Q}^{\mathrm{mag}}$, and $\mathrm{k}_{B}$ correction factors for parallel-plate chambers . . . . 95

7.6 Photon beam $\mathrm{TPR}_{10}^{20}$ and $\% \mathrm{dd}(10)_{x}$ values at $0 \mathrm{~T} \ldots \ldots$ 


\section{List of Figures}

1.1 Ion chamber simplified schematic . . . . . . . . . . . . . 5

1.2 Sample PDD for 7 MV photon beam . . . . . . . . . . . . . 8

1.3 Tissue phantom ratio measurement set-up . . . . . . . . . . . . . 10

2.1 Simplified EGSnrc condensed history step . . . . . . . . . . . . . 17

2.2 Magnetic field boundary crossing algorithm . . . . . . . . . . . . . . 24

3.1 Fano test geometries . . . . . . . . . . . . . . . . . 27

3.2 No magnetic field slab Fano test results . . . . . . . . . . . . . . . . . . 30

3.3 Magnetic field 2 mm slab Fano test results . . . . . . . . . . . . . . . . 31

3.4 Magnetic field $2 \mathrm{~cm}$ slab Fano test results . . . . . . . . . . . . . . . 31

3.5 Magnetic field ion chamber Fano test results . . . . . . . . . . . . . . . 32

3.6 Slab Fano test as a function of magnetic field . . . . . . . . . . . . . 33

3.7 Geometric set-up of Config. I and II of the NE 2571 in Delrin (validation) 35

3.8 Config. I NE 2571 response as a function of magnetic field strength . . . 36

3.9 Config. II NE 2571 response as a function of magnetic field strength . . . 36

3.10 Config. I NE 2571 reponse air gaps around the chamber . . . . . . . . . . 38

3.11 Config. II NE 2571 response with air gaps around the chamber . . . . . . 38

3.12 NE 2517 (Config. I) relative timing and efficiency as a function of $\delta_{u}$. . 40

3.13 NE 2517 (Config. I) $\varepsilon_{\text {norm }}$ and $t_{\text {norm }}$ as a function of magnetic field strength 41

4.1 Homogeneous and heteogeneous simplified lung models . . . . . . . . . . 47

4.2 Heterogenous lung tube geometry . . . . . . . . . . . . . . . . . 48 
4.3 Slab lung geometry homogeneous to heterogeneous dose ratio . . . . . . . 51

4.4 Tube lung geometry homogeneous to heterogeneous dose ratio . . . . . . 52

5.1 Ion chamber simulation geometry for sensitive volume study . . . . . . . 57

5.2 Effect of sensitive volume on magnetic field chamber reponse . . . . . . . 62

6.1 Geometric set-up for correction factor calculations . . . . . . . . . . . . . 64

6.2 Beam's eye view of rotating chamber orientations . . . . . . . . . . 65

6.3 Ion chamber orientations in magnetic fields (definition of CI-CV) . . . . 67

6.4 Ion chambers used for angular magnetic field correction factor simulations 68

$6.5 \mathrm{k}_{Q}^{\mathrm{mag}}$ as a function of angle for six chambers . . . . . . . . . . 70

6.6 Sensitive volume segmentation for $\mathrm{k}_{Q}^{\mathrm{mag}}$ caluclations . . . . . . . . . 72

6.7 Chamber response as a function of chamber length . . . . . . . . . 73

$6.8 \mathrm{k}_{Q}^{m a g}$ as a function of angle and different sensitive volumes $\ldots . . . .75$

7.1 EGSnrc simulation comparison results from Spindeldreier et al. . . . . . . 82

7.2 Values $\mathrm{k}_{Q}^{m a g}$ as a function of $\% \operatorname{dd}(10)_{x}$ for the $0 \mathrm{~T}$ field . . . . . . . . 85

$7.3 \mathrm{k}_{B}$ as a function of beam quality for cylindrical ion chambers $(1.5 \mathrm{~T})$. 86

7.4 $\mathrm{k}_{B}$ as a function of beam quality for cylindrical ion chambers $(0.35 \mathrm{~T})$. 87

7.5 DDCs for ${ }^{60} \mathrm{Co}, 7 \mathrm{MV}$, and $25 \mathrm{MV}$ photon spectra for $0 \mathrm{~T}, 1 \mathrm{~T}$, or $2 \mathrm{~T}$. 98

7.6 \% dd $(10)_{x}$ as a function of magnetic field for several photon beam spectra 99

7.7 $\mathrm{TPR}_{10}^{20}$ as a function of magnetic field for several photon beam spectra . . 100

A.1 Analytical and simulated charged particle trajectories in vacuum . . . . . 109

B.1 Condenced history boundary step-size restriction . . . . . . . . . . . 111

C.1 Electron mean free path as a function of energy . . . . . . . . . . . 114

C.2 Percent energy loss per mean free path as a function of energy . . . . . 115 


\section{Nomenclature and notation}

$\delta_{u} \quad$ Magnetic field step-size restriction based on the fractional change of the particle direction due to the magnetic field

$\|_{c h} \quad$ Orientation in which the magnetic field is parallel to the long-axis of the ion chamber and perpendicular to the photon beam

$\|_{p h} \quad$ Orientation in which the magnetic field is perpendicular to the long-axis of the ion chamber and parallel to the photon beam

\%dd(10) $)_{x} \quad$ Photon component percent depth dose value at $10 \mathrm{~cm}$

3-PI, 1-PI Three and one point integration (numerical)

BCA Boundary crossing algorithm

CBCT Conebeam computed tomography

CH Condenced history technique

DDC Depth dose curve

ECUT Electron total energy cut-off $[\mathrm{MeV}]$

ERE Electron return effect

ESTEPE EGSnrc parameter to control the maximum fractional energy loss per step

FFF Flatening filter free

IGRT Image guided radiation therapy

$\mathbf{k}_{B} \quad$ Magnetic field correction factor

$\mathbf{k}_{Q} \quad$ Beam-quality correction factor

$\mathbf{k}_{Q}^{\text {mag }} \quad$ Beam-quality and magnetic field correction factor

M Ion chamber charge reading

MC Monte Carlo (simulation algorithm)

MRgRT Magnetic resonance guided radiation therapy

MRI Magnetic resonance imaging

$\mathbf{N}_{D, w}^{60}$ Co $\} \quad$ Absorbed dose-to-water calibration coefficient for a reference cobalt-60 beam

PCUT Photon total energy cut-off $[\mathrm{MeV}]$

PDD Percent-depth-dose

PP Parallel-plate ionization chamber

Response Average dose to sensitive chamber volume per unit incident photon fluence.

Relative response is as response but normalized by the $0 \mathrm{~T}$ simulation.

SS Single scatter transportation algorithm

SSD Source to surface distance

$\mathbf{T P R}_{10}^{20} \quad$ Tissue phantom ratio at $10 \mathrm{~cm}$ and $20 \mathrm{~cm}$ depth 


\section{Chapter 1}

\section{Introduction}

This chapter outlines the basic theory of magnetic resonance guided radiation therapy and the dosimetry challenges in such systems. A brief overview of clinical reference dosimetry and how the magnetic fields effects can be included in dosimetry protocols is given.

\subsection{Magnetic resonance guided radiation therapy (MRgRT)}

The Canadian Cancer Society ${ }^{1}$ reports that roughly 1 in 2 Canadians will develop a form of cancer over their lifetime. Improvements in lifestyle habits, detection methods, and treatment are attributed to the continued reduction in the age-standardized mortality rate. Cancer treatment is based on a combination of surgical methods, chemotherapy, and radiation therapy. The main goal of radiation therapy is to deliver a sufficiently

high dose (energy deposited per unit mass $[\mathrm{J} / \mathrm{kg}]$ ) to the tumour that will induce cellular death while mitigating exposure of healthy tissues surrounding the tumour.

Development of treatments such as volumetric arc therapy ${ }^{2}$ and helical tomother$a^{3}{ }^{3}$ has permitted the delivery of radiation treatment plans which conform to the tumour geometry by allowing beam delivery from a large number of angles. Such machines also allow for dose to healthy tissue be reduced and highly attenuating regions, like bone, be avoided. However, as intricate as these machines and planning systems 
become, patient motion and geometrical changes during treatment cannot be entirely eliminated and typically a planned treatment volume is assigned which adds a margin surrounding the tumour. This larger treatment volume leads to increased dose delivery to the region surrounding the tumour and may include healthy tissue which could be avoided if the true position of the tumour was available. This motivates the use of image guided radiation therapy (IGRT) machines, and a large majority of the currently available technologies rely on the use of x-ray or cone-beam computed tomography (CBCT) imaging $^{4}$. An example of such a system is the Cyberknife ${ }^{5}$ which uses two x-ray panels to correlate tumour position with patient breathing and motion patterns to guide the robotic arm on which the linear accelerator is mounted.

Magnetic resonance imaging (MRI) provides excellent soft tissue contract which is lacking in x-ray and CBCT imaging systems. Further, the absence of additional imaging dose makes MRI an ideal candidate for IGRT. Using MRI for image guidance can also avoid the application of fiducial markers, used for tracking in x-ray based systems, and provide an avenue for monitoring biological response using functional imaging. The first magnetic resonance guided radiation therapy ${ }^{6}$ (MRgRT) machine was made available by View-Ray and consists of a $0.35 \mathrm{~T}$ MRI and three ${ }^{60} \mathrm{Co}$ sources. The advantage of using isotope sources is that beam quality calibration is not required since the spectrum is well studied, and, once the activity of the source is known, the treatment duration can be adjusted to reflect source decay. However, the lower-energy photons of the ${ }^{60} \mathrm{Co}$ sources are unable to penetrate as deep into tissue as a high-energy linear accelerator source, and these machines, and their future iterations, are being accordingly updated with roughly $6 \mathrm{MV}$ photon sources. Similarly, Elekta is developing and testing an MRgRT machine with a $1.5 \mathrm{~T}$ MRI and a $7 \mathrm{MV}$ linear accelerator ${ }^{7-9}$. In both of these machines, the magnetic field is perpendicular to the incoming photon beams, and a number of groups are also developing MRgRT implementations, with some configured with the magnetic field parallel to the photon beam ${ }^{10-12}$. 
The presence of the magnetic field during treatment in MRgRT machines does not impact photons. However, charged particles knocked into motion by the incoming photon beam through atomic interactions, such as photoelectric effect and Compton scattering, will have their trajectories altered based on the Lorentz force. In the absence of a magnetic field, charged particles will, for the most part, travel linearly between scattering events, which will be much closer together in higher density media (as compared to air). When a magnetic field is present, these linear paths will be altered into curved trajectories. Due to the larger distance between interaction in low density media the magnetic field induced deflection will be more pronounced than in high density materials. This leads to charged particles being able to curve back to high density surfaces at interfaces between low and high density media. Consequently, these returning particles will produce an increase in surface dose in the higher density material $^{13-16}$, as compared to when no magnetic field is present. This effect is called the electron-return-effect (ERE), and the consequence of electrons being swept to a particular region is that another part of the geometry will observe a reduction in impinging electrons and therefore see a reduction in the local dose. Additional perturbations in photon beams such as dose enhancement and reductions in penumbra dose have been established $^{7,17,18}$. In the context of patient treatment planning, the presence of the magnetic field can produce hot and cold spots in the dose distribution, particularly near lung-tissue or tissue-air interfaces. Further, as will be seen in subsequent sections, ion chambers, used for calibrating linear accelerators, can see several percent change in their reading when a magnetic field is present.

The effects of the magnetic field on dose distributions and detector systems ${ }^{12,19,20}$ motivates the development of Monte Carlo (MC) codes capable of accounting for the presence of the magnetic field. MC simulations are often clinically used to perform inverse planning for patient treatments, and by taking into account the magnetic field influence, such algorithms could optimize the dose distribution and potentially take ad- 
vantage of the Lorentz force. Full MC codes are the basis of the calculation of correction factors, as is described below, for use in clinical reference ion chamber dosimetry.

\section{$1.2 \quad$ Reference dosimetry}

The aim of clinical reference dosimetry is to allow users to apply national dose-to-water standards to calibrate linear accelerators. TRS- $398^{21}$ and TG-5122,23 provide protocols for performing reference dosimetry in high-energy photon and electron beams. These protocols are based on the use of correction factors applied to measurements made by calibrated ion chambers. This work focuses on TG-51, however the magnetic field correction factors calculated here are transferable to other dosimetry protocols.

\subsubsection{Instrumentation - ion chambers chambers}

Ionization chambers are at the core of clinical reference dosimetry. These instruments are air filled chambers which use an applied potential within the air volume to collect charges released due to high-energy particles traversing the region. These detectors are chosen based on their sensitivity, long term stability (constant current reading for the same dose rate), and instant readout (as compared to other dosimetry systems which require processing after radiation exposure) ${ }^{22}$. One type of ion chambers is the cylindrical chamber design, and a simplified schematic is given in Fig. 1.1a. This chamber is used by setting up the central axis of the chamber to be perpendicular to the incoming radiation beam. An electric potential is set-up between the central electrode and the wall of the chamber. The electric field produced by this potential can be seen in Fig. 1.1a, and a slight fringing of the field is shown near the guard electrode. The purpose of the guard electrode is to ground and minimize leakage current that would impact the current readout by the central electrode, and materials in the stem are chosen to provide additional electric insulation. Chambers from different manufacturers carry 
the main features highlighted in Fig. 1.1a, and, when creating MC models of these chambers, the air volume in which the dose is scored is often the geometric sensitive volume. This is a close approximation to the true collection volume which, as will be seen in Chapter 5, is smaller than the geometric sensitive volume due to the central electrode being unable to collect charges from the region in which the guard electrode causes fringing of the electric field. The cylindrical ion chamber is the recommended type for performing clinical reference dosimetry ${ }^{22}$, but parallel-plate $(\mathrm{PP})$ chambers are still used for certain dosimetry applications. The simplified design of the PP chamber is given in Fig. 1.1b, and this chamber is used by aligning the central axis of the chamber to be parallel with the incoming radiation beam. The window, which faces towards the radiation beam, is the electrode pair to the collection electrode, and again a guard electrode is used to minimize the leakage current and to minimize scatter from the side walls entering the sensitive volume. The sensitive volume sits between the collection electrode and the window, and the electric field in this region is fairly uniform since any fringing occurs in the insensitive volume of the chamber.

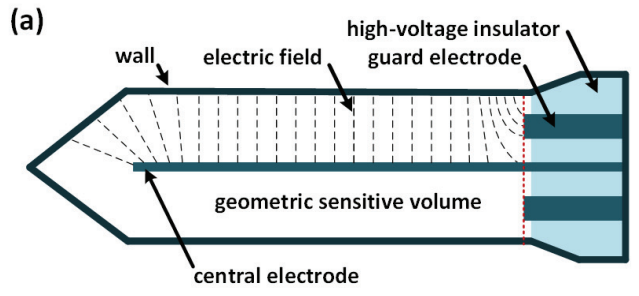

(b)

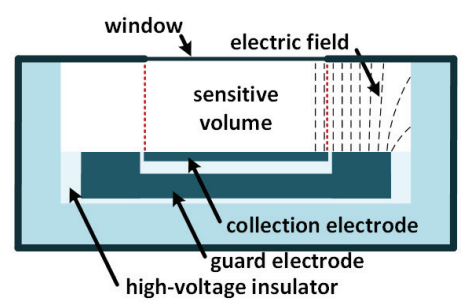

Figure 1.1: Simplified cylindrical (left) and parallel-plate (right) ionization chamber schematic. The dashed lines represent the charge collecting electric field.

Ion chambers produce a charge reading in units of coulombs, and this raw reading is corrected for several physical effects. These effects include temperature and pressure variations, ion recombination, leakage current, and polarity of the applied potential. The details of these corrections are found in the TG-51 protocol and the addendum, and are not addressed here since this work deals with the calculation of magnetic field correction factors as will be seen in the subsequent section. 


\subsubsection{TG-51 protocol}

Clinical dosimetry of radiation sources is performed under protocol defined reference conditions using ion chambers which have absorbed dose-to-water calibration coefficients traceable to national standards. Per the TG-51 protocol ${ }^{22}$, the ion chamber corrected charge reading, M, should be obtained with the ion chamber positioned at a $10 \mathrm{~cm}$ depth at the centre of a $30 \times 30 \times 30 \mathrm{~cm}^{3}$ water phantom. The incoming radiation beam should have a source to surface distance $(\mathrm{SSD})$ of $100 \mathrm{~cm}$ and produce a field size at the surface of the water phantom of $10 \times 10 \mathrm{~cm}^{2}$. The dose to the air volume of the chamber, $D_{a i r}^{Q}$, can be calculated using

$$
D_{a i r}^{Q}=\frac{M}{m_{a i r}}\left(\frac{W}{e}\right)_{a i r}
$$

where $m_{\text {air }}$ is the mass of the sensitive air volume of the chamber and $\left(\frac{W}{e}\right)_{\text {air }}$ is the average energy lost per coulomb of charge released by electrons in air (assumed to be constant as a function of beam quality). To determine the dose to water in the user's beam quality, the ion chamber must first be calibrated at a national standards laboratory (e.g., the National Research Council Canada). The chamber calibration is performed at the previously mentioned reference conditions with the only difference being that the ion chamber is positioned at a depth of $5 \mathrm{~cm}$ in the water phantom. A ${ }^{60} \mathrm{Co}$ source is used to obtain the chamber specific absorbed-dose to water calibration factor, $\mathrm{N}_{D, w}^{60}$, which is defined as:

$$
N_{D, w}^{60} C o=\frac{D_{w}^{60} C o}{M^{60} C o}
$$

where $D_{w}^{60} C o$ is the dose-to-water, determined via calorimetry, and $M^{60} C o$ is the corrected chamber reading in the ${ }^{60} \mathrm{Co}$ beam. Then the dose to water in the user's beam quality, 
Q, is obtained using,

$$
D_{w}^{Q}=M k_{Q} N_{D, w}^{60} C o
$$

where $\mathrm{k}_{Q}$ is the ion chamber specific quality conversion factor which the user would obtain from the TG-51 protocol for their specific case. This factor is defined as

$$
k_{Q}=\frac{N_{D, w}^{Q}}{N_{D, w}^{60} C o},
$$

and substituting this expression into Eq. 1.3 demonstrates how $\mathrm{k}_{Q}$ allows $\mathrm{N}_{D, w}^{60}$ to be transferred from the reference ${ }^{60} \mathrm{Co}$ to any beam quality. When using $\mathrm{MC}$ and assuming that $(W / e)$ is constant as a function of beam quality, this equation can be written as

$$
k_{Q}=\frac{\left(\frac{D_{w}}{D_{c h}}\right)^{Q}}{\left(\frac{D_{w}}{D_{c h}}\right)^{60} C o}=\left(\frac{D_{w}}{D_{c h}}\right)^{{ }^{60} C o}
$$

where $D_{w}$ is the dose-to-water in the absence of the ion chamber, and $D_{c h}$ is the dose to ion chamber sensitive volume. These dose quantities are determined at the reference depths of $5 \mathrm{~cm}$ and $10 \mathrm{~cm}$ for ${ }^{60} \mathrm{Co}$ and beam quality Q, respectively. Using ion chamber measurements and water calorimetry to obtain the dose-to-water in various beam qualities, $\mathrm{k}_{Q}$ can be experimentally determined. However, all quantities in Eq. 1.5 are $\mathrm{MC}$ calculable, and $\mathrm{k}_{Q}$ can be obtained using accurate $\mathrm{MC}$ ion chamber models and dose-to-water simulations.

\section{Beam-quality specification}

The TG-51 protocol ${ }^{22}$ and its corresponding addendum ${ }^{23}$ provide $\mathrm{k}_{Q}$ for a large number of chambers and beam qualities. To obtain the necessary $\mathrm{k}_{Q}$ value, the user is required to make a beam-quality measurement. The two most commonly used beam-quality specifiers are $\% \mathrm{dd}(10)_{x}$, the value of the photon component percent depth-dose at $10 \mathrm{~cm}$ 
of depth in water, and $\mathrm{TPR}_{10}^{20}$, the tissue phantom ratio at $10 \mathrm{~cm}$ and $20 \mathrm{~cm}$ (explained below in greater detail). A sample simulated percent depth dose (PDD) curve is shown in Fig. 1.2 and demonstrates how $\% \operatorname{dd}(10)_{x}$ can be determined from this graph. In practice the user would perform ion chamber charge measurements as a function depth and normalize to the maximum value to obtain a percent depth-ionization curve. This curve is then used to obtain $\% \operatorname{dd}(10)_{x}$ (when using cylindrical ion chambers a slight shift of the depth-ionization curve is necessary to account for the effective point of measurement of the chamber). The PDD for the $\% \operatorname{dd}(10)_{x}$ measurement is determined with an SSD of $100 \mathrm{~cm}$ and a field size of $10 \times 10 \mathrm{~cm}^{2}$ at the surface of a $30 \times 30 \times 30 \mathrm{~cm}^{3}$ water phantom.

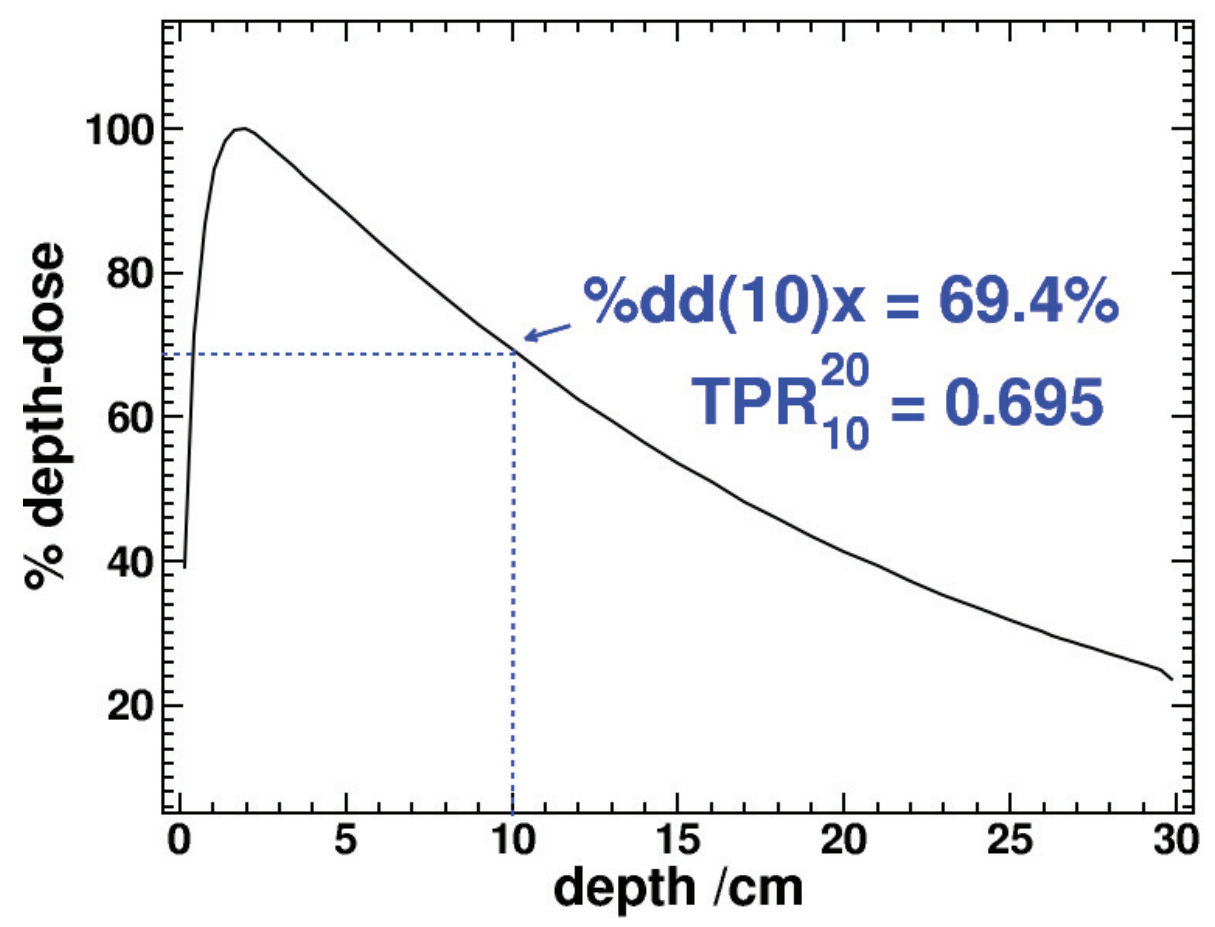

Figure 1.2: Simulated percent depth-dose curve for a $7 \mathrm{MV}$ photon beam. The value of $\% \mathrm{dd}(10)_{x}$ can be read off the graph, while $\mathrm{TPR}_{10}^{20}$ is determined using an additional simulation as shown in Fig. 1.3.

For high-energy photon beams, the TG-51 addendum introduced functional forms 
for $\mathrm{k}_{Q}$ which have the general expression

$$
k_{Q}=A+B \cdot 10^{-3} \cdot \% d d(10)_{x}+C \cdot 10^{-3} \cdot\left(\% d d(10)_{x}\right)^{2}
$$

where A, B, and C are the fit parameters which can be looked up for each recommended chamber, and $\% \mathrm{dd}(10)_{x}$ is the user measured beam-quality specifier.

An alternative beam-quality specifier, is $\mathrm{TPR}_{10}^{20}$ which is used in the TRS-398 protocol $^{21}$. The measurement of $\mathrm{TPR}_{10}^{20}$ is made by setting the field size to be $10 \times 10 \mathrm{~cm}^{2}$ at the point of measurement and maintaining a $100 \mathrm{~cm}$ source to detector distance. The first ion chamber measurement or dose calculation is made at $10 \mathrm{~cm}$ depth, $\mathrm{D}_{10}$, and the second is made at $20 \mathrm{~cm}, \mathrm{D}_{20}$. Effectively, for the second dose calculation an additional $10 \mathrm{~cm}$ of water is added to the top of the water phantom. Then $\mathrm{TPR}_{10}^{20}$ is defined as

$$
T P R_{10}^{20}=\frac{D_{20}}{D_{10}}
$$

The set-up for performing the $\mathrm{TPR}_{10}^{20}$ measurement is shown in Fig. 1.3, and the $\mathrm{TPR}_{10}^{20}$ value associated with the $7 \mathrm{MV}$ spectrum is given in Fig. 1.2. Kalach and Rogers ${ }^{24}$ have shown that $\mathrm{TPR}_{10}^{20}$ could be converted to $\% \mathrm{dd}(10)_{x}$ using a third order polynomial equation, and this conversion works well for clinical filtered photon beams. The advantage of using $\% \mathrm{dd}(10)_{x}$ is that it remains an accurate beam-quality specifier for flattening-filter-free (FFF) beams as well ${ }^{25}$. 

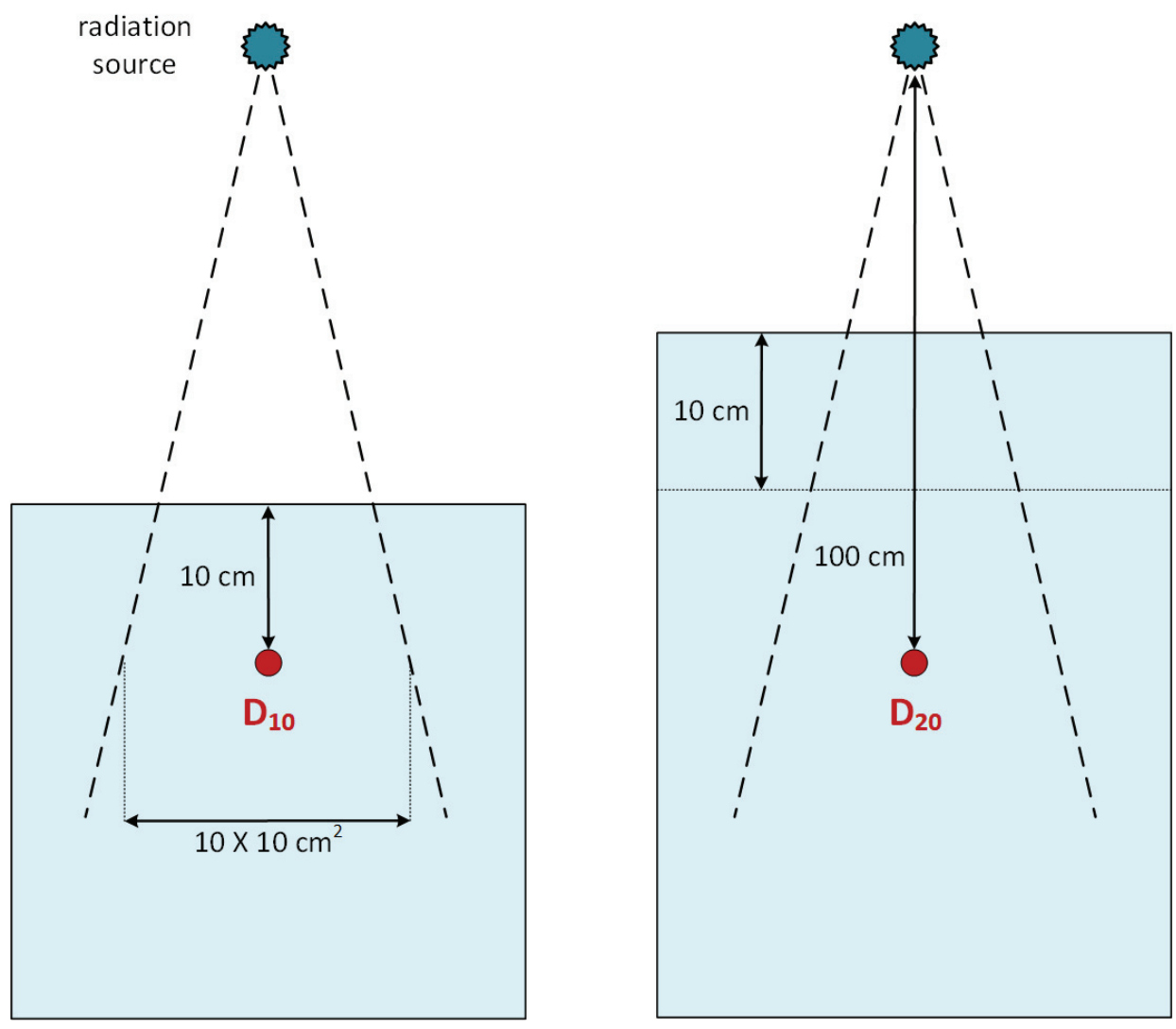

Figure 1.3: Geometric set-up for performing a $\mathrm{TPR}_{10}^{20}$ measurement or calculation. The red circle indicate the point of measurement, and $\mathrm{TPR}_{10}^{20}$ is determined by calculating the ratio of $\mathrm{D}_{20}$ and $\mathrm{D}_{10}$.

\section{Dosimetry in magnetic fields}

As noted above, the magnetic field perturbs charge particle trajectories and leads to changes in ion chamber readings. To accommodate the presence of the magnetic field, a change to Eq. 1.3 is required. One approach to obtain the dose-to-water in the desired beam quality and magnetic field, $D_{W}^{Q, B}$, is to substitute $\mathrm{k}_{Q}$ with a magnetic field and quality correction factor, $\mathrm{k}_{Q}^{\mathrm{mag}}$ :

$$
D_{W}^{Q, B}=M k_{Q}^{m a g} N_{D, w}^{60} \mathrm{Co}
$$


Here $\mathrm{k}_{Q}^{m a g}$ accounts for the change from the reference conditions of $0 \mathrm{~T}$ in a ${ }^{60} \mathrm{Co}$ beam to the desired magnetic field and beam quality in a single factor. The second approach is to introduce an additional magnetic field correction factor, $\mathrm{k}_{B}$, such that:

$$
D_{W}^{Q, B}=M k_{Q} k_{B} N_{D, w}^{60} C o
$$

Here $\mathrm{k}_{B}$ accounts only for the change in the calibration coefficient due to the magnetic field. This is the approach explored by a few groups for specific chambers, magnetic fields, and beam qualities ${ }^{26,27}$. A potential advantage of using $\mathrm{k}_{B}$ instead of $\mathrm{k}_{Q}^{\text {mag }}$ is a reduced dependence of the correction factor on beam quality, but a disadvantage is the added complexity in using two correction factors. Overall, these two factors are related by

$$
k_{B}=\frac{k_{Q}^{m a g}}{k_{Q}} .
$$

Similar to the standard method, MC calculates $\mathrm{k}_{Q}^{\operatorname{mag}}$ as:

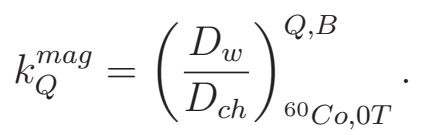

Both water and chamber doses are determined taking the point of measurement as the central axis of the cylindrical chambers.

Similarly $\mathrm{k}_{B}$ is calculated as:

$$
k_{B}=\left(\frac{D_{w}}{D_{c h}}\right)_{Q, 0 T}^{Q, B},
$$

which involves the same quantities, but all determined for the beam quality $\mathrm{Q}$ and the reference ${ }^{60}$ Co beam is not involved.

A few additional complications to clinical reference dosimetry in magnetic fields have been highlighted in the literature. O'Brien et al. ${ }^{26}$ note variations in the $\% \mathrm{dd}(10)_{x}$ 
beam-quality specifier due to the presence of the magnetic field and therefore the tissue phantom ratio at 20 and $10 \mathrm{~cm}, \mathrm{TPR}_{10}^{20}$, may be the preferred choice as the beamquality specifier in magnetic fields since the doses at $10 \mathrm{~cm}$ and $20 \mathrm{~cm}$ in water observe little change when a magnetic field is present. Further, the effect of the true sensitive collection volume of the ion chamber has been found to impact the MC determined chamber response, i.e., dose to chamber's air cavity, when a magnetic field is present ${ }^{27,28}$. Air gaps surrounding the chamber have been shown to cause several percent change in the chamber response ${ }^{29-31}$. The complexity of these issues further demonstrates the need to have accurate and detailed MC simulations to bring magnetic field dosimetry in-line with established protocols.

\subsubsection{The EGSnrc simulation package}

EGSnrc $^{32}$ (Electron Gamma Shower) is a Monte Carlo simulation package which includes physical effects such as photoelectric, Compton, and pair-production interactions to produce coupled electron, position, and photon transport in user defined geometries and media. The efficient transport mechanism which uses the condensed history method, responsible for allowing large charged particle steps, and a single scatter algorithm, used for short steps on the order of the mean free path of the particle, has made EGSnrc the gold standard in the field for performing MC simulations, especially involving ion chambers. The code passes the Fano test (to be discusses below in section 3.1), considered as one of the most rigorous tests of MC transport algorithms, at the $0.1 \%$ level $^{33}$ (i.e., the simulation result produces less than $0.1 \%$ deviation from the theoretical Fano test value). A variety of true variance reduction techniques, such as photon splitting and Russian Roulette, allow for further improvements in efficiency. The code handles particle energies ranging between a few keV and several GeV. Total energy cut-off values, ECUT for electrons and PCUT for photons, are code inputs be- 
low which the respective particle simulations stop and any remaining kinetic energy is deposited locally. The selection of ECUT and PCUT, both carrying units of MeV, are chosen to improve the simulation efficiency without impacting the actual dose value. A larger cut-off value produces a faster simulation, and if particles with energy at and below the cut-off value cannot escape the dose scoring region the final result is not compromised.

Several applications of the code provide a variety of geometry packages. The DOSXYZnrc application is intended for simulating voxelized geometries, and DOSRZnrc allows the construction of cylindrical structures. The former of these applications is used for some of the depth-dose calculations in this work. The majority of simulations in this study employ egs_chamber, which is a c++ based EGSnrc application that permits the modelling of complex structures like ion chambers ${ }^{34}$.

\section{Magnetic fields in EGSnrc and other Monte Carlo codes}

Currently, PENELOPE ${ }^{35}, \mathrm{MCNP}^{36}$, and Geant4 allow for charged particle transport in magnetic fields, but EGSnrc ${ }^{37,38}$ lost this capability in the transition from EGS4. A few groups have adapted the EGS4 magnetic field macros for use in EGSnrc ${ }^{12,39}$. Essentially these changes apply the theory originally described by Bielajew ${ }^{40}$, and this one-point integration technique to determine the influence of the magnetic field was re-implemented in the January 2017 EGSnrc code release. PENELOPE makes use of a similar algorithm and recent Fano tests demonstrate differences up to approximately $1 \%$ from the expected results ${ }^{41}$. This approach requires a significant reduction in stepsize which can negatively impact the efficiency. Geant4 has also been used to calculate ion chamber doses in the presence of magnetic fields ${ }^{42}$, but there may be a significant drop in efficiency through the use of Geant $4^{43-45}$.

At the onset of the work outlined in this thesis, EGSnrc carried no explicit support 
for magnetic fields. The older implementation (in EGS4 and the EGSnrc January 2017 release) is based on a simple one point numerical integration of the Lorentz force. This approach necessitates a drastic reduction in the step size. Further, the implementation did not address the crucial task of proper boundary crossing which is necessary to allow for correct scoring of dose and to ensure that particle locations are properly registered with the system.

\subsubsection{Thesis purpose}

Many of the issues of the introduction of magnetic fields into radiation therapy are highlighted in the previous sections. These lead to the following purposes of this thesis. The first is to develop an implementation of charged particle transport in magnetic fields that is efficient and accurate. The second goal is to validate this implementation and ensure that it is sufficiently stable and reliable to be applied to ion chamber dosimetry applications. The third goal is to apply the code to magnetic field dosimetry. This last point includes addressing beam-quality specification in magnetic fields, proper orientation of the ion chamber when performing such dosimetry tasks, and the determination of magnetic field correction factors to allow ion chambers to continue to be used for clinical reference dosimetry.

\subsubsection{Thesis outline}

The thesis is structured to the reflect the progression through the purposes listed above. In chapter 1, an overview of the issues pertaining to charged particle transport and dosimetry in magnetic fields is reviewed. Chapter 2 deals with the development of the code to permit for efficient and reliable charged particle transport in magnetic fields in EGSnrc. The different transport mechanisms in EGSnrc are addressed and a specialized boundary crossing algorithm is introduced. Additionally, efficiency and stability 
algorithms are also outlined. Following this, the validation and testing of the code is performed in chapter 3. A specialized Fano test which allows for Monte Carlo transport algorithm testing in magnetic fields is implemented and applied. Comparisons to experimental ion chamber measurements are also performed. In chapter 4 the magnetic field transport code is applied to look at a simplified lung model and evaluate the importance of including lung heterogeneity in MRgRT treatment planning. Chapter 5 deals with determining the impact of the ion chamber's true sensitive volume on the simulated dose to the chamber air volume when a magnetic field is present. As noted above in Section 1.2.1, the fringing of the electric field near the stem of the chamber produces a "dead" volume region from which the central electrode cannot gather released charges, and MC simulations often omit this effect since it is fairly negligible in the absence of magnetic fields. In chapter 6 , optimal orientations of the ion chamber with respect to the magnetic field are evaluated and the issue of the sensitive volume is resolved. Having addressed many of the challenges involved in ion chamber dosimetry, in chapter 7 magnetic field correction factors are calculated for over thirty ion chamber and three parallel plate chambers, beam-quality specifiers are studied in the presence of magnetic fields, and the impact of using updated physical parameters, recommended by the ICRU-90 report, is evaluated. Concluding remarks follow in the final chapter and a few appendices serve to provide additional information on the transport mechanism. 


\section{Chapter 2}

\section{Implementing charged particle transport in magnetic fields in EGSnrc}

This chapter deals with the implementation of charged particle transport in EGSnrc. The numerical integration methods applied to the condensed history method and the analytical solutions used for the single scattering algorithm are described. The details of a boundary crossing algorithm which ensure that particles can correctly move through arbitrary geometries are given. Step size restrictions and efficiency improvement methods are addressed. These are the results of the peer-reviewed paper $I$.

\subsection{Lorentz force in a condensed history calculation}

Charged particle transport in EGSnrc is performed by grouping a large number of interactions into several steps between discrete events (creation of bremsstrahlung, knock-on electrons, etc.) which are accounted for on an individual basis. EGSnrc uses an adaptive electron/positron stepping mechanism in which larger steps are calculated using a condensed history $(\mathrm{CH})$ algorithm, PRESTA-II (default in EGSnrc), and a single scat-

tering algorithm (SS) is used for short steps ${ }^{32,33}$. In relation to the magnetic field code, the specialized case of the SS mode is given in the appropriate section below, and the PRESTA-II algorithm is outlined here. In a PRESTA-II step of length $s$, shown by the grey line in Fig. 2.1, the particle begins with an initial direction of motion, $\hat{u}(0)$, ve- 


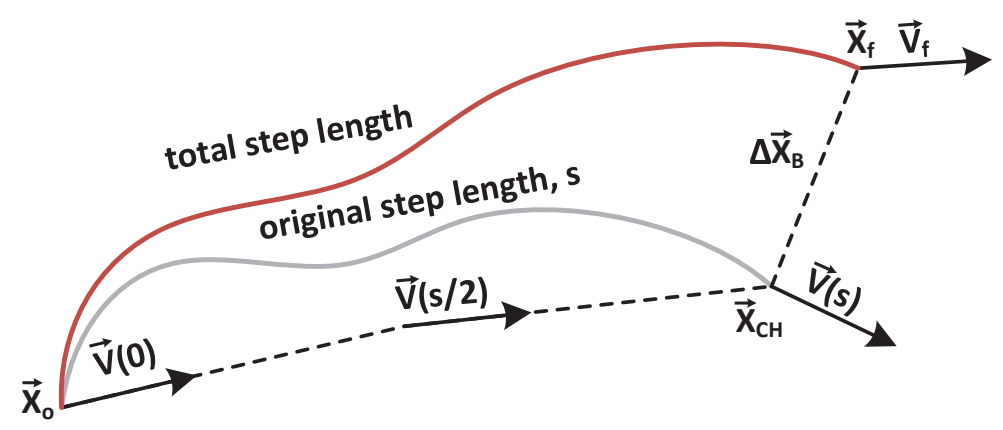

Figure 2.1: Simplified EGSnrc PRESTA-II step in the presence of a magnetic field. The particle is initially at $\vec{x}_{o}$ with velocity $\vec{v}(0)$, it is then transported a step length, $s$, to $\vec{x}_{C H}$ by the $\mathrm{CH}$ algorithm which samples direction of motion at an intermediate, $\vec{v}(s / 2)$ and final, $\vec{v}(s) . \Delta \vec{x}_{B}$ and $\Delta \vec{u}_{B}$ are calculated using Eqns. 2.2 and 2.6 to obtain the final position, $\vec{x}_{f}$, and velocity, $\vec{v}_{f}$.

locity, $\vec{v}(0)$, position, $\vec{x}_{o}$, and energy, $E_{o}$. Two scattering directions are sampled, $\hat{u}(s / 2)$ and $\hat{u}(s)$, the directions at the middle and the end of the step. These direction vectors correspond to the velocities $\vec{v}(s / 2)$ and $\vec{v}(s)$. This explanation is a slightly simplified version of the full PRESTA-II algorithm in which the first scattering angle, $\hat{u}(s / 2)$, occurs at a random distance up to $s / 2$. The full PRESTA-II algorithm is applied to all $\mathrm{CH}$ electron transport steps, and the simplification is used to facilitate the application of the three-point integration technique described below. The particle is transported to the position $X_{C H}$ by the distance given by the original step length, $s$. The energy lost is computed on the basis of $s$, and the electron moves on to the next step ( $\mathrm{SS}, \mathrm{CH}$, or a discrete event). This process is continued until the charged particle's energy falls below ECUT, at which point the particle's history is terminated and its remaining kinetic energy is deposited at its current position. To determine the influence of a magnetic field on a charged particle travelling in a material, the interplay between the medium's

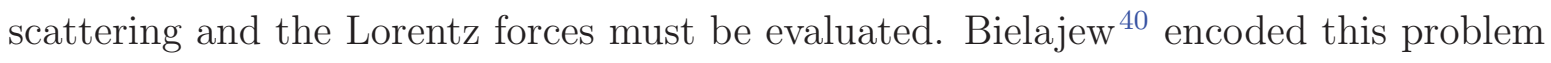
in the following general equation of motion,

$$
\frac{d \vec{p}}{d t}=\vec{F}_{m e d}(E(t))+\vec{F}_{e m}(E(t), \vec{x}(t), \vec{u}(t)),
$$


where $\vec{p}$ is the momentum, $t$ is the time, $\vec{F}_{\text {med }}$ is the force on the particle due to the medium (i.e., elastic scattering), $\vec{F}_{e m}$ is the Lorentz force due to the external electric and magnetic fields, $E(t)$ is the energy, $\vec{x}(t)$ is the particle position, and $\vec{u}(t)$ is the particle's unit direction of motion. Bielajew placed this equation in the context of a condensed history $(\mathrm{CH})$ step and demonstrated that in the case of no electric field the influence of the magnetic field on the direction of motion reduces to

$$
\Delta \vec{u}_{B}(t)=\frac{q c^{2}}{v_{o} E_{o}} \int_{0}^{t} d t^{\prime} \vec{v}\left(t^{\prime}\right) \times \vec{B}
$$

where $\Delta \vec{u}_{B}$ is the change in the direction vector due to the magnetic field during a time period $t, c$ is the speed of light, $\vec{B}$ is the magnetic field, $E_{o}$ is the particle's initial total energy, $q$ is the particle's charge, $\vec{v}(t)$ is the particle velocity, and $v_{o}=|\vec{v}(0)|$. Under the assumptions of (i) little energy loss, (ii) a constant magnetic field, and (iii) minimal change in the direction of motion during the step, a one-point numerical integration (1-PI) technique can be used to evaluate Eq. 2.2 giving,

$$
\Delta \vec{u}_{B 1}=\frac{q t c^{2}}{v_{o} E_{o}}(\vec{v}(0) \times \vec{B}),
$$

where the subscript $B 1$ indicates that this is a one-point integration for the influence of the magnetic field. The above has been used by many groups as a convenient method for determining the effect of the magnetic field in a $\mathrm{CH}$ step and complies with the assumptions made originally by Bielajew ${ }^{12,39}$. To account for energy loss and scattering during the step (i.e., weakening assumptions (i) and (iii)), the intermediate velocities in a PRESTA-II algorithm can be used to apply a three-point integration (3-PI) of Eq. 2.2 to give:

$$
\Delta \vec{u}_{B 3}=\frac{q t c^{2}}{6 v_{o} E_{o}}(\vec{v}(0)+4 \vec{v}(t / 2)+\vec{v}(t)) \times \vec{B}
$$


where the subscript $B 3$ indicates that this is a three-point integration (3-PI) for the influence of the magnetic field. Here the interaction of the magnetic field with the particle is sampled at three time positions which accommodates for the varying energy and direction of motion. $\vec{v}(t)$ is equal to $\vec{v}(s)$ in Fig. 2.1, and the velocity corresponding to $\vec{v}(s / 2)$ is used as an approximation to the velocity at $t / 2$. This assumption is used to facilitate the computation and, on average, is expected to be close enough to improve on the 1-PI technique.

To evaluate the time variable used in the numerical integration techniques, a twopoint integration of the conventional time, velocity, and distance relationship using the initial, $v_{o}$, and final, $v_{f}$, velocities can be performed. This produces the following expression for the time:

$$
t \cong \frac{s}{v_{o}}\left(\frac{1}{2}+\frac{v_{o}}{2 v_{f}}\right)=\frac{s}{v_{o}} \eta
$$

where $\eta$, the energy correction variable, is the term in the brackets. Using either integration method to obtain $\Delta \vec{u}_{B}$, the change in the position due to the magnetic field has been shown to be ${ }^{40}$

$$
\Delta \vec{x}_{B}=\frac{s \eta}{2} \Delta \vec{u}_{B}
$$

The influence of the magnetic field is added to the $\mathrm{CH}$ step as in Fig. 2.1. The position $\vec{x}_{C H}$ is adjusted by adding $\Delta \vec{x}_{B}$ to obtain $\vec{x}_{f}$. The final velocity, $\vec{v}_{f}$, is determined by adding the change in the direction of motion due to the magnetic field, calculated using Eq. 2.3 or 2.4, to the direction of $\vec{v}(t)$. To perform the addition and normalization of the direction vector properly, a procedure which adjusts the vector components perpendicular to the magnetic field while conserving the vector's magnitude in the parallel direction is used (see Appendix A for details). Overall, PRESTA-II performs a regular $\mathrm{CH}$ step and the magnetic field influence is included after the $\mathrm{CH}$ calculations.

The three assumptions made above translate into step-size restriction parameters. 
Since a constant magnetic field is being considered and there are energy loss step-size control parameters in EGSnrc already, only the restriction on the change in the direction due to the magnetic field will be considered and encoded using:

$$
\delta_{u}=\left|\Delta \vec{u}_{B}\right|
$$

recalling that $|\hat{u}|=1$, the magnitude of the direction vector, makes $\delta_{u}$ a limit on the fractional change of the direction of motion produced by the magnetic field. Given a value for $\delta_{u}$, Eq. 2.3 and 2.7 are used to obtain the step-size limit:

$$
s\left(B, \delta_{u}, E_{o}\right)=\frac{\delta_{u} \beta^{2} E_{o}}{|q \vec{v}(0) \times \vec{B}|},
$$

where $\beta$ is the particle velocity normalized by the speed of light and the $\eta$ term is set to 1 so that this step-size quantity is consistent with other implementations, in EGSnrc and PENELOPE, which use a similar magnetic field transport theory. The 1-PI equation is used here since the scattering angles and energy loss over the step are required for the 3-PI method, and this necessitates knowledge of the step-size which Eq. 2.7 is used to determine. Appendix B provides a description of an additional limit on the step length which ensures that a $\mathrm{CH}$ step does not cause the particle to improperly transition into another region due to the addition of $\Delta \vec{x}_{B}$. These restrictions represent the maximum CH step that will be permitted in the magnetic field, and discrete events, boundaries, and other step size limits related to the standard $\mathrm{CH}$ technique will also restrict certain paths.

Using a $\delta_{u}$ of 0.02 or smaller has become common for Monte Carlo simulations which use similar theories ${ }^{19,20,40,77}$, but this produces a potentially severe increase in computational time. The solution for a dose calculation in the presence of a magnetic field is expected to converge as $\delta_{u}$ decreases, and determining the largest possible $\delta_{u}$ that does not cause variations in the solution is required to improve efficiency. It is 
convenient to make use of this parameter as a benchmarking tool of the stability and efficiency of the various transport options.

The effect of the magnetic field on any ionized electrons (not including those produced through discrete interaction) along the path of the original electron or positron is neglected in both the CH and SS (below) algorithms. The justification for neglecting these ionized particles is that, due to their low energy (below ECUT), these particles should not be able to escape the region in which they are produced and the overall Lorentz force will be fairly minimal (since it is proportional to the particle velocity).

\subsection{Scaled $\delta_{u}$ algorithm}

The truncation error on a 3-PI is proportional to the fourth derivative of the function being integrated and to the fifth power of the step-size used to evaluate the integral ${ }^{46}$. Since a time independent magnetic field is simulated the truncation error is directly proportional to the magnetic field (it can be pulled out of the integral in Eq. 2.2). The error for a given step-size determined by Eq. 2.8 at a reference magnetic field, $B_{\text {ref }}$, will be halved for a calculation with $B=B_{\text {ref }} / 2$. However, by Eq. 2.8, the permitted step-size with $B=B_{\text {ref }} / 2$ in a $\mathrm{CH}$ calculation will be double that in the $B_{\text {ref }}$ case,

and the increase in the truncation error due to this larger step size will be a factor of $2^{5}$. To avoid fluctuation in truncation error at different magnetic fields, a scaling of the step-size with respect to the magnetic field is performed by using:

$$
s_{\text {scale }}\left(B, \delta_{u}, E_{o}\right)=s\left(B_{r e f}, \delta_{u}, E_{o}\right)\left(\frac{B_{r e f}}{B}\right)^{\frac{1}{5}}
$$

where $s_{\text {scale }}\left(B, \delta_{u}, E_{o}\right)$ is the scaled step-size, $s\left(B_{\text {ref }}, \delta_{u}, E_{o}\right)$ is evaluated using Eq. 2.8, and $B$ is the magnetic field that the step-size is being sought at. Scaling with the fifth root is selected to account for the possibility of the error not being directly proportional 
to the magnetic field. Applying a fourth or sixth power scaling causes only about a $4 \%$ difference from the scaled step-size calculated by Eq. 2.9, which is relatively small compared to a doubling of the step-size if no scaling is used. The reference magnetic field and $\delta_{u}$ values are chosen based on Fano test calculations which produce accurate results. The chosen $B_{\text {ref }}$ is set to $1.5 \mathrm{~T}$. When this option is turned on, $s_{\text {scale }}\left(B, \delta_{u}, E_{o}\right)$ replaces the $\mathrm{CH}$ mode step-size restriction given in Eq. 2.8, and effectively attempts to maintain the error of the numerical method constant regardless of the strength of the B-field.

\subsection{Adaptive integration algorithm}

A downside of using 3-PI is a marked increase of the computational time. An improvement in efficiency can be achieved by using an algorithm which utilises 1-PI and 3-PI methods, depending on the step-size. The "Adaptive Integration" algorithm uses 1-PI whenever the step-size from other constraints is $\leq s_{\min }$, evaluated with a $\delta_{u}$ of 0.05 , since this is faster and accurate for small steps. Above $s_{\text {min }}$, the 3-PI is used to achieve better accuracy and $s$ is capped at $s_{\max }$, evaluated with a $\delta_{u}$ of 0.2 . The values for the lower and upper $\delta_{u}$ values are chosen on the basis of Fano test results found in chapter 3. A range of lower $\delta_{u}$ values for establishing $s_{\min }$ were tested to ensure passing of the Fano test and to balance efficiency.

\subsection{Magnetic field influence in single scatter (SS) mode}

The SS mode in EGSnrc was originally introduced to deal with boundary transitions and to improve the accuracy of small steps. In a regular SS step, without a magnetic field, the particle travels linearly in its initial direction of motion and, assuming it doesn't reach a boundary, it has an elastic scattering event at its final position to determine the 
resulting direction. In a magnetic field, an analytical expression for charged particle transport in a magnetic field in vacuum ${ }^{40}$ is used instead of applying an approximation to the transport by using Eqs. 2.2 and 2.6, and the linear transport of the particle is bypassed. One of the main assumptions of applying the analytical solution is that there is little energy loss over each of the SS steps (Appendix C verifies the validity of this assumption).This method facilitates boundary crossing, and ensures nearly exact transport for the SS step.

\subsection{Boundary crossing}

Without a magnetic field, boundary crossing in EGSnrc is performed using linear steps in the SS mode, the default boundary crossing algorithm in EGSnrc. When a stepsize, $s$, larger than the shortest perpendicular distance to the nearest boundary, $t_{\text {perp }}$, is to be taken within a distance called skin-depth (set to three elastic mean free paths by default) to a boundary, a howfar call is made to determine the distance to the boundary in the initial direction of motion, $d_{H F}$. If $d_{H F}$ is smaller than $s$ the step is shortened and the boundary is crossed using linear transport. In the presence of a magnetic field, the particle's trajectory will curve and the final transport may leave the particle in the current region or across the boundary. This can cause incorrect region transitions and can lead to erroneous scoring. To properly transition the particle in a magnetic field, a specialized boundary crossing algorithm (BCA) is introduced, as shown in Fig. 2.2. This algorithm applies only to the SS mode, and the PRESTA-I BCA option of EGSnrc does not support magnetic fields at this time. When a step-size to the next scatter, $s_{o}$, is larger than $t_{\text {perp }}$ and within the skin-depth to a boundary,

the particle is transported a distance $t_{\text {perp }}$ using the analytical expression for charged particle transport in a magnetic field in vacuum instead of performing a check using howfar. At the end of this step a new perpendicular distance to nearest boundary, 
$t_{p e r p 2}$, is evaluated and the particle is transported further by this distance, making the total step length to this point $s_{2}=t_{\text {perp }}+t_{\text {perp } 2}$. The process of recalculating $t_{\text {perp }}$ continues until it is within a specified cut-off distance to a boundary (encoded by the \$BCA_lin_buffer macro with a default value of $10 \mathrm{~nm}$ ) is reached or the allowed total step length is found to be larger than $s_{o}$, to which the step-size is capped. Here a small linear step is then taken to transition into the next region, but the final direction of motion is determined using the analytical expression. In the case when a region transition is not required, $\Delta \vec{x}_{B}$ is applied to the position when $\left|\Delta \vec{x}_{B}\right|<t_{\text {perp }}$ at the end of the linear transport. Different \$BCA_lin_buffer values were tested using ion chamber dose calculations. No effect on dose calculations is found for values below $1 \times 10^{4} \mathrm{~nm}$. The conservative value of $10 \mathrm{~nm}$ is chosen to ensure accuracy since there is little effect on the timing.

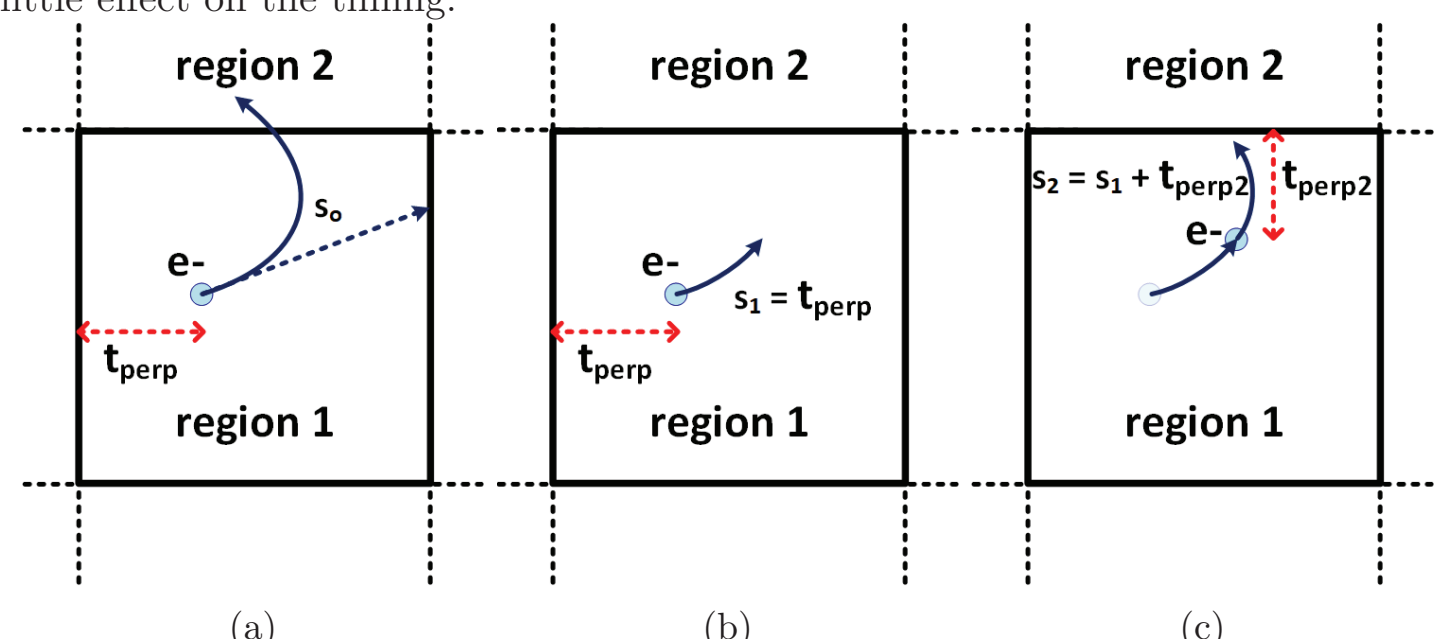

Figure 2.2: Single scatter boundary crossing in the presence of magnetic fields. (a) An intended step larger than $t_{p e r p}$ is to be taken. Linear (dashed) and magnetic field (solid) transport is shown. (b) The step-size is shortened to $t_{\text {perp }}$ and the particle is transported using analytical solutions for magnetic field transport in vacuum. (c) A new $t_{\text {perp }}$ is calculated and used to transport the particle further by this distance. 


\subsection{Summary}

The implemented magnetic field transport code accounts for the impact of the Lorentz force through numerical integration for the $\mathrm{CH}$ algorithm and an analytical solution for the SS transport. The use of a three-point numerical integration technique is intended to improve the reliability of the numerical solution, while the one-point integration method is still applied for small step sizes to reduce the impact on the code efficiency, as studied in the following chapter. The analytical solution used in the single scattering algorithms allows for efficient transport and permits the use of the specialized boundary crossing technique. Overall, the implemented code can be easily ported into any EGSnrc application and is able to accommodate any properly implemented EGSnrc geometry package. 


\section{Chapter 3}

\section{Validation of magnetic field transport code}

In this chapter, the magnetic field transport code is tested using a Fano test, adjusted for the presence of the magnetic field, and experimental results of normalized ionization chamber readings as a function of magnetic field. Further the ion chamber geometry is used to evaluate the impact of the code on the timing and efficiency of the simulations. These are the results of the peer-reviewed paper $I$.

\subsection{Fano cavity test}

The Fano theorem has been applied to produce a strict test of Monte Carlo algorithms (testing against the code's own cross sections and transport mechanisms). It states that as long as cross-sections are independent of density and charged particle equilibrium exists, the electron spectrum is also independent of density ${ }^{47}$. Given the electron spectrum, the dose to various region of the geometry can then be predicted and a $\mathrm{MC}$ code can be tested against these quantities. To perform the test for a Monte Carlo code, a cavity region with a density 1000 times lower than the surrounding material ${ }^{33}$ is used. In such tests, without a magnetic field, EGSnrc passes at the $0.1 \%$ level $^{33}$. An alternative implementation of the Fano test was introduced by Sempau and Andreo ${ }^{48}$ in which the photon beam is circumvented and a uniform intensity per unit mass source of electrons is simulated in two semi-infinite slabs of dense material with a gap of low density 
material in between. By using the reciprocity theorem, a thin source of electrons can be employed and the dose scored in the entire gas region. This test demonstrated that, with careful selection of transport parameters, the PENELOPE code passes the Fano test near the $0.1 \%$ level with deviations as high as $0.4 \%$ for certain configurations ${ }^{48}$.

Bouchard and Bielajew ${ }^{49}$ have demonstrated that the assumptions of the Fano cavity theorem are broken when a magnetic field is introduced. Bouchard et al. ${ }^{50}$ introduced two alternative modifications to the Fano cavity test, either of which make it valid in magnetic fields. The two alternatives are: (1) Using an isotropic uniform per unit mass source of electrons and (2) applying a magnetic field that scales with the mass density of the material. Pooter et al. implemented the first variant of the Fano cavity test in the PENELOPE code and observed deviations from the expected results near $1 \%$ for a $1.5 \mathrm{~T}$ magnetic field ${ }^{41}$ for $4 \mathrm{MeV}$ initial electron energy.
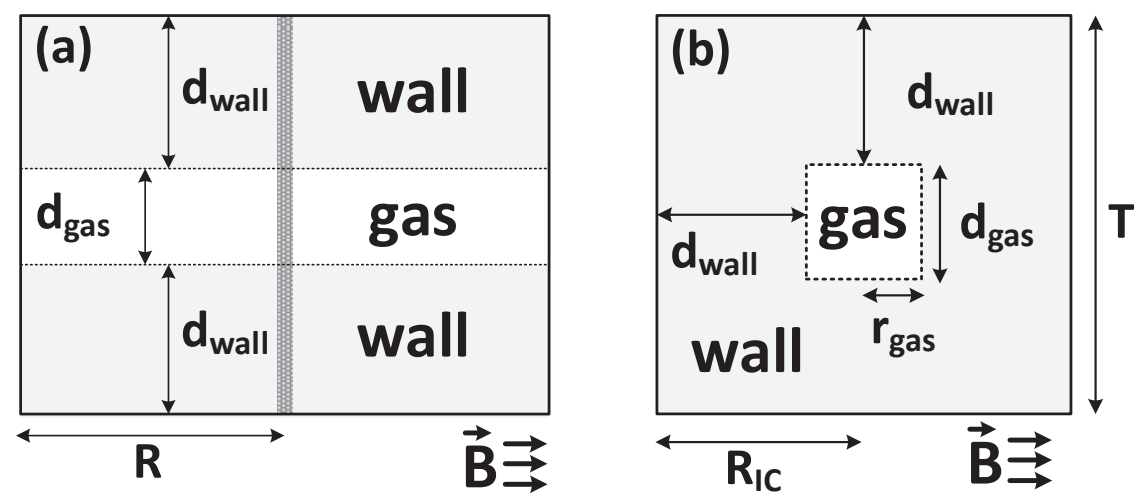

Figure 3.1: (a) Cross-section through the slab Fano test geometry. The shaded region in the centre corresponds to the isotropic uniform per unit mass electron source. (b) Cross-section through the cylindrical ion chamber (IC) Fano test geometry. Electrons are generated isotropically on a per unit mass basis throughout the geometry.

A test of magnetic field transport algorithm is implemented in the EGSnrc DOSRZnrc application using the Sempau and Andreo Fano test approach. Two cylindrical geometries are simulated. In the first geometry (slab Fano test), a low density gas slab of thickness $d_{\text {gas }}$ is placed between two slabs of the wall material each with thickness $d_{\text {wall }}$ (see Fig. 3.1a). The entire cylinder has a radius $R$ equal to 1.4 times the CSDA 
range, $R_{C S D A}$, of the initial kinetic energy of the electrons in the gas. A thin, isotropic, uniform per unit mass electron source is simulated at the central axis. In the second geometry (ion chamber Fano test), Fig. 3.1b, a vertical gas cylinder of radius $r_{\text {gas }}$, set to $0.35 \mathrm{~cm}$, and thickness $d_{\text {gas }}$ is surrounded by the wall material having a total thickness $T$ and radius $R_{I C}$, such that the distance between the gas region and the outer edges of the wall cylinder is at least $d_{\text {wall }}$. An isotropic uniform per unit mass electron source is simulated everywhere in the ion chamber Fano test geometry. In both geometries $d_{\text {wall }}$ is set to be 1.4 times the $R_{C S D A}$ of the initial kinetic energy of the electrons in the wall. Gas thickness, $d_{\text {gas }}$, is simulated as 0.2 or $2 \mathrm{~cm}$ for the slab Fano test geometry, and $2 \mathrm{~cm}$ in the ion chamber Fano test. Graphite is used as the simulation material, with the wall having a density of $1.7 \mathrm{~g} \mathrm{~cm}^{-3}$. The gas density is 1000 times less but with the same cross-sections including the density effect as the wall. A magnetic field perpendicular to the central axis of the cylinders is simulated in both geometries. Electron kinetic energies of $0.01,0.1,1$, and $10 \mathrm{MeV}$ are simulated. Statistical uncertainties for the $10 \mathrm{MeV}$ cases are below $0.09 \%$ and below $0.03 \%$ for all other energies. As required by this version of the Fano test, all photons produced in the simulation are terminated and their energy is deposited locally. The electron total energy threshold is set to $512 \mathrm{keV}$ and secondary electrons are tracked as well. Dose, $D$, is scored in the gas regions of both geometries, and the ratio, $Q$, of the $\mathrm{MC}$ dose to the expected result, $I E_{o}$, is defined as:

$$
Q=\frac{D}{I E_{o}}
$$

where $I$ is the number of initial electrons per unit mass, and $E_{o}$ is the initial kinetic energy of the source electrons. In the ideal case, $Q$ would be equal to 1 . For the slab Fano test geometry the $I$ variable in Eq. 3.1 is substituted with:

$$
I_{\text {slab }}=\frac{N}{R^{2} \pi\left(2 d_{\text {wall }} \rho_{\text {wall }}+d_{\text {gas }} \rho_{\text {gas }}\right)}
$$


where $\rho_{\text {gas }}$ and $\rho_{\text {wall }}$ are the gas and wall densities, and $N$ is the number of particle histories used for the calculation. For the ion chamber Fano test geometry the $I$ variable in Eq. 3.1 is:

$$
I_{I C}=\frac{N}{\rho_{\text {wall }} \pi\left(T R_{I C}^{2}-d_{\text {gas }} r_{\text {gas }}^{2}\right)+\rho_{\text {gas }} d_{\text {gas }} r_{\text {gas }}^{2} \pi} .
$$

The slab Fano test is verified by calculating the degree of agreement EGSnrc has with the theoretical Fano value for the NULL field case. The ESTEPE variable, which sets the maximum fractional energy loss over a single CH or SS step, is varied from 0.01 to 0.25 to determine any step-size effects. To evaluate the effect of the magnetic field code, on both integration methods, a magnetic field ranging in magnitude from 0 to $5 \mathrm{~T}$ for $\delta_{u}$ values of 0.02 and 0.2 is simulated for the slab Fano test. Additionally, a study as a function of $\delta_{u}$ is performed for the $1.5 \mathrm{~T}$ case for both the slab and ion chamber Fano tests. The magnetic field is simulated perpendicular to the radial axis of the geometries as it was found that simulations with the parallel case pass the test more easily.

\subsubsection{Results and discussion}

Fig. 3.2 shows the percent deviation of the MC calculated Fano dose from the theoretical value for the NULL field case as a function of ESTEPE for the $0.2 \mathrm{~cm}$ and $2 \mathrm{~cm}$ thick gas region. These simulations are run with the default XIMAX value and with isotropically radiating electrons from the source region. In all cases, deviations fall below the $0.1 \%$ threshold. The ESTEPE $=0.25$ case corresponds to the default EGSnrc setting, i.e. no tuning of transport parameters is needed to pass. These results are in agreement with Fano tests performed with ion chambers in photon beams ${ }^{38,51}$. 


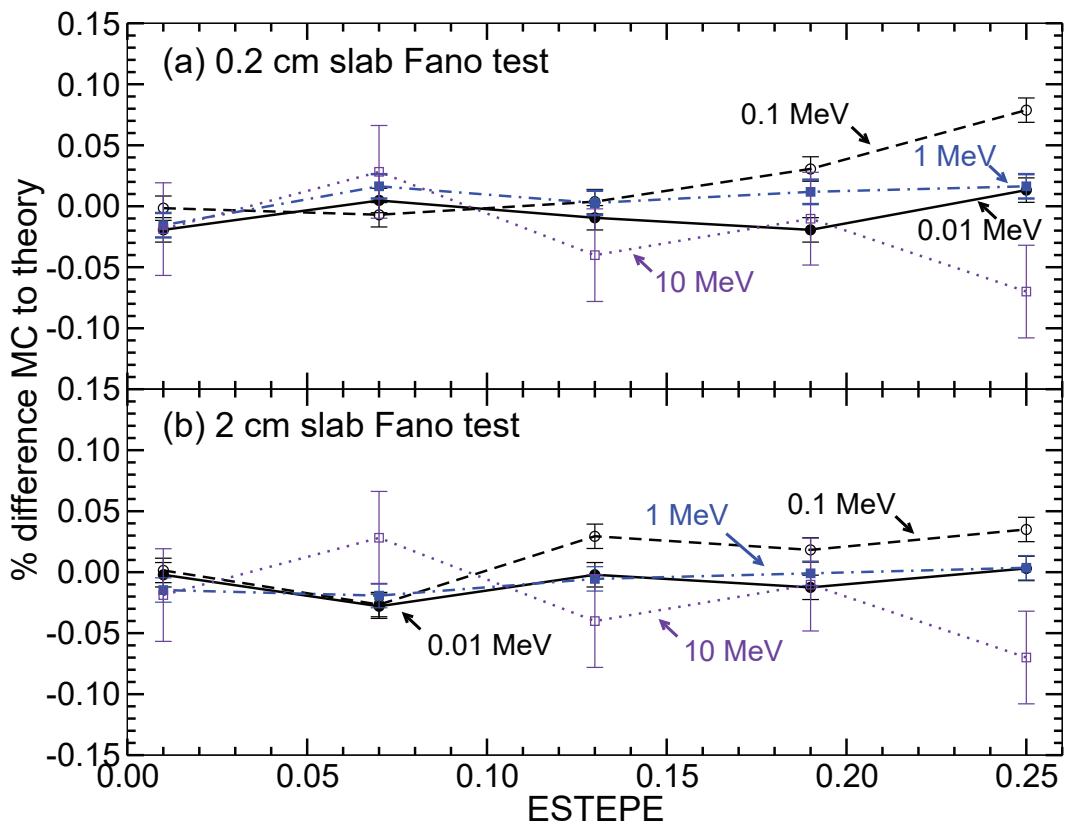

Figure 3.2: Percent deviation of the Monte Carlo calculated Fano test dose from the theoretical value for the no magnetic field case as a function of the ESTEPE parameter for (a) $0.2 \mathrm{~cm}$ gas gap and (b) $2 \mathrm{~cm}$ gas gap .

With the magnetic field on, Fig. 3.3 provides the percent deviation from the theoretical Fano test result for the $0.2 \mathrm{~cm}$ gas gap thickness for the 1-PI and 3-PI methods. At low $\delta_{u}$ values, for both integration techniques the results are within the $0.1 \%$ range, however large deviations are seen in the 1-PI calculations at higher $\delta_{u}$ values. The 3-PI provides improved stability even to $\delta_{u}$ values as high as 0.4. Fig. 3.4 is the same study but for a $2 \mathrm{~cm}$ gas gap thickness. Again, agreement on the order of $0.1 \%$ is seen at a $\delta_{u}$ of 0.02 , but the 1-PI results deviate from the expected results for the higher $\delta_{u}$ values. Variations in the 3-PI results are observed for $\delta_{u}$ larger than 0.2 , however the differences are bounded by $0.25 \%$ for the $10 \mathrm{MeV}$ case and $0.1 \%$ for the lower energies. The step-size dependence observed in the $2 \mathrm{~cm}$ case can be attributed to an increased number of particles having to be tracked to the outer radius of the Fano test geometry in the gas. 


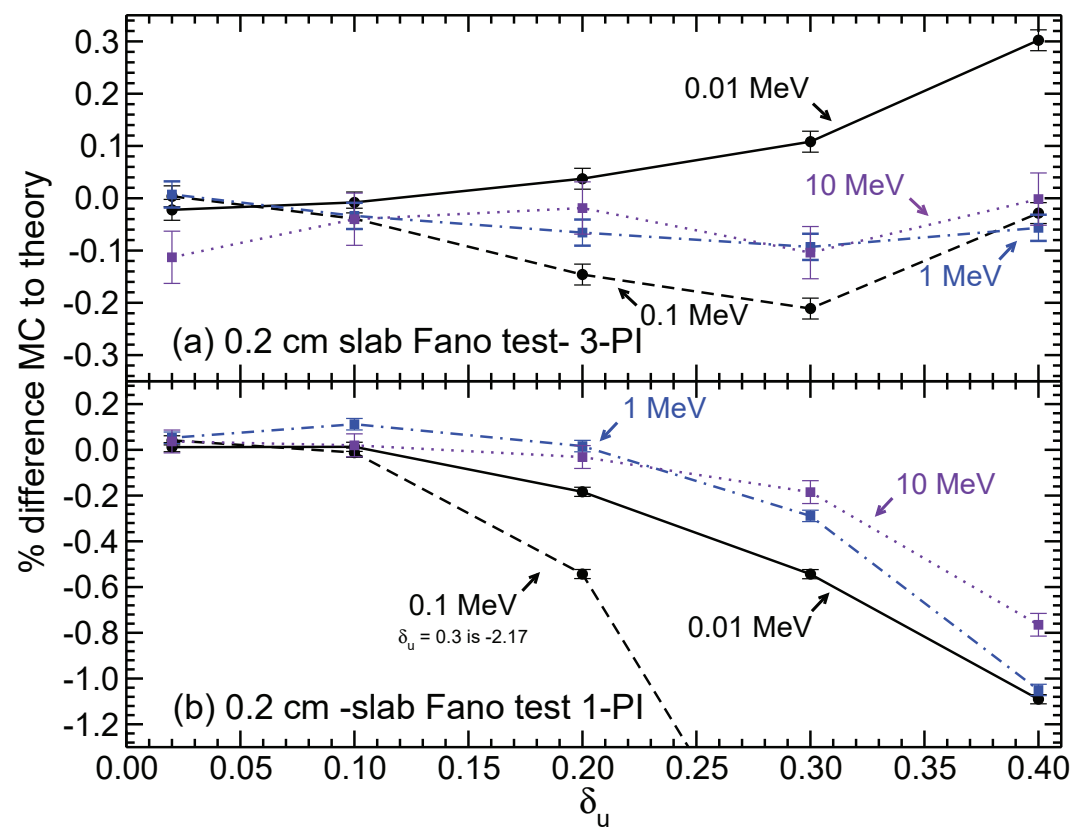

Figure 3.3: Percent deviation of the Monte Carlo calculated Fano test dose from the theoretical value for the case of a uniform 1.5 T magnetic field parallel to the wall-gas interface with a $0.2 \mathrm{~cm}$ gas gap thickness. The (a) 3-PI and (b) 1-PI results are shown.

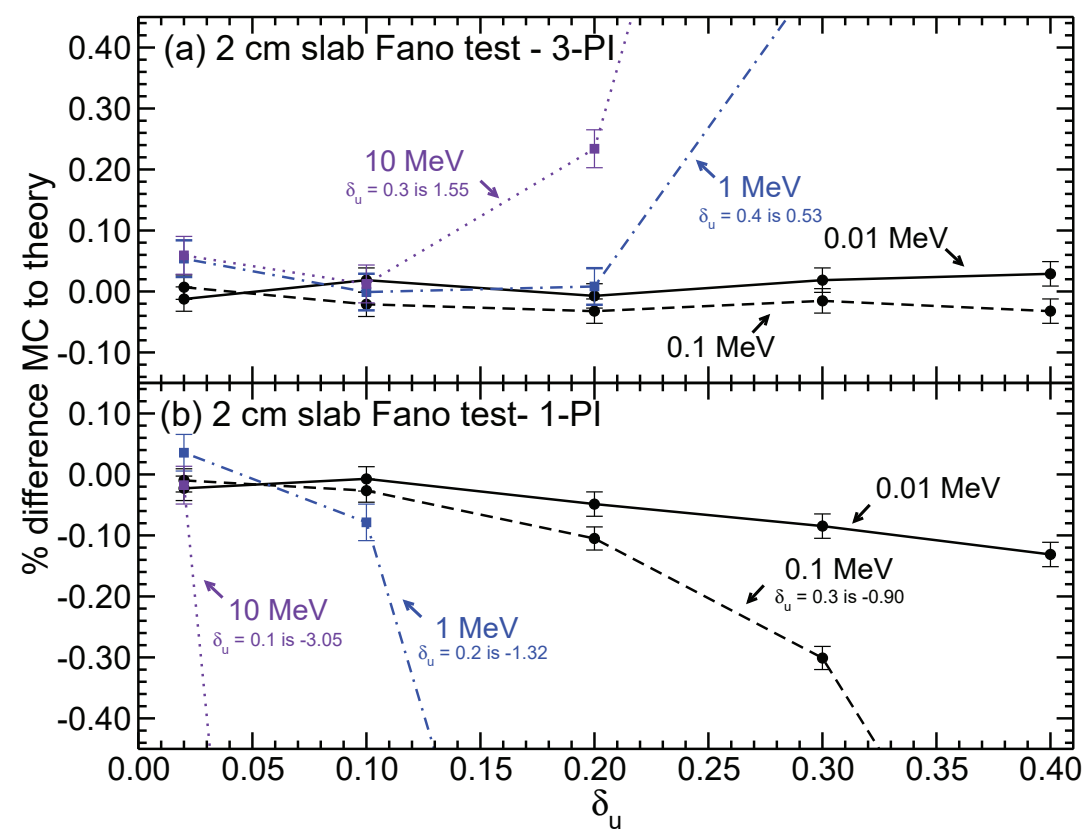

Figure 3.4: Same as Fig. 3.3 but with a $2 \mathrm{~cm}$ gas gap.

The results for the ion chamber (IC) Fano test are given in Fig. 3.5. The 3-PI results fall within $0.1 \%$ deviations from the theoretical value, while the 1-PI calculations do not exceed differences beyond $0.4 \%$. These results are much better than the slab Fano 
test results and demonstrate that not all Fano test geometries are as rigorous a test of magnetic field Monte Carlo codes.

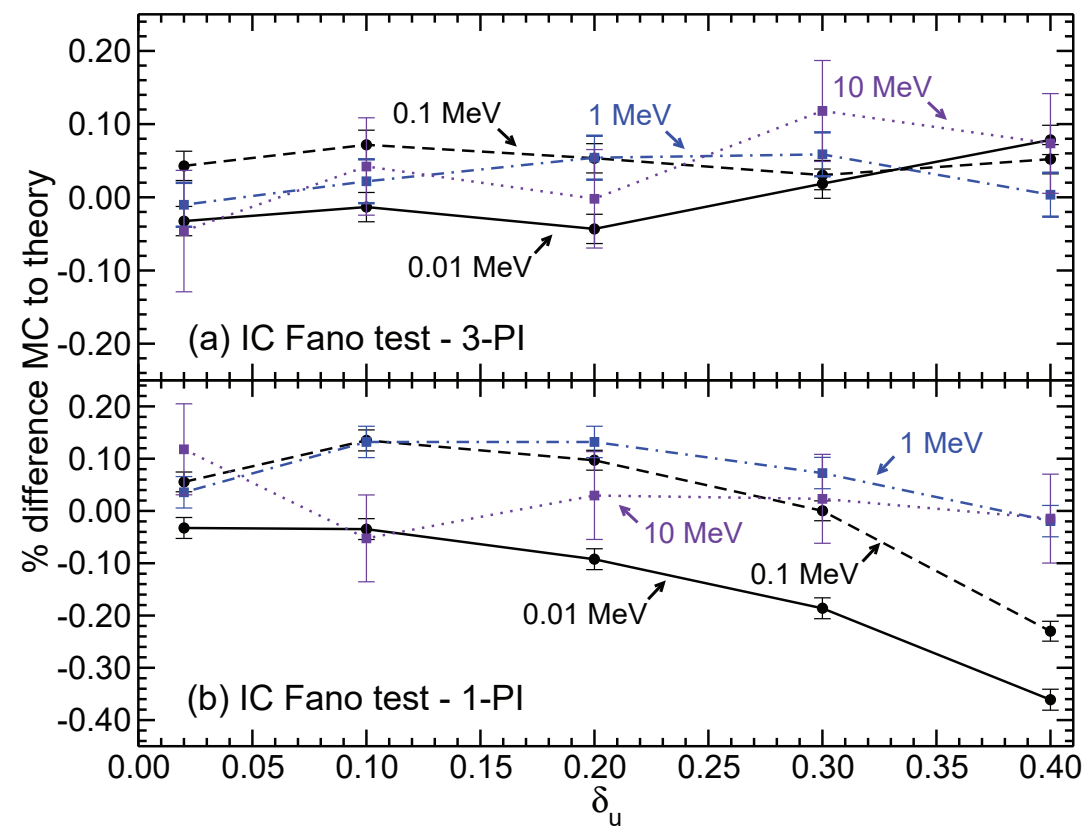

Figure 3.5: Percent deviation of the Monte Carlo calculated IC Fano test dose from the theoretical value for a $0.35 \mathrm{~cm}$ radius and $2 \mathrm{~cm}$ thick gas region. The (a) 3-PI and (b) 1-PI results are shown.

Fig. 3.6 demonstrates the dependence of the Fano test result on the magnitude of the magnetic field for the 0.2 and $2 \mathrm{~cm}$ gaps for a $1 \mathrm{MeV}$ isotropic electron source. The 1-PI results in large deviations throughout the magnetic field range for a $\delta_{u}$ value of 0.2 . An increase in the difference is seen for the 3-PI results at lower magnetic field values for a $\delta_{u}$ of 0.2 . A $\delta_{u}$ of 0.1 eliminates the deviations. The variations at the higher $\delta_{u}$ are related to the improper scaling of the step-size restriction as a function of magnetic field. Applying the "Scaled $\delta_{u}$ " algorithm with $1.5 \mathrm{~T}$ as the reference magnetic field and using a $\delta_{u}$ of 0.2 results in very good agreement with the Fano test. Turning on the "Scaled $\delta_{u}$ " with or without the "Adaptive Integration" algorithms gives agreement within $0.1 \%$, as seen in in Fig. 3.6. 


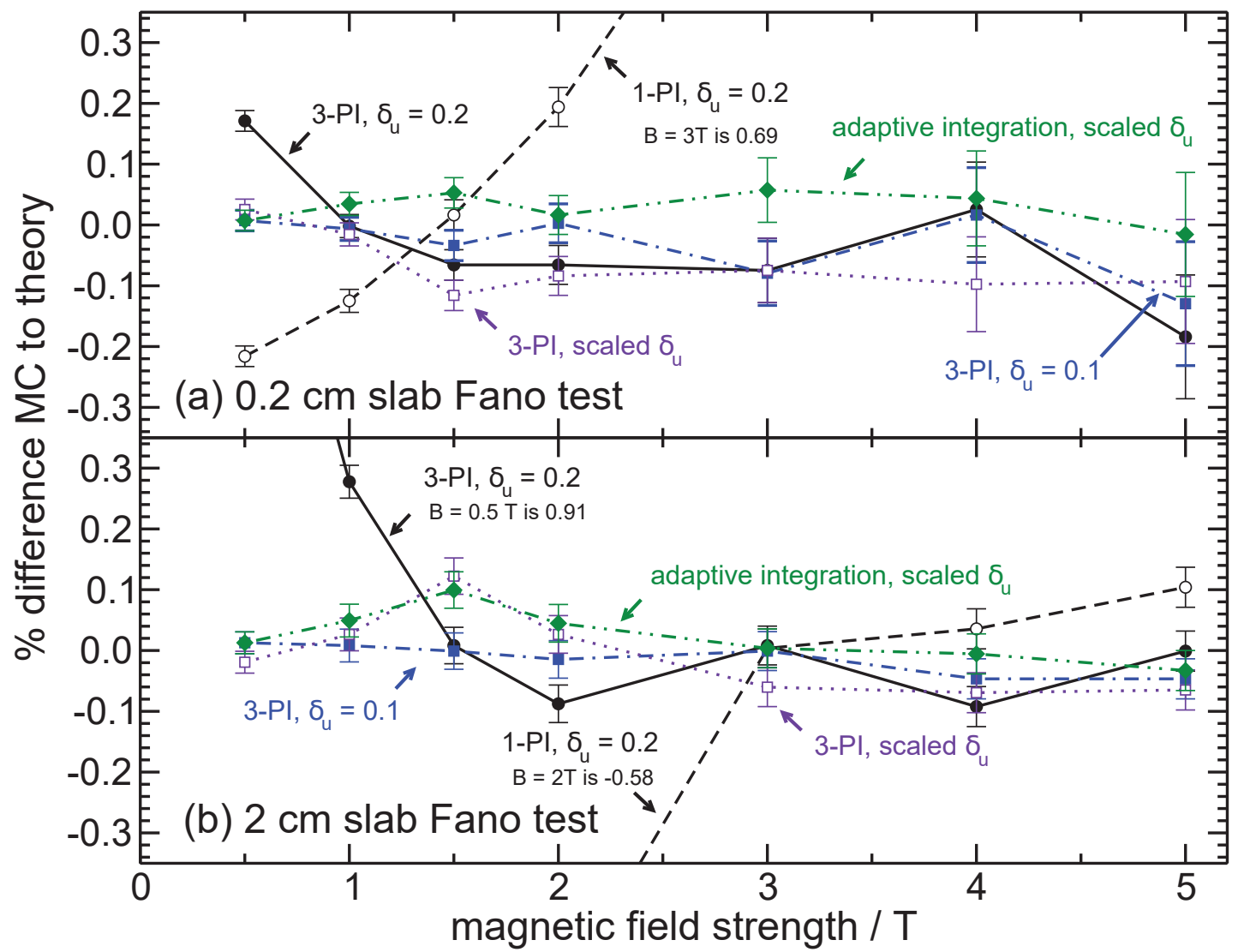

Figure 3.6: Percent deviation of the Monte Carlo calculated Fano test dose from the theoretical value as a function of magnetic field for the (a) $0.2 \mathrm{~cm}$ and (b) $2 \mathrm{~cm}$ thick gas region with a $1 \mathrm{MeV}$ isotropic electron source.

\subsection{Comparison to experimental ion chamber measurements}

To further benchmark the magnetic field transport code, the dose as a function of magnetic field, normalized to the $0 \mathrm{~T}$ case, to the sensitive volume of the NE 2571 ion chamber is determined and compared to the experimental results of Meijsing et $a l^{42}$.

All ion chamber simulations were performed using the EGSnrc Monte Carlo system with the egs_chamber ${ }^{52}$ application. Variance reduction techniques are used to conserve CPU time and all default parameters are maintained for the simulations with the exception of using the NIST bremsstrahlung cross-sections. The photon and electron total energy thresholds were set to $10 \mathrm{keV}$ and $521 \mathrm{keV}$, respectively. 
To be consistent with the measurement set up of Meijsing et al ${ }^{42}$ who used 2 ion chamber configurations, calculations were performed in a delrin cylinder with a length of $6.9 \mathrm{~cm}$ and diameter of $4.3 \mathrm{~cm}$. A parallel $6 \mathrm{MV}$ photon beam ${ }^{42}$ (Elekta SLi25 spectrum ${ }^{53}$ ) incoming from the negative z-direction and a uniform magnetic field in the $\mathrm{y}$-direction is simulated throughout the phantom using the EGSnrc magnetic field package implemented in this work. Calculations, not presented here, with a 6 MV Varian linac spectrum revealed no sensitivity of the final results to the choice of spectrum for the same energy. In the first configuration, CI, the ion chamber has its central axis perpendicular to both the magnetic field and the photon beam, i.e. chamber's central axis lies in the $\mathrm{x}$-direction. In the second configuration, CII, the ion chamber remains perpendicular to the magnetic field but is now parallel to the photon beam i.e., the long axis of the chamber is in the z-direction. These geometrical configurations are shown in Fig. 3.7. The NE 2571 ion chamber model used for these simulations has been previously described by La Russa et $a l^{54}$. With omission here of the stem geometry description, the chamber consists of an active air volume with a length of $24.0 \mathrm{~mm}$ and a radius of $3.14 \mathrm{~mm}$, a solid aluminium electrode with a length of $20.5 \mathrm{~mm}$ and a radius of $0.5 \mathrm{~mm}$, and a graphite wall with a thickness of $0.36 \mathrm{~mm}$. The conical tip on the end of the chamber is also simulated. 


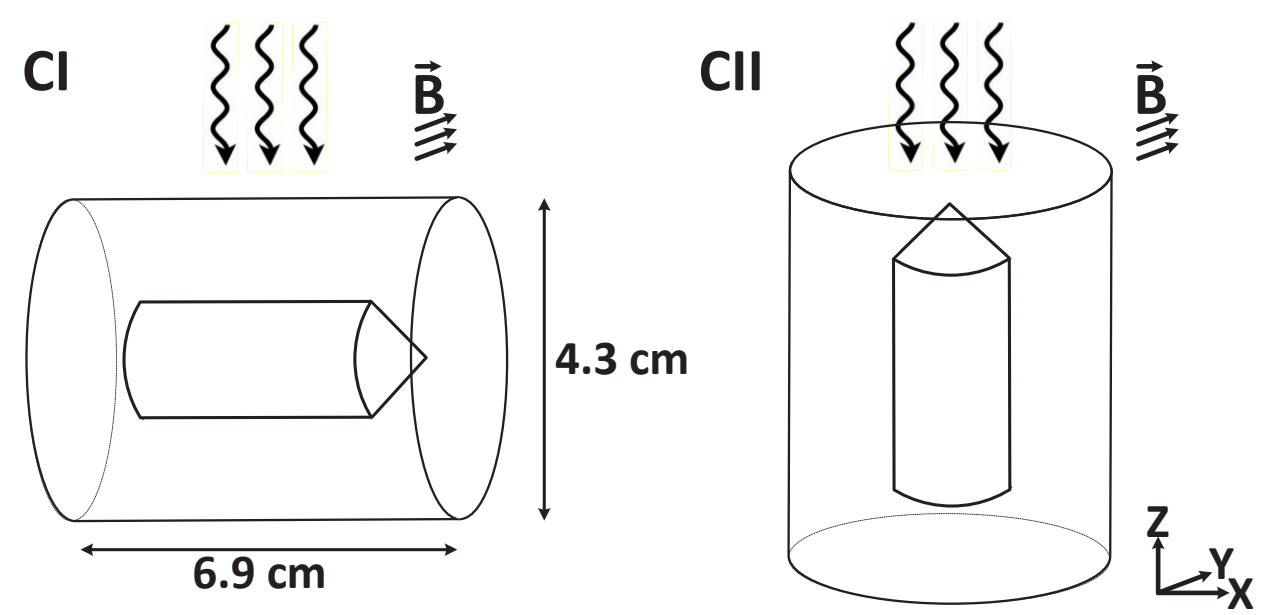

Figure 3.7: Geometrical set up of ion chamber simulations with an incoming parallel $6 \mathrm{MV}$ photon beam for configurations I and II (CI and CII). In both configurations the magnetic field is in the y-direction. A simplified ion chamber model is shown with the surrounding delrin build-up cap.

The dose to the active volume of the chamber is scored as a function of magnetic field strength, ranging from 0 to $2 \mathrm{~T}$ in increments of $0.15 \mathrm{~T}$. The dose is normalized by the dose obtained in the same geometry but in the absence of the magnetic field. Note that the normalization is performed using the original (no magnetic fields) EGSnrc simulation package, which is defined as the NULL EGSnrc case. All dose values are calculated to below $0.02 \%$ statistical uncertainty. The values for both CI and CII configurations are compared to the experimental results obtained by Meijsing et al ${ }^{42}$. Testing is performed for the 1-PI and 3-PI with the new BCA and specialized SS mode turned on.

\subsubsection{Results and discussion}

Figs. 3.8 and 3.9 provide the NE 2571 cavity doses in magnetic fields in the range of 0 to $2 \mathrm{~T}$ for configurations I and II normalized by the NULL EGsnrc cavity dose. Unless the specific algorithm is indicated, the calculations are performed with the "Adaptive Integration" and "Scaled $\delta_{u}$ " options turned on ("Full algorithm"). The experimental data, marked with diamonds, are from Meijsing I. et al. ${ }^{42}$. At a $\delta_{u}$ of 0.02 the two 
integration techniques agree with each other. The 3-PI does not change with a $\delta_{u}=0.2$, which does not hold true for the 1-PI. These findings are consistent with the Fano test results, where the 1-PI was determined to be unstable at higher $\delta_{u}$ values. From this we determine that a $\delta_{u}$ of 0.2 is sufficiently stable for the 3-PI and use these setting for all subsequent ion chamber calculations.

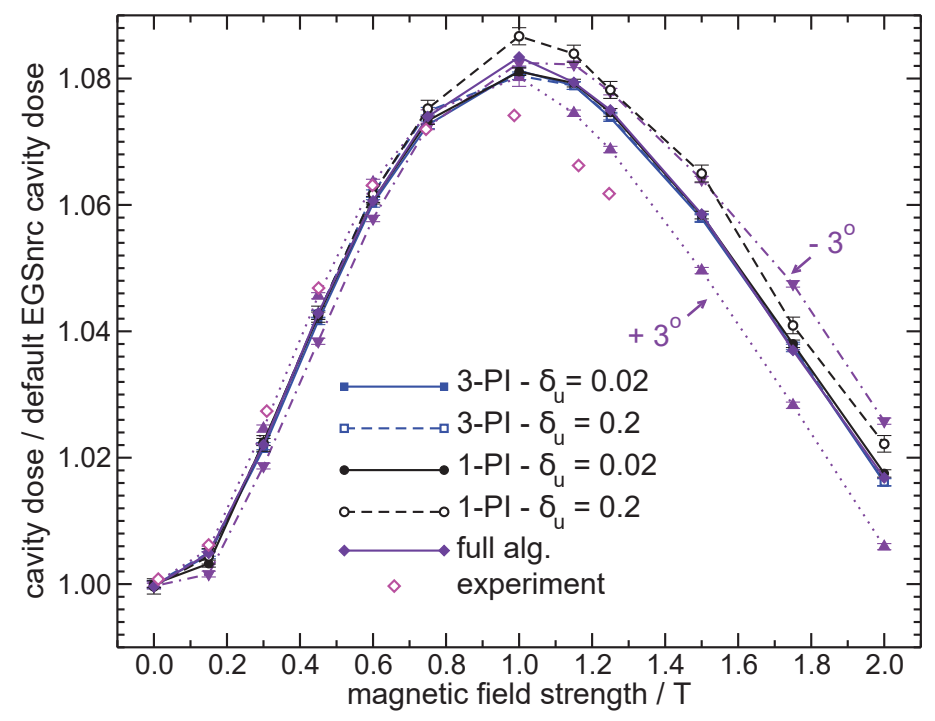

Figure 3.8: Configuration I (see Fig. 3.7) NE 2571 cavity dose as a function of magnetic field strength normalized by the NULL EGSnrc cavity dose. Experimental data are from Meijsing et al. ${ }^{42}$.

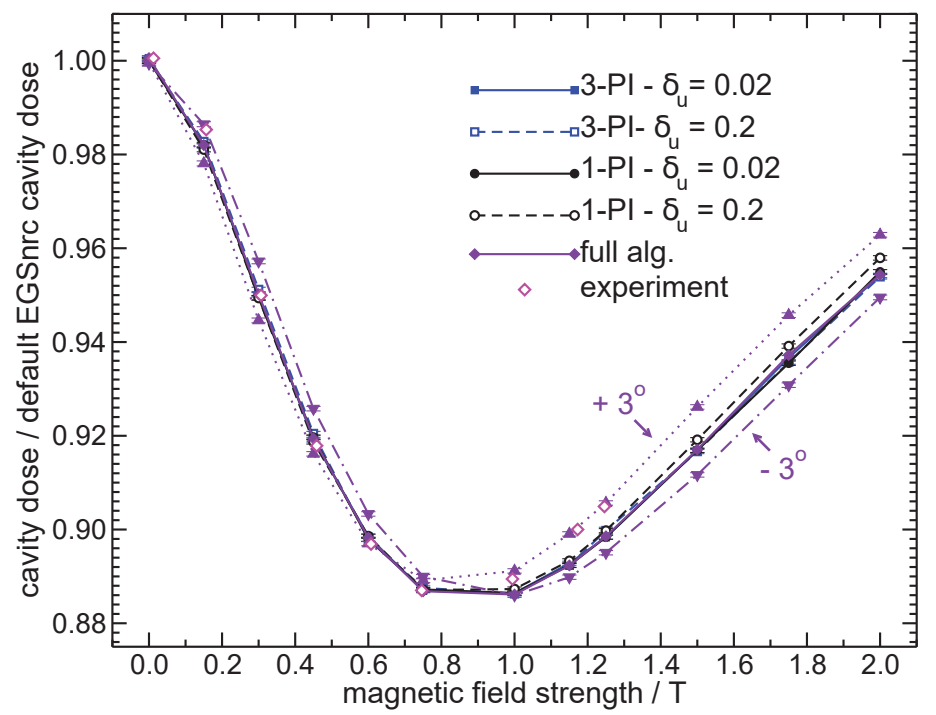

Figure 3.9: Same as Fig. 3.8 but for configuration II.

Reasonable agreement with experiment is seen up to magnetic field values of $1.0 \mathrm{~T}$, at which point a slight deviation is observed. Meijsing et al. ${ }^{42}$ proposed that a tilt in 
the incoming beam with respect to the magnetic field lines may have caused a change in the ion chamber doses, but the authors could not verify if a tilt was present. We confirm that a $3^{\circ}$ variation in the incoming beam results in deviations of the normalized dose curve from the $0^{\circ}$ case, and find that a $+3^{\circ}$ degree tilt produces improved agreement with the experiment for both CI and CII geometries. Further, small variations, within a few degrees, in the alignment of the ion chamber axis with respect to the photon beam does not produce changes in the dose response for a given magnetic field.

An additional unknown in the experimental set up is the possible presence of air gaps around the graphite walls of the chamber ${ }^{55}$. High density media dampen the effect of the deflection caused by the magnetic field, and a substitution with a low density medium like air can have a noticeable effect on the electron trajectories. The normalized ion chamber doses are calculated for a uniform 0.5 or $1.0 \mathrm{~mm}$ air gap around the walls of the ion chamber, including the conical tip, and are presented in figures 3.10 and 3.11 where each of the cavity dose curves as a function of magnetic field are normalized by the NULL EGSnrc simulation (no B field) with the corresponding air gap around the chamber. In the NULL case the introduction of a $1.0 \mathrm{~mm}$ air gap causes a $0.2 \%$ increase for CI and less than a $0.1 \%$ increase for CII. Variations on the order $1.0 \%$ or lower are seen for both air gap sizes in the magnetic field cases, with the $1.0 \mathrm{~mm}$ gap producing a more pronounced effect. For CI, at magnetic fields below $1.0 \mathrm{~T}$ the air gaps produce an increase in the chamber response. In the range of approximately 0.9 to $1.0 \mathrm{~T}$ the effect of the air gap decreases and a reduction in the dose is observed for higher magnetic fields. This situation is reversed for CII, where an initial reduction in the dose is seen with a subsequent increase. These effects are a product of a balance of electrons being swept into or out of the chamber's sensitive volume as a function of magnetic field. 


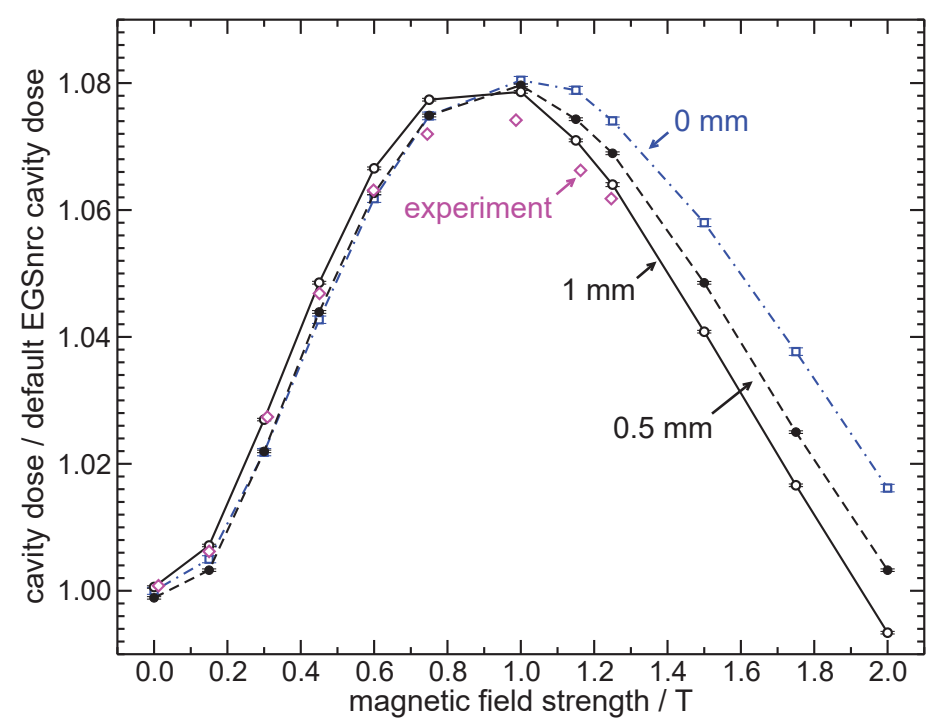

Figure 3.10: Configuration I NE 2571 cavity dose with air gaps around the wall of the chamber as a function of magnetic field strength normalized by the NULL EGSnrc.

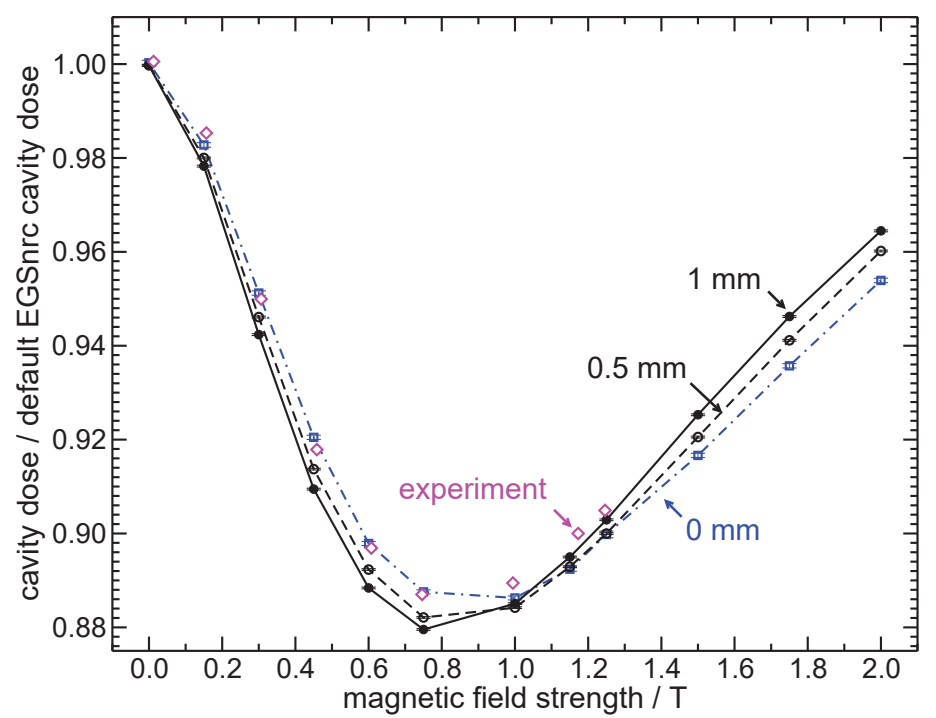

Figure 3.11: Same as Fig. 3.10 but for configuration II.

The complexity introduced by the magnetic field accentuates experimental details that might otherwise appear to be inconsequential. Very detailed experimental descriptions are required to provide corresponding accurate Monte Carlo results, and as is shown in Chapter 5, the sensitive volume of the ion chamber is an important component of the MC simulation that can drastically alter the calculated chamber dose when a magnetic field is present. 


\subsection{Impact on timing and efficiency}

Configuration I from Section 3.2 is used to determine the impact of the magnetic field code on the simulation efficiency and timing. The normalized efficiency $\left(\varepsilon_{\text {norm }}=\varepsilon_{o} / \varepsilon_{B}\right)$ and normalized time per history $\left(t_{\text {norm }}=t_{B} / t_{o}\right)$ are reported for $\delta_{u}=0.02$ and 0.2 for magnetic fields ranging from 0 to $3 \mathrm{~T}$. Here $\varepsilon_{o}$ and $t_{o}$ represent the efficiency and time per history to complete the simulation for the NULL simulation (similarly $\varepsilon_{B}$ and $t_{B}$ are for the magnetic field case). Simulation efficiency is calculated using $\varepsilon=\left(s^{2} t\right)^{-1}$, where $s$ is the simulation's estimated statistical uncertainty. The effect of $\delta_{u}$ on $\varepsilon_{n o r m}$ and $t_{\text {norm }}$ are also studied for a $1.5 \mathrm{~T}$ magnetic field. All timing and efficiency simulation are performed with $1.5 \times 10^{7}$ histories on a single cpu core.

\subsubsection{Results and discussion}

Fig. 3.12 provides the normalized efficiency and timing for the CI NE 2571 ion chamber in delrin geometry with a $1.5 \mathrm{~T}$ magnetic field for 1-PI and 3-PI methods as a function of $\delta_{u}$. Since the ratio of the uncertainties for the 1.5 T and NULL simulations remains constant for all $\delta_{u}$, the normalized timing is always a constant ratio to the corresponding efficiency for the respective integration techniques. Due to the increased computational complexity of the 3-PI method, the time for this algorithm is notably higher than for the 1-PI method. A greater difference in the normalized timing with reducing $\delta_{u}$ is seen because of the increase in the number of steps taken. A low $\delta_{u}$ value such as 0.02 incurs a penalty in the range of 2 to 3 times the NULL EGSnrc case for both the timing and the efficiency for either integration approaches. Consequently, the use of a higher $\delta_{u}$ is desirable to reduce the timing cost. A $\delta_{u}$ above 0.1 achieves the majority of the reduction in timing increase. Drawing from the ion chamber dose calculation and the Fano test results, a $\delta_{u}$ of 0.2 is selected as the recommended value for the 
3-PI calculations. Based on this $\delta_{u}$, a purely 3-PI simulation at $1.5 \mathrm{~T}$ would take $56 \%$ longer to run with a corresponding $36 \%$ drop in efficiency as compared to the NULL field simulation.

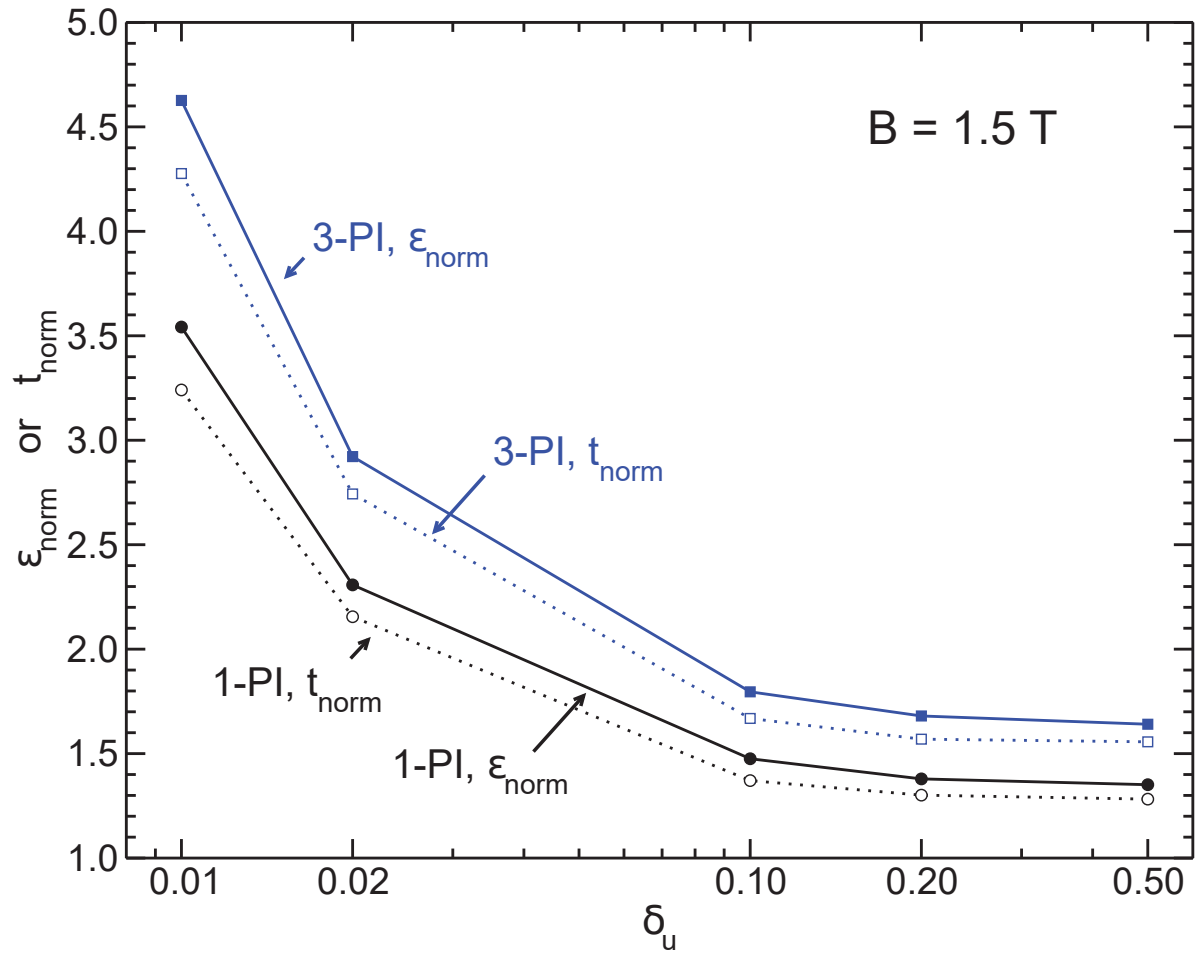

Figure 3.12: Relative timing and efficiency for an NE 2571 ion chamber in a delrin phantom in CI in the presence of a $1.5 \mathrm{~T}$ magnetic field as a function of $\delta_{u}$. Values are normalized by the same calculation without the magnetic field $\left(t_{\text {norm }}=t_{B} / t_{o}\right.$ and $\left.\varepsilon_{\text {norm }}=\varepsilon_{o} / \varepsilon_{B}\right)$.

The effect of the magnitude of the magnetic field on the normalized timing and efficiency for the ion chamber simulation is given in Fig. 3.13. The timing and the efficiency have a strong dependence on the magnetic field for a $\delta_{u}$ of 0.02 . At a $3 \mathrm{~T}$ field a 3 fold increase in the time is incurred even for the 1-PI. Moving to a $\delta_{u}$ of 0.2 reduces the dependence of the timing on the magnetic field. The statistical uncertainty in the simulation is independent of the choice of $\delta_{u}$ and is closely tied to the magnetic field used. The uncertainty for a fixed number of histories increases gradually by approximately $12 \%$ between the 0 and $3 \mathrm{~T}$ case. The variation in the uncertainty is likely related to an increased variability in track length with increasing magnitude fields. To reduce the previously quoted increase in time with the 3 -PI method at a $\delta_{u}$ of 0.2 , the "Adaptive 
Integration" and "Scaled $\delta_{u}$ " algorithms are also tested. The "Scaled $\delta_{u}$ " algorithm reduces the dependence of the timing on the magnitude of the magnetic field. The "Adaptive Integration" algorithm makes use of the best of the two integration methods, since it applies the accuracy of the 3-PI when needed and suffers only a $38 \%$ increase in simulation time at higher magnetic fields while maintaining accuracy.

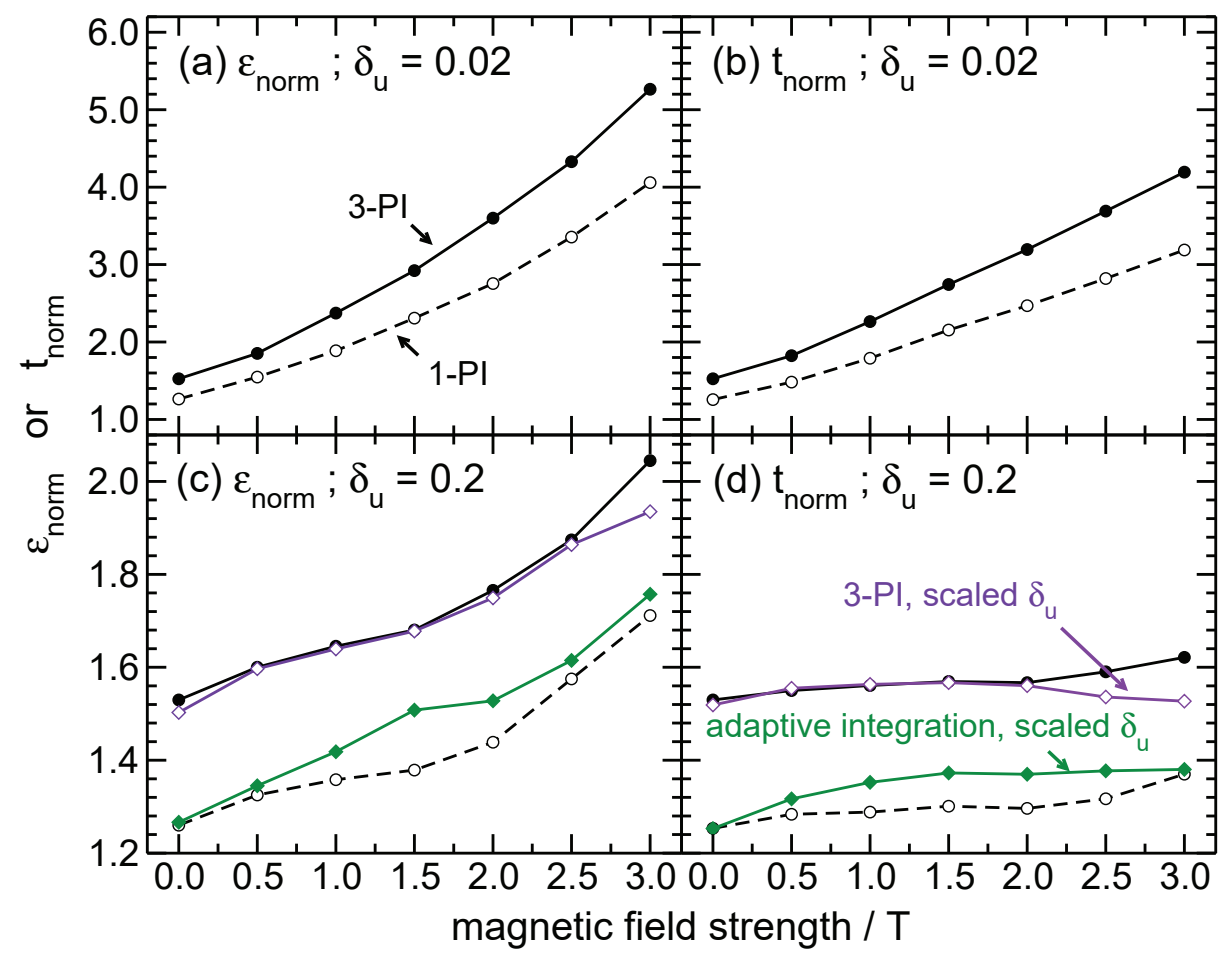

Figure 3.13: Normalized (as in Fig. 3.12) timing and efficiency for an NE 2571 ion chamber for $\delta_{u}$ of 0.02 and 0.2 as a function of magnetic field. Symbol meaning is maintained through all panels. Panels (a) and (c) are normalized efficiency and panels (b) and (d) are normalized timing. Note the difference in y-axis scales.

A timing comparison was also performed for a $10 \mathrm{~cm} \times 10 \mathrm{~cm} \times 6 \mathrm{~cm}$ phantom with $(3 \mathrm{~mm})^{3}$ voxels with an incoming parallel $4 \mathrm{~cm} \times 4 \mathrm{~cm} 6 \mathrm{MV}$ beam in DOSXYZnrc. A 1.5 $\mathrm{T}$ magnetic field is aligned perpendicular to the beam axis and directed along the negative $\mathrm{Z}$ direction. The entire phantom is filled with water. In this case the increase in the computational time is $48 \%$ compared to the NULL EGSnrc simulation with ECUT set to $861 \mathrm{keV}$, range rejection applied to electrons below $2 \mathrm{MeV}$, and a photon splitting factor of 10 . In the second test, the first $3 \mathrm{~cm}$ of the phantom are filled with water and the rest with air. There is a $76 \%$ increase in the simulation time compared 
to the NULL EGSnrc simulation for the same phantom with an ECUT of $611 \mathrm{keV}$. This difference in the increase in the computation time is attributed to the increased electron path length permitted in the air, which is affected more by the step-size restrictions implemented in the magnetic field code. Running on a single core of a 24 core Intel Xeon E5-2680 v3 2.50 GHz CPU machine, the CI simulations take approximately $6.3 \mathrm{~s}$ to achieve $2 \%$ uncertainty for the NULL case while a $1.5 \mathrm{~T}$ magnetic field simulation with the "Adaptive Integration" and "Scaled $\delta_{u}$ " algorithms turned on with a reference field of $1.5 \mathrm{~T}$ takes $9.4 \mathrm{~s}$ to obtain the same uncertainty. A simulation with a $(30 \mathrm{~cm})^{3}$ water phantom with $(3 \mathrm{~mm})^{3}$ voxels and a $4 \times 4 \mathrm{~cm}^{2} 6 \mathrm{MV}$ photon beam coming in from the negative-z direction on the same machine takes 30 minutes for the NULL case and 44 minutes for a 1.5 T magnetic field in the y-direction ("Adaptive Integration" and "Scaled $\delta_{u}$ " turned on) to achieve $2 \%$ along the central axis.

The efficiency and timing studies provided above do not disentangle the physics of the magnetic field influence from the actual complexity of the algorithm that is employed. Since the magnetic field influences the trajectory of electrons, there could be an inherent increase in simulation time even if there were no computational cost for calculating the effect of the magnetic field.

\subsection{Summary}

The magnetic field code has been validated by the Fano test as applied in a magnetic field and has been found to agree with the expected results at the $0.1 \%$ level. The increase in the computational time was found to be approximately $38 \%$ for ion chamber simulations and between $45 \%$ and $80 \%$ for a $(3 \mathrm{~mm})^{3}$ voxel phantom geometry. The increase in simulation time is found to be geometry dependent. It is well below the increase which would be incurred by the use of a $\delta_{u}$ restriction of 0.02 or less.

Agreement with experimental data ${ }^{42}$ is found at magnetic fields below $1 \mathrm{~T}$ for the 
NE 2571 ion chamber simulation. Air gaps around the chamber can induce variations in the normalized dose response on the order $1 \%$, and the tilt of the incoming beam with respect to the magnetic field can also influence the ion chamber reading. Detailed experimental descriptions and details of the chamber sensitive volume, as shown in Chapter 5, are required to adequately simulate all of the physical effects induced by the magnetic field.

This validated and tested magnetic field transport algorithm is now applied using the "full" algorithm for all of the simulations in the subsequent chapters. 


\section{Chapter 4}

\section{Magnetic field dose calculations in hetero- geneous lung models}

In this chapter the magnetic field code is applied to evaluate the importance of including lung heterogeneities in treatment planning when a magnetic field is present around the patient. Although the geometry used is fairly simple, it is clear that neglecting larger or structured heterogeneities may produce undesired dosimetric effects in the tissue. These are the results of technical report $I$.

\subsection{Introduction}

The lung is a complex and highly heterogeneous organ containing air and near water equivalent density tissue ${ }^{56}$. This often presents a challenge and means that the lung is commonly simulated as a homogeneous medium ${ }^{57-59}$ with a density ${ }^{60}$ near $0.26 \mathrm{~g} / \mathrm{cm}^{3}$. Within the context of MC methods, lung tissue is approximated using appropriate composition and density distributions. Babcock and Sidhu ${ }^{61}$ have demonstrated that, if anatomical details of the lung are incorporated into a $\mathrm{MC}$ simulation, even when there is no magnetic field, dose deviations as large as $14 \%$ from the homogeneous case occur. High resolution CT scans are capable of determining some of the lung structure but scan slice thicknesses below $1 \mathrm{~mm}$ are needed to obtain reliable models of the lung 
structure, and even then voxel averaging can reduce heterogeneity detail ${ }^{62,63}$.

The effect of magnetic fields on charged particle transport has become an important topic, particularly for dose calculations near interfaces between low and high density media (e.g., lung-tissue) $)^{8,12,16,18}$. Of particular interest is the surface dose enhancement that results from the ERE when electrons curve more in the low-density region and return to the higher-density surface. A magnetic field parallel to the incoming photon beam can reduce this effect, but this may not be clinically feasible for some MRgRT machines. CT based and simplified lung phantom studies have demonstrated that, compared to the $0 \mathrm{~T}$ case, dose differences on the order of $40 \%$ in the lung are possible when a $0.5 \mathrm{~T}$ magnetic field is present and stronger magnetic fields will produce larger deviations ${ }^{12,64,65}$. Although not evaluating the effects explicitly, a few studies had indicated that it is reasonable to expect a compound effect caused by the ERE in a heterogeneous lung structure ${ }^{18,66}$. If a homogeneous lung region or a broad ranged Hounsfield Unit density assignment is used for the MC calculations, the true dose in the magnetic field may be inaccurately determined.

Simplified lung-like phantoms with an incoming $6 \mathrm{MV}$ photon beam in $0,0.35$, 0.6, and 1.5 T magnetic fields are simulated using the EGSnrc DOSXYZnrc application along with the "Full Algorithm" magnetic field transport code described in chapter 2. The dose deposition in lungs modelled as heterogeneous or homogeneous lung substructures are compared. The heterogeneous phantom is simulated with 0.2 , 2 , or $4 \mathrm{~mm}$ water vapour gaps which are anatomically reasonable analogues to bronchus $\operatorname{diameters}^{67,68}$.

\subsection{Lung-like geometry configuration}

DOSXYZnrc is run with all default parameters except the activation of the EXACT single scatter algorithm instead of the PRESTA-I transport for boundary crossing. The 
EXACT transport option is necessary for the magnetic field macros. The skin-depth is set to 3 elastic mean free paths. ECUT is set to $521 \mathrm{keV}$ and PCUT to $10 \mathrm{keV}$.

A $20 \mathrm{~cm} \times 20 \mathrm{~cm} \times 8.8 \mathrm{~cm}$ phantom is modelled with an incoming $4 \mathrm{~cm} \times 4 \mathrm{~cm}$ $6 \mathrm{MV}$ photon beam and a magnetic field perpendicular to the photon beam is present throughout the geometry. Three materials having the same density effect correction as water are modelled. These materials are water $\left(\rho_{w}=1.0 \mathrm{~g} / \mathrm{cm}^{3}\right)$, gaseous water vapour $\left(\rho_{v}=1.2 \times 10^{-3} \mathrm{~g} / \mathrm{cm}^{3}\right)$, and lung-like water $\left(\rho_{l}=0.333 \mathrm{~g} / \mathrm{cm}^{3}\right)$. The first and the last $2 \mathrm{~cm}$ of the geometry along the z-axis are filled with water. In the homogeneous lung phantom, the $4.8 \mathrm{~cm}$ region between the water regions is filled with the lung-like water, and is divided into 48 slabs with a thickness of $1 \mathrm{~mm}$, figure $4.1 \mathrm{a}$. The heterogeneous lung phantom has two out of every three layers filled with vapour-density water and one out of three layers with regular-density water. The $4.8 \mathrm{~cm}$ region corresponding to the $\rho_{l}$ in the homogeneous phantom is filled with an alternating pattern of two $1 \mathrm{~mm}$ slabs of water vapour followed by a $1 \mathrm{~mm}$ slab of regular water, figure $4.1 \mathrm{~b}$. This configuration will be called $2 \mathrm{~mm} / 1 \mathrm{~mm}$. The lung regions in the two phantoms have the same overall density within $0.5 \%$. 


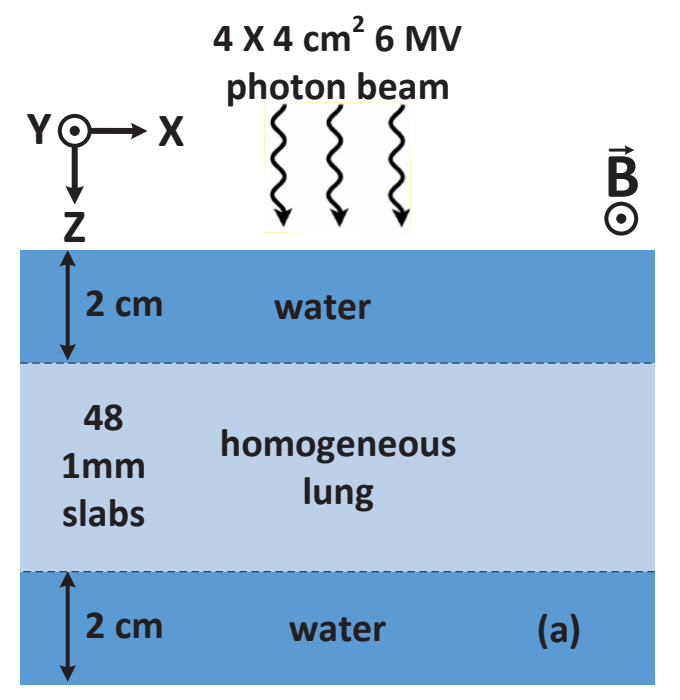

(a)

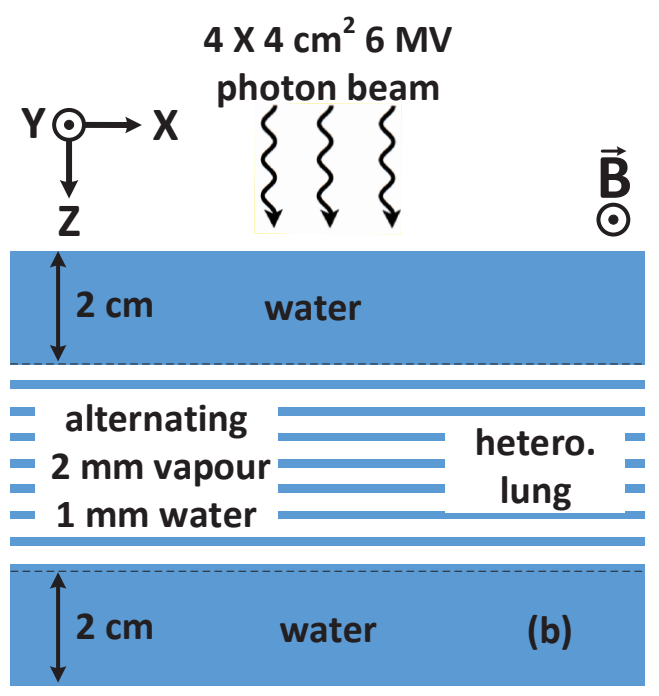

(b)

Figure 4.1: (a) Homogeneous lung phantom with lung density water. (b) Heterogeneous lung phantom with alternating water vapour and regular density water. Both are divided into 48 slabs with a thickness of $1 \mathrm{~mm}$ (not all shown and deepest regular-density slab is essentially part of the back wall).

Dose in the above phantoms is scored in $1 \mathrm{~mm}^{3}$ voxels along the $\mathrm{x}$-axis at the exit surface of the upper $2 \mathrm{~cm}$ water slab at $y=0 \mathrm{~cm}$ (i.e., in and out of the page in figure 4.1). Dose as a function of z-axis depth at $y=0 \mathrm{~cm}$ is scored at three $\mathrm{x}$ axis positions $(x=-2,0,+2 \mathrm{~cm})$. To compare the two phantoms' depth-dose curves (DDCs), ratios of the dose to the water voxels in the heterogeneous case to the dose at the same position in the homogeneous lung phantom are reported. DDCs and ratios are given from $1.7 \mathrm{~cm}$ to $7.1 \mathrm{~cm}$ in the phantom, with the first and last three dose points corresponding to the dose in $1 \mathrm{~mm}$ voxels at the interior surfaces of the $2 \mathrm{~cm}$ thick water regions.

To determine the dependence of the dose distribution on the vapour gap thickness, the heterogeneous phantom is simulated with an alternating pattern of four $1 \mathrm{~mm}$ water vapour slabs followed by two $1 \mathrm{~mm}$ water slabs ( $4 \mathrm{~mm} / 2 \mathrm{~mm}$ geometry). In this configuration, differences, caused by the electron return effect, between the entry and 
exit doses of each of the water slabs in the lung region become apparent. To explore the possibility of a similar effect in the $2 \mathrm{~mm} / 1 \mathrm{~mm}$ case, it is simulated with $0.5 \mathrm{~mm}$ thick slabs in the lung region while maintaining the overall thicknesses of the vapour and water segments. The voxel size is increased to $0.3 \mathrm{~cm} \times 2 \mathrm{~cm}$ in the $\mathrm{x}$ and $\mathrm{y}$ directions, respectively, and the z-axis dimensions are set to the thickness of the slabs in the lung region. The increased voxel size in the y-direction is justified because the magnetic field causes electrons to curve along the $\mathrm{x}$-axis, and the difference between the dose ratios of the larger and $1 \mathrm{~mm}^{3}$ voxels is below $0.5 \%$.

In addition to the slab phantom, a geometry which more closely resembles the bronchioles of the lung is simulated by filling the lung region beyond $|y| \geq 0.5 \mathrm{~mm}$ with the homogeneous lung media in the heterogeneous $2 \mathrm{~mm} / 1 \mathrm{~mm}$ geometry shown in figure 4.1b. This creates a series of rectangular $1 \mathrm{~mm}$ thick (in the x-direction) tubes of vapour and water stacked along the z-axis as shown in figure 4.2. Ratios of the water region dose in the heterogeneous phantom to homogeneous dose at the same location is calculated for $1 \mathrm{~mm}^{3}$ voxels. A single tube of $2 \mathrm{~mm}$ vapour $-1 \mathrm{~mm}$ water was also simulated at the center of the homogeneous lung phantom.

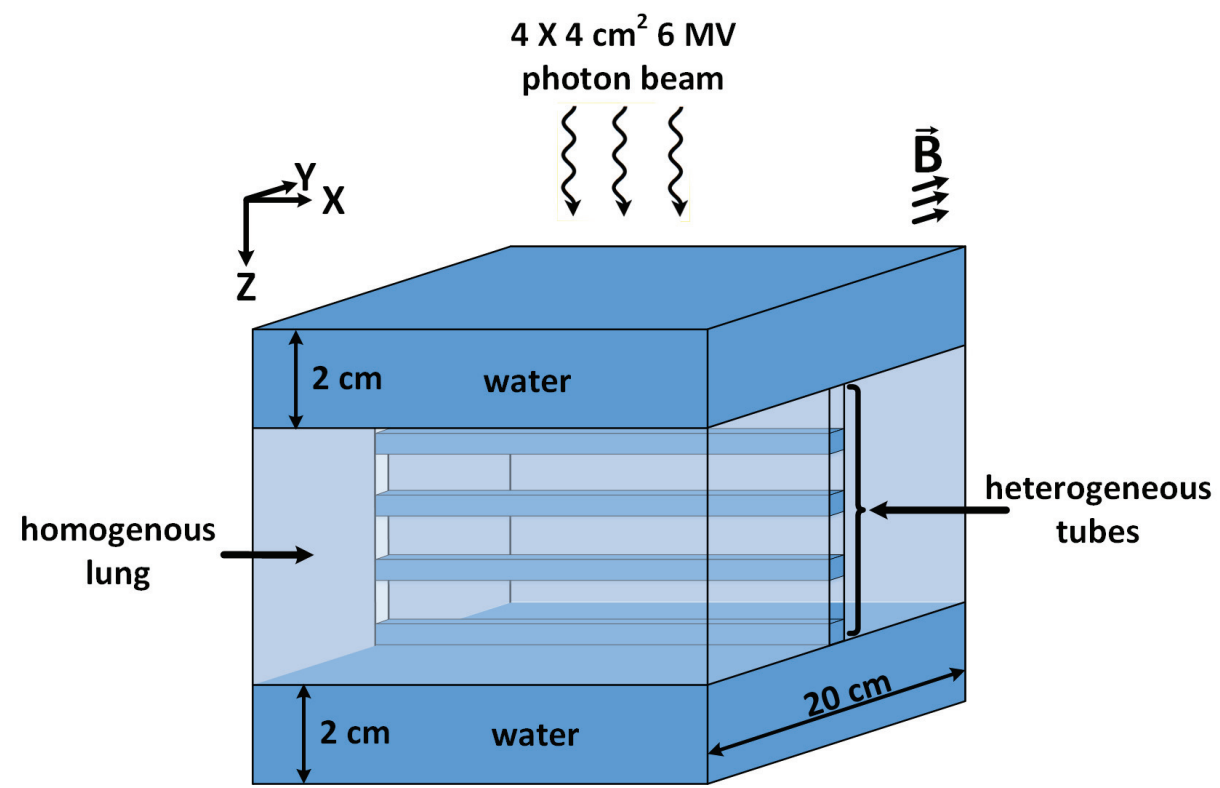

Figure 4.2: Heterogeneous tube geometry. Tube width is set to $1 \mathrm{~mm}$ and simulated with the $2 \mathrm{~mm} / 1 \mathrm{~mm}$ vapour-water configuration (not all tubes shown). 


\subsection{Results and discussion}

Figure 4.3a shows DDCs, normalized to the dose at the centre of the lung for $0 \mathrm{~T}$, for the homogeneous lung phantom as a function of field strength. The stronger magnetic fields cause a more pronounced ERE near the surfaces of the water regions while not causing notable changes within the central lung region. The DDCs in the presence of the magnetic field are consistent with other studies ${ }^{39}$.

In figure $4.3 \mathrm{~b}$, differences between having the homogeneous or the heterogeneous $2 \mathrm{~mm} / 1 \mathrm{~mm}$ case with $1 \mathrm{~mm}$ slabs in the lung region are evident in the exit surface doses of the upper $2 \mathrm{~cm}$ water slab for the $0.35,0.6$, and $1.5 \mathrm{~T}$ cases while the $0 \mathrm{~T}$ dose differences are hardly distinguishable. The known shift and enhancement of the dose distribution caused by the magnetic field are clear.

Figures $4.3 \mathrm{c}-4.3 \mathrm{e}$ are the ratios of doses in the water regions in the heterogeneous phantom to doses in the homogeneous phantom at the same location. Variations up to $17 \%$ are seen at the surfaces of the water slabs for non-zero magnetic fields. These variations are in addition to those in figure 4.3a. Near the regions of higher dose (i.e. at $x=0 \mathrm{~cm}$ and $x=2 \mathrm{~cm}$ ) the dose appears to be overestimated by the homogeneous calculation while for $x=-2 \mathrm{~cm}$ the dose is underestimated for the majority of the lung region, especially for the higher field. The results for an identical simulation with ${ }^{60} \mathrm{Co}$ are similar with fluctuations as large as $18 \%$ for the water region surface doses. The +2 and $-2 \mathrm{~cm}$ profiles show changes as large as $18 \%$ for the water region surface doses. The difference between the +2 and $-2 \mathrm{~cm}$ distributions is due to the magnetic field curving electron trajectories towards the positive x-axis, as seen the surface dose profiles in figure 4.3b. The largest differences are in regions of rapid dose variation. Changing the order of the inhomogeneity to $1 \mathrm{~mm}$ vapour - $1 \mathrm{~mm}$ water - $1 \mathrm{~mm}$ vapour (from $2 \mathrm{~mm}$ vapour - $1 \mathrm{~mm}$ water) produced slightly lower but comparable effects. 
Introducing a $(1.1 \mathrm{~cm})^{3}$ tumour $\left(\rho=1.0 \mathrm{~g} / \mathrm{cm}^{3}\right)$ to the center of the lung with $2 \mathrm{~mm}$ of vapour adjacent reveals that the front surface dose of the tumour is overestimated by the homogeneous simulation by about $10 \%$ to $13 \%$ in a 1.5 T field for the $2 \mathrm{~mm} / 1 \mathrm{~mm}$ case. Although this does not account for density variation in the tumour, it is evident that some of the largest dose differences occur in the unit density materials which are adjacent to the lung tissue.

In the dose ratio figures mentioned above, the dose to the water slabs in the lung region are averaged over the entire thickness of the slab. In figure $4.3 \mathrm{f}$ a comparison is made between a $2 \mathrm{~mm} / 1 \mathrm{~mm}$ case using $1 \mathrm{~mm}$ vs. $0.5 \mathrm{~mm}$ scoring regions. In addition to demonstrating the averaging effect, it is clear that the ERE produces sharp dose gradients causing variation of more than $10 \%$ within the individual slabs. Even larger differences are seen in the $4 \mathrm{~mm} / 2 \mathrm{~mm}$ calculations with $1 \mathrm{~mm}$ slabs. An additional calculation for a $0.2 \mathrm{~mm} / 0.1 \mathrm{~mm}$ geometry with $0.1 \mathrm{~mm}$ slabs did not produce noticeable changes between the homogeneous and heterogeneous phantoms even near the surfaces of the water regions. This is to be expected since reducing the thickness of the gas vapour region should result in a calculation similar to the homogeneous geometry. 


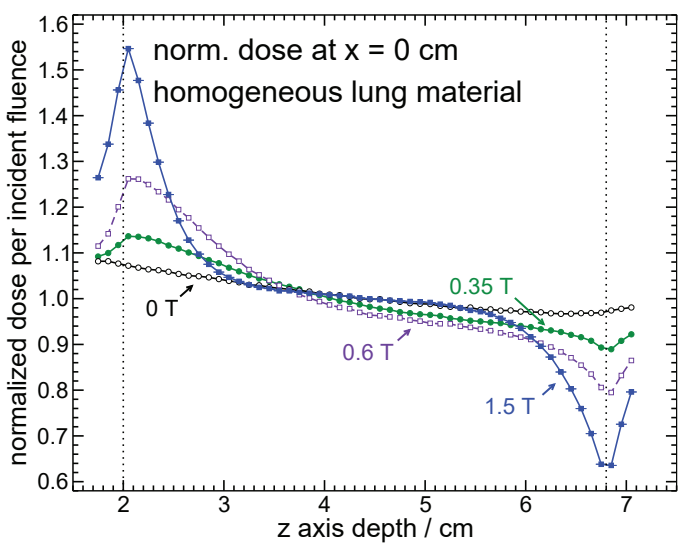

(a) DDCs at $x=0 \mathrm{~cm}$ normalized by the dose at the center of the lung for $0 \mathrm{~T}$. Normalized by $6.3327 \times 10^{-12}$ Gy $\mathrm{cm}^{2}$.

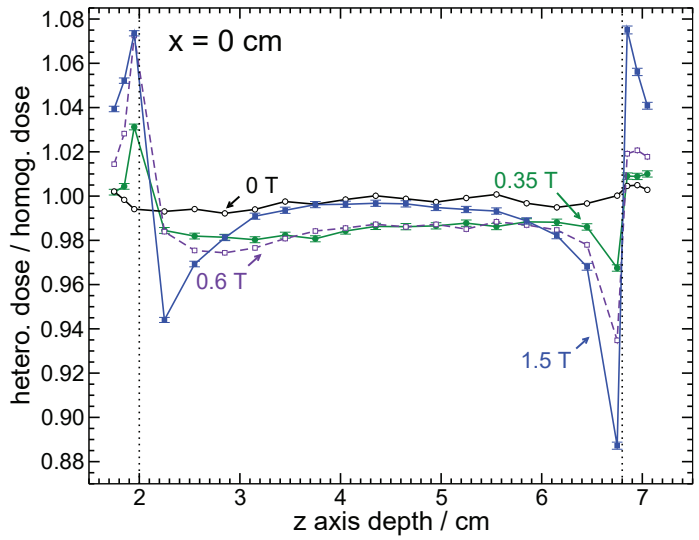

(c) Hetero. to homog. dose ratios along the central axis for the same incident fluence.

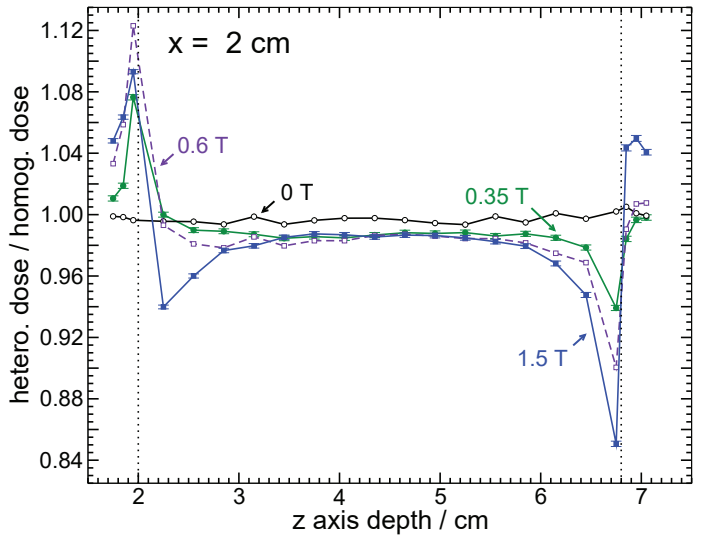

(e) As (c) but at $x=2 \mathrm{~cm}$.

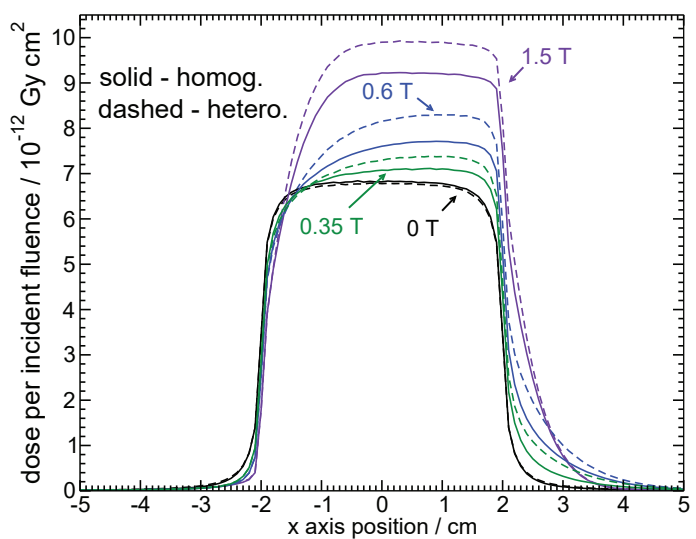

(b) Dose per incident fluence to the exit surface of the upper water slab along the $x$-axis at $y=0$ and $z=2 \mathrm{~cm}$.

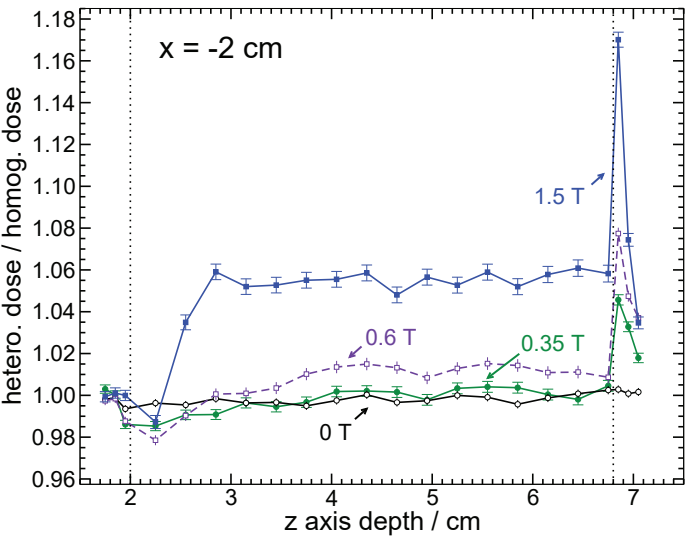

(d) As (c) but for $x=-2 \mathrm{~cm}$.

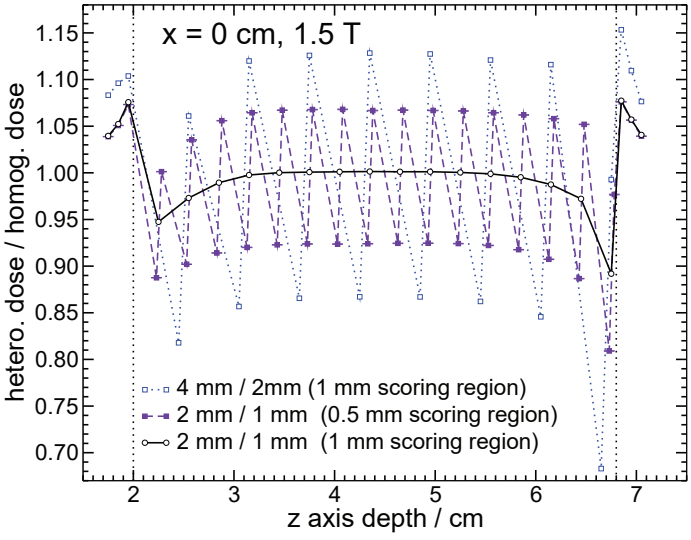

(f) As (c) but for different vapour gaps and voxel size.

Figure 4.3: Dose per incident fluence and ratios for lung simulations. Thin dotted vertical lines represent the edges of the $2 \mathrm{~cm}$ water slabs. (a) to (e) are $2 \mathrm{~mm} / 1 \mathrm{~mm}$ with $1 \mathrm{~mm}^{3}$ voxels. 
Figure 4.4 provides the central axis DDC ratios for the tube-like geometry for the various magnetic field magnitudes. Slight reductions from figure $4.3 \mathrm{c}$ can be seen in the magnitudes of the surface deviations, but the overall characteristics of the effect are maintained. The observed differences are expected as this model is much closer to the fully homogeneous simulations, and it is possible that the true structure of the lung would wash out some of the observed variations in the slab geometry. Further, dose variations ranging from $3.6 \%$ to $7.2 \%$ are seen near the surfaces of the single tube simulation. These results indicate that individual inhomogeneous structures have the potential of inducing large dose difference in a magnetic field.

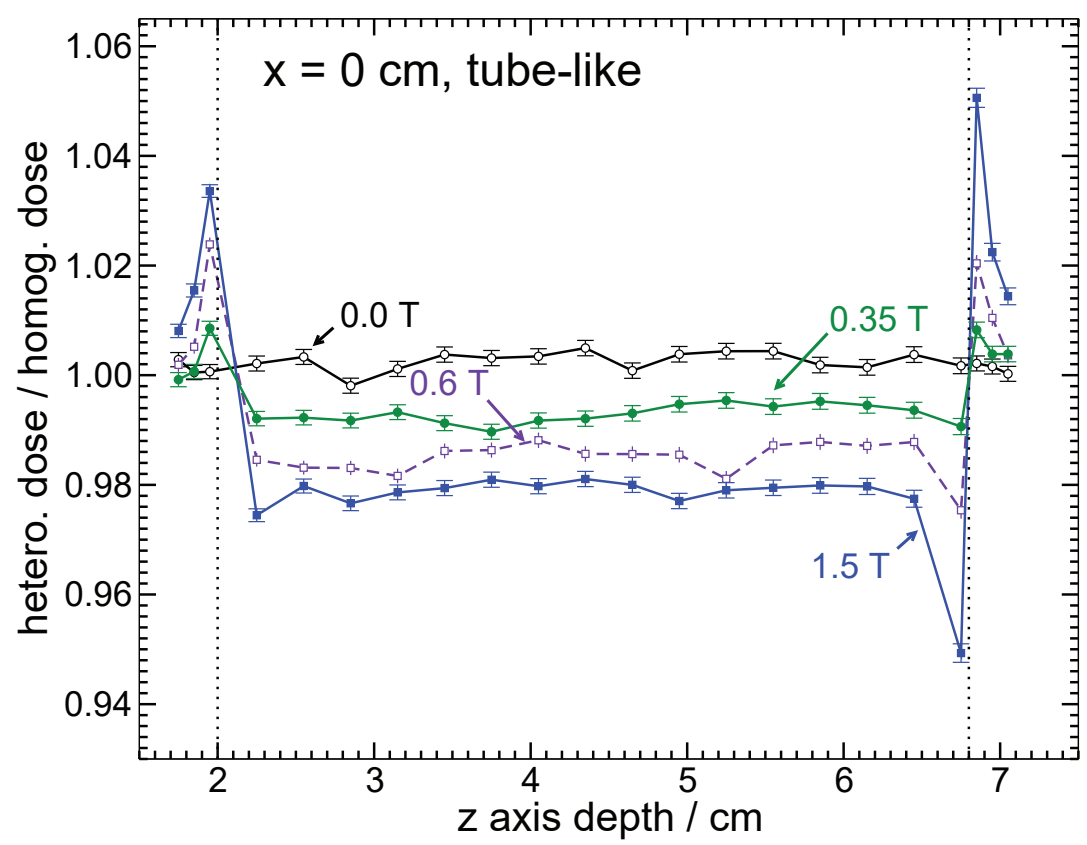

Figure 4.4: Heterogeneous to homogeneous DDC ratio for the tube-like geometry.

\subsection{Summary}

There are substantial inaccuracies in the doses to the lung tissue itself, due to using a homogeneous lung model in a magnetic field which is how many systems treat a CT scan with limited resolution. Variations between approximately $3 \%$ and $18 \%$ are seen near the tissue/lung interfaces when a simple model of the inhomogeneous nature of the 
lung is accounted for in the presence of a $0.35,0.6$ or $1.5 \mathrm{~T}$ magnetic fields. Within the lung-like media, differences on the order of several percent and reaching $6 \%$ in some cases are observed. The dose to the beam facing surface of a water density equivalent tumour within the lung geometry was found to be overestimated by a homogeneous phantom calculation by nearly $13 \%$. This demonstrates that the heterogeneous nature of the lung has a significant effect on the dose delivered to homogeneous tissues nearby. Simulations which more closely approximate the bronchiole geometry demonstrate that the true structure of the lung can smear out some of the observed variations, and hence detailed modelling of the lung will be needed to better ascertain the effect. In the absence of a magnetic field, the inhomogeneity did not produce any distinguishable dose differences in our simple models although previous work suggest an effect even then ${ }^{61}$. Since dose tolerances are based on $0 \mathrm{~T}$ fields where the homogeneous approximation is valid, care must be taken when magnetic fields are present since some regions will receive significantly higher doses.

Although the model used is far from an exact representation of the lung, these calculations demonstrate that lung structure may need to be included in dose calculations in the presence of a magnetic field. We observe that bronchus diameters below $1 \mathrm{~mm}$ may not cause large dose variations but larger air regions can cause dose fluctuation which a homogeneous model would miss. Reduction of the effect of the heterogeneities is likely in regions with beams incident from different directions, but this will not be the case near the entrance to the lung in most cases. Care must be taken in selecting $\mathrm{CT}$ resolution and the size of the scoring region for magnetic field $\mathrm{MC}$ calculations due to the possibility of volume averaging effects. Overall, additional and careful analysis of treatment planning and calculation of lung doses for radiation delivered in the presence of magnetic fields is necessary. 


\section{Chapter 5}

\section{Sensitive volume effects on ion chamber re- sponse in magnetic fields}

This chapter addresses the effect of the selection of the sensitive air volume used in the Monte Carlo cylindrical ion chamber model on the calculated dose when a magnetic field is present. This problem impacts the majority of published comparisons of Monte Carlo ion chamber simulations in magnetic fields. These are the results of the peer-reviewed paper II.

\subsection{Introduction}

Experimental measurements of ion chamber response as a function of magnetic field normalized by the no magnetic field case have been reported by several groups ${ }^{20,31,42}$. These measurements are compared to MC simulations with the goal of justifying the use of the MC models for future calculations of correction factors ${ }^{26,29}$, as was done in chapter 3. As in ion chamber simulations in the absence of magnetic fields ${ }^{34,69}$ the ion chamber cavity's geometric volume is often used as the sensitive volume in place of the unknown smaller true collection volume. Numerical calculations have shown distortions in the charge collecting electric field of the chamber near the stem, where the geometric sensitive volume usually begins ${ }^{70,71}$. Since it is the electric field that determines charge 
collection, such distortions in the field correspond to insensitive regions in which charge is not gathered by the central electrode. The excluded volume for several chambers, when accounting for the electric field, represents between about $8 \%$ and $20 \%$ of the geometric sensitive volume ${ }^{70,71}$. Additional variations in the volume are seen based on any differences in potential between the guard and central electrode ${ }^{70}$. Further, experimental evaluation of dosimetric response maps of several ion chambers indicate a drop in sensitivity in the same region near the stem ${ }^{72}$. This effect does not usually appear in MC calculations since these omit the complex structure of the electric field inside the ion chamber. Although the reduced volume would cause a reduction in the total collected charge, any experimentally or computational determined dose ratio in the absence of a magnetic field should not be affected by the volume reduction, as demonstrated by the good agreement between calculated and measured $k_{Q}$ factors for many ion chambers ${ }^{73}$.

Considering that the magnetic field sweeps electrons in a preferential direction, there can be a notable change in the calculated chamber dose. In this study, the sensitive volume of five ion chambers is varied and the corresponding response is evaluated as a function of magnetic field.

\subsection{Set-up of sensitive volume simulations}

The egs_chamber ${ }^{52}$ application is used to simulate the PTW $30013\left(0.607 \mathrm{~cm}^{3}\right)$, PTW 31006 $\left(0.0146 \mathrm{~cm}^{3}\right)$, PTW $31010\left(0.275 \mathrm{~cm}^{3}\right)$, Exradin A12S $\left(0.246 \mathrm{~cm}^{3}\right)$, and Exradin A1SL $\left(0.057 \mathrm{~cm}^{3}\right)$ chambers inside a $26 \mathrm{~cm}$ long, $2 \mathrm{~cm}$ wide, and $5 \mathrm{~cm}$ high PMMA phantom. The volumes given in brackets are the nominal geometric sensitive volumes. As in the NPL phantom of Agnew et al. ${ }^{31}$, a $1.5 \mathrm{~cm}$ diameter and $14.5 \mathrm{~cm}$ long water cavity in which the chambers are positioned is also simulated. As in Figure 5.1, the chambers are oriented to point in the $\mathrm{x}$-direction, while a Co- $60^{74}$ or $7 \mathrm{MV}^{65}$ photon beam, located at 
an SSD of $162 \mathrm{~cm}$, is incident from the negative z-direction. A 0 to $2 \mathrm{~T}$ magnetic field is simulated in the negative y-direction. This orientation, in which the ion chamber, photon field, and magnetic field are all perpendicular to each other has become the most common geometric configuration used in experiments ${ }^{20,31,42}$. Major advantages of the recently published Agnew et al. data, which uses this same orientation, are the use of the known Cobalt-60 source, elimination of air gaps around the chamber, and a full description of the phantom. For this reason this specific configuration is simulated and results are compared to these data. Additionally, true variance reduction techniques $^{52}$ are used to improve MC simulation efficiency and all default parameters of the egs_chamber simulation are used (NIST bremsstrahlung cross-sections are used). PCUT and ECUT are set to $10 \mathrm{keV}$ and $521 \mathrm{keV}$, respectively.

To evaluate the sensitivity of the chamber response in the magnetic field on the collection volume, the dose per unit incident photon fluence to the geometric sensitive volume with $0,0.5$, or $1 \mathrm{~mm}$ of the volume away from the stem excluded is calculated for each of the chambers. In Figure 5.1, region (1) corresponds to the first $0.5 \mathrm{~mm}$ away from the stem, and region (2) is the second $0.5 \mathrm{~mm}$. In this way, the combined volume of regions (1), (2), and (3) correspond to the total geometric sensitive volume that is normally used in MC calculations. Region (3) is the volume with the volume corresponding to $1 \mathrm{~mm}$ of chamber length away from the stem removed, and regions (3) and (2) combined are the volume with $0.5 \mathrm{~mm}$ removed. The volume fraction of the excluded air is comparable to that mentioned above (8-20\%) and found through detailed calculations of the actual collecting volume based on the electric field in the chamber $^{70,71}$. The chamber response, i.e., the average dose for the given sensitive volume per unit incident photon fluence, is evaluated as a function of magnetic field and normalized by the $0 \mathrm{~T}$ simulation for each chamber and collection volume. The ratios of the chamber responses and the associated statistical uncertainties $(k=1)$ are reported as a percent value away from unity. The overall uncertainty on the MC simulations is 
approximately $0.5 \%(\mathrm{k}=1)$, based on a $0.1 \%$ uncertainty on the transport mechanics as shown by previously shown Fano test results, Chapter 3, and a conservative uncertainty estimate of $0.38 \%$ on chamber dose ratios due to uncertainty in the photon and electron cross-sections ${ }^{69}$, and $0.25 \%$ uncertainty in the constancy of $(\mathrm{W} / \mathrm{e})_{\text {air }}$, the average energy lost per Coulomb of charge released by electrons in air ${ }^{75}$.
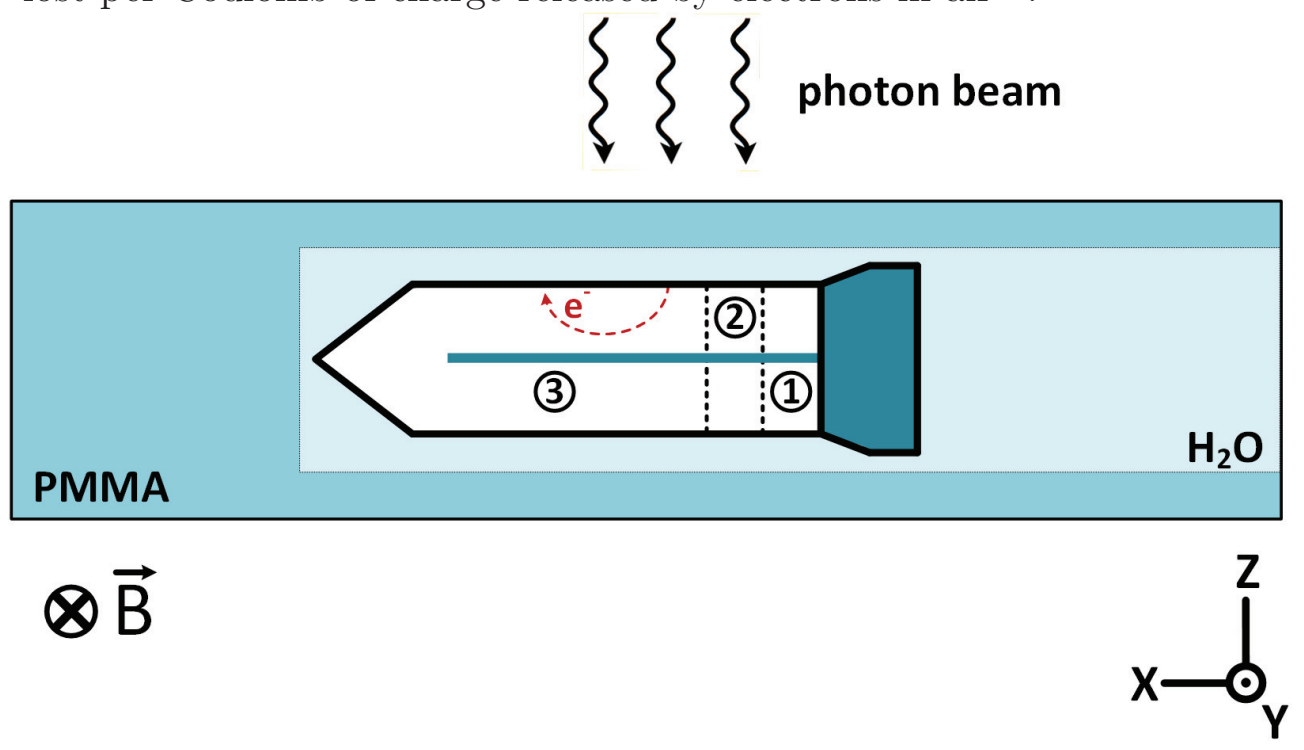

Figure 5.1: Generic ion chamber simulation geometry (dimension not to scale). Regions (1) and the combined (1) and (2) are the excluded volumes corresponding the $0.5 \mathrm{~mm}$ and $1 \mathrm{~mm}$ regions away from the stem, respectively. The curved dashed line represents the curvature of a typical incoming electron.

As seen in Figure 5.1, in the simulated orientation the magnetic field produced a clockwise rotation of negatively charged particles. This sweeps electron away from stem region and produces a regional dose enhancement near the tip of the chamber, on the left in the figure. The dose gradient caused by the presence of the magnetic field in this orientation can be seen in Fig. 6.7 in Chapter 6.

\subsection{Results and discussion}

The normalized chamber doses are given in Figure 5.2, and demonstrate that there is a notable variation in chamber response based on the sensitive volume of the chamber. 
The chamber volumes, fractions of the volume corresponding to the excluded regions, as well as the maximum deviation from the $0 \mathrm{~mm}$ simulations are provided in Table 5.1. Larger volume chambers, such as the PTW 30013 and Exradin A12S, experience a lower variation from the $0 \mathrm{~mm}$ simulation compared to the smaller volume chambers. This is to be expected since the excluded distance away from the stem was maintained constant which means that volume fractions of the excluded regions are smaller for the larger chambers.

Table 5.1: Geometric sensitive volumes (V), cavity radii (R) and lengths (L) of the ion chambers and the corresponding percent of the volumes excluded. The maximum variation from the $0 \mathrm{~mm}$ simulations and the magnetic field (B) at the maximum are also given.

\begin{tabular}{|c|c|c|c|c|c|c|}
\hline \multirow[t]{2}{*}{ Chamber $\left[\mathrm{V}\left(\mathrm{cm}^{3}\right), \mathrm{R}, \mathrm{L}(\mathrm{mm})\right]$} & \multicolumn{2}{|c|}{ Volume excluded (\%) } & \multicolumn{3}{|c|}{ Max variation(\%) } & \multirow[t]{2}{*}{ B B at max. (T) } \\
\hline & $0.5 \mathrm{~mm}$ & $1 \mathrm{~mm}$ & $0.5 \mathrm{~mm}(\mathrm{Co}-60)$ & $1 \mathrm{~mm}(\mathrm{Co}-60)$ & $1 \mathrm{~mm}(7 \mathrm{MV})$ & \\
\hline PTW $30013(0.607,2.75,23.38)$ & 0.8 & 1.8 & $0.28 \pm 0.04$ & $0.61 \pm 0.04$ & $0.46 \pm 0.05$ & 0.75 \\
\hline PTW $31010(0.125,2.75,6.5)$ & 8.6 & 17.2 & $1.75 \pm 0.03$ & $3.38 \pm 0.03$ & $1.93 \pm 0.06$ & 0.75 \\
\hline PTW $31006(0.0146,1,5.09)$ & 10.7 & 21.4 & $1.64 \pm 0.09$ & $2.88 \pm 0.10$ & $1.42 \pm 0.10$ & $1.25(1$ at $7 \mathrm{MV})$ \\
\hline Exradin A12S $(0.246,3.04,10.02)$ & 4.6 & 8.9 & $0.26 \pm 0.08$ & $0.52 \pm 0.08$ & $0.49 \pm 0.07$ & 0.5 \\
\hline Exradin A1SL $(0.057,2 ., 5.93)$ & 9.4 & 18.8 & $1.74 \pm 0.05$ & $3.39 \pm 0.06$ & $1.97 \pm 0.08$ & 1 \\
\hline
\end{tabular}

The maximum variation in response from the $0 \mathrm{~mm}$ simulation appears to change in line with a change in volume (roughly doubling when comparing the $1 \mathrm{~mm}$ results to the $0.5 \mathrm{~mm}$ maximum variation). The three smallest chambers, PTW 31010, PTW 31006, and Exradin A1SL, show the largest variation in response with a maximum of $3.3 \pm 0.06 \%$ with the volume of $1 \mathrm{~mm}$ away from the stem removed. Interestingly, the PTW 31006, although the smallest chamber and the one with the largest volume fraction associated with the excluded regions, does not produce the largest variation in response of these three chambers. However, the PTW 31006 has the smallest sensitive volume radius of the three chambers and therefore produces more confinement of the curving electrons. This would reduce the dose variation from the stem to tip of the chamber, and lessen the impact of excluding a portion of the volume. Further, chambers with similar radii achieve their maximum variation at similar magnetic field values, and larger radii correspond to smaller magnetic fields at maximum variation (since weaker magnetic 
fields are associated with larger gyroradii). Past the maximum, variations from the $0 \mathrm{~mm}$ simulation begin to decrease, likely linked to the decreasing gyroradius allowing for nearly complete rotations within the volume of the chamber. It is important to note that reduction in the sensitive volume results in an increase in the relative chamber response in most cases. This indicates that the region near the tip of the ion chamber has a higher average dose than the excluded segment when a magnetic field is present. This is a result of the clockwise rotation of negatively charged particle trajectories induced by the magnetic field. This causes some electrons to leave the insensitive region and enter the sensitive region. Based on this, a magnetic field oriented in the positive ydirection would produce a counter-clockwise rotation of the electron trajectories which would lead to a higher average dose on the right side of the chamber near the stem. An orientation between these two extremes may negate the effect of the varying collection volume on the MC dose calculation, and this merits detailed further investigation.

Three of the simulated chambers demonstrate large variations, from the $0 \mathrm{~mm}$ calculations, in response as a function of magnetic field. For the PTW 31006 and PTW 31010, the $0.5 \mathrm{~mm}$ calculations appear to be much more closely aligned with the experiment than the $0 \mathrm{~mm}$ calculation. The A1SL and A12S appear to have a tighter agreement with experiment even with little reduction in sensitive volume, and the PTW 30013 simulations may indicate that a larger change in the sensitive volume is required or another effect is at play to account for the deviation from experiment for this chamber. Ultimately, the $0.5 \mathrm{~mm}$ and $1 \mathrm{~mm}$ volume boundaries serve as a very rough surrogate to true insensitive region of the chamber and a proper comparison with experimental results requires a detailed calculation of the electric field in each chamber model. Most importantly, these results demonstrate that the MC calculated ion chamber doses in magnetic fields are often sensitive to the true sensitive volume of the chamber, and the variation in relative response from the geometric sensitive volume dose calculation is strongly chamber dependent. 
Agnew et al. ${ }^{31}$ had used different PMMA phantoms for some of the chambers in their experiments. To ensure that results between phantoms are comparable, the group showed that inter-phantom variation of the relative response was near $0.1 \%$ for the PTW 30013. In the current work a comparative simulation with the PTW 31010 using the alternative phantom geometry from the experimental work shows that the relative response changes by less than $0.3 \%$ from the results in Figure 5.2. The goal is to evaluate the influence of the sensitive volume on the relative response, and for simplicity all chambers are simulated in the same phantom.

Substituting the Co-60 source with a 7 MV MR-linac spectrum source in the PTW 30013, PTW 31010, and A1SL simulations, Figure 5.2f, reveals that the sensitive volume remains an influencing component of the calculated chamber relative response. The maximum variation from the $0 \mathrm{~mm}$ simulation is slightly reduced compared to the Co60 simulations (Table 5.1) and is likely due to the increased energies of the electrons. These electrons would have a larger radius of curvature in the magnetic field, and the dose distribution in the chamber would become more uniform.

\subsection{Summary}

These results demonstrate that substantial variations in ion chamber relative response as a function of magnetic field can occur depending on the size of the chamber's collection volume. These variations are more pronounced in smaller volume ion chambers, and are found to be, for a Co-60 source, as large as $1.75 \pm 0.03 \%$ and $3.39 \pm 0.06 \%$ when excluding the volume corresponding to either $0.5 \mathrm{~mm}$ or $1 \mathrm{~mm}$, respectively, of the length away from the stem. The effect is slightly reduced, but still non-negligible, when a higher-energy $7 \mathrm{MV}$ photon spectrum is simulated.

Although the simulated variations in the chamber's sensitive volume do not exactly reflect the true collection volume that is governed by the electric field established in 
the chamber, this study demonstrates the large sensitivity of MC dose calculations to these details. Differences between the MC and the experimental results are seen for the $0 \mathrm{~mm}$ calculation, and, while a reduction in the sensitive volume does improve the agreement for some chambers, there are still some discrepancies seen even for the $1 \mathrm{~mm}$ simulations. This indicates that to properly compare many of the experimental studies of ion chambers in magnetic fields to MC calculations, a better knowledge of the true sensitive volume is required. The true collection volume could be determined by performing a detailed calculation of the electric field ${ }^{70,71}$. This would have to be done for each chamber and would be complicated by changes in volume resulting from changes in applied potential ${ }^{70}$. A more general solution for clinical dosimetry would be to find orientations in which the effect of the sensitive volume on the chamber relative response is minimized, as is shown in the subsequent chapters. To the extent that volume averaging of detector signal plays a role in small-field dosimetry, knowledge of the details of the sensitive volume may also be important in the absence of a magnetic field. 

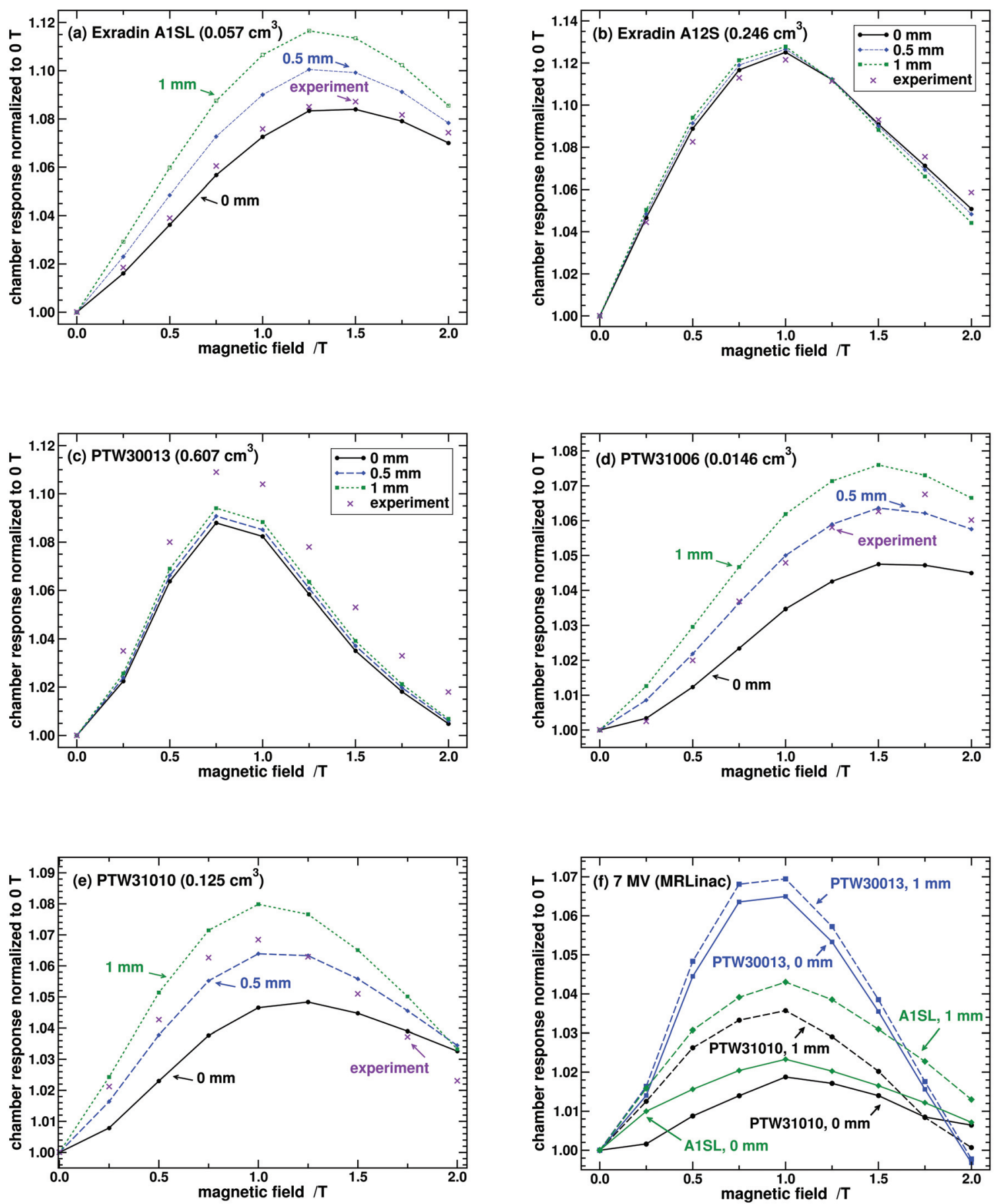

Figure 5.2: Chamber response, normalized to $0 \mathrm{~T}$, as a function of magnetic field strength with either the full sensitive volume or with the volume corresponding to excluding either $0.5 \mathrm{~mm}$ or $1 \mathrm{~mm}$ of length away from the stem. Results are for a Co-60 beam except for (f) which uses a 7 MV spectrum source for the Elekta MR-linac. Experimental results are from Agnew et al. ${ }^{31}$. Simulation statistical uncertainties $(\mathrm{k}=1)$ are smaller than the symbols, and the experimental uncertainty is $0.5 \%{ }^{31}$. 


\section{Chapter 6}

\section{Optimal orientation for clinical reference dosime- try in magnetic fields}

This chapter deals with the variation in the magnetic field and beam quality correction factor, $k_{Q}^{m a g}$, as a function of angle. The goal of this study is to determine an optimal orientation to use for clinical reference dosimetry in magnetic fields. As shown in the results below, certain orientations reduce the influence of the magnetic field and eliminate the issues of the unknown collection volume. These are the results of the peer-reviewed paper III.

\section{1 $\mathbf{k}_{Q}^{m a g}$ as a function of angle}

As shown in chapter 5 , the true sensitive volume of the cylindrical ion chamber becomes an important component of the MC simulation when a magnetic field is present. If the incorrect sensitive volume is used, the impact on the calculated magnetic field correction factors, $\mathrm{k}_{Q}^{m a g}$ or $\mathrm{k}_{B}$, will be proportional to the differences seen in chapter 5 . Here, the variation of $\mathrm{k}_{Q}^{\mathrm{mag}}$ is determined as a function of angle, and the subsequent section deals with the effect of the sensitive volume on $\mathrm{k}_{Q}^{\text {mag }}$ at these same angles.

Figure 6.1 provides the geometric set-up for calculating $\mathrm{k}_{Q}, \mathrm{k}_{Q}^{\text {mag }}$, and $\mathrm{k}_{B}$. Although only $\mathrm{k}_{Q}^{m a g}$ will be evaluated in this chapter, the variations seen in this quantity as a 
function of angle will directly translate into variation in $\mathrm{k}_{B}$. Each of the quantities in Eq. 1.8 is evaluated at a depth of $10 \mathrm{~cm}$ in a $30 \times 30 \times 30 \mathrm{~cm}^{3}$ water phantom. The water dose is determined in a $1 \mathrm{~cm}$ radius and $0.025 \mathrm{~cm}$ thick water disk. Simulations with a $0.1 \mathrm{~cm}$ radius water disk produced no statistically significant variations in the determined correction factors. Photon spectra sources, incoming from the z-direction, are used in all simulations and a field size of $10 \times 10 \mathrm{~cm}^{2}$ at the surface of the water phantom is simulated. The SSD for the ${ }^{60} \mathrm{Co}$ simulations is set to $100 \mathrm{~cm}$, and it is $133.5 \mathrm{~cm}$ for all other energies. The $133.5 \mathrm{~cm}$ SSD is chosen based on capabilities of existing MRgRT machines. Changes in SSD do not produce significant differences in the correction factors since correction factors consist of a ratio of water to chamber doses which are determined at the same SSD.
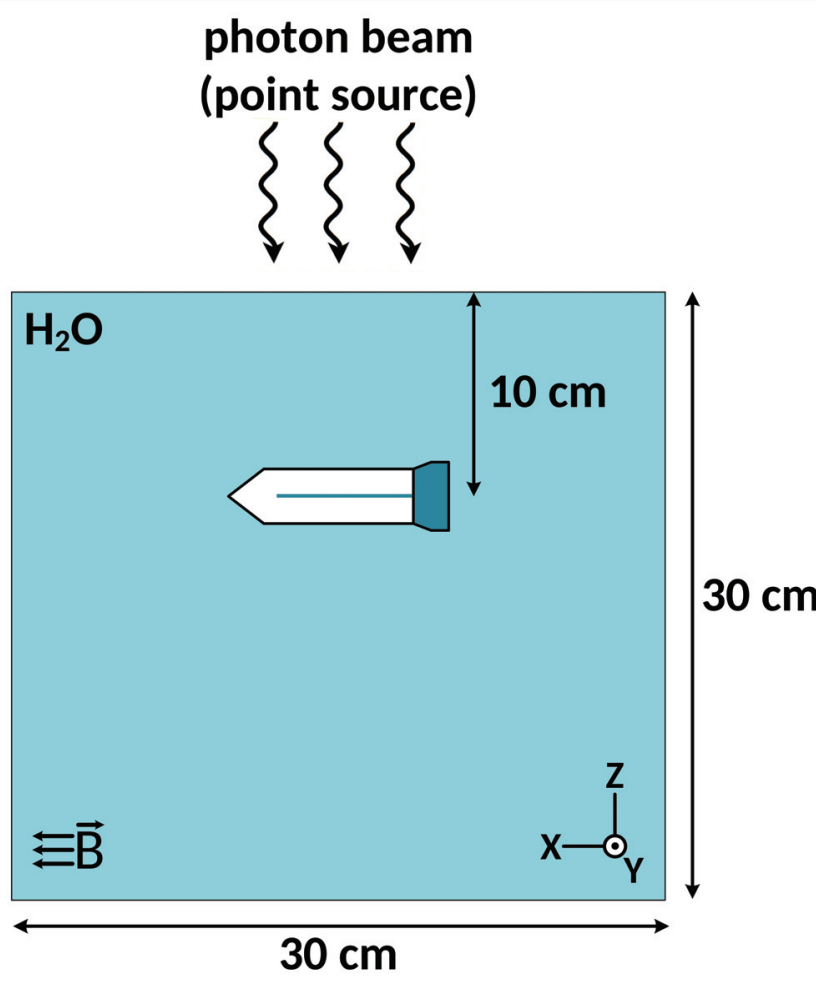

Figure 6.1: Monte Carlo geometrical set-up for correction factor calculations. The ion chamber, initially oriented in the x-direction, is positioned at a depth of $10 \mathrm{~cm}$ in a $30 \times 30 \times 30 \mathrm{~cm}^{3}$ water phantom. The magnetic field, when non-zero, is oriented in the $\mathrm{x}$-direction. The photon source is a point source with an SSD of $100 \mathrm{~cm}$ for the reference ${ }^{60} \mathrm{Co}$ and $133.5 \mathrm{~cm}$ for all other beam energies. The field size at the phantom surface is $10 \times 10 \mathrm{~cm}^{2}$. 
The ion chamber is positioned initially with the tip pointing along the x-axis. The magnetic field, when it is non-zero, is always directed in the $\mathrm{x}$ direction. To evaluate the influence of the ion chamber orientation with respect to the magnetic field on $\mathrm{k}_{Q}^{\operatorname{mag}}$, the ion chamber is rotated in the $\mathrm{x}-\mathrm{y}$ plane. Figure 6.2 looks at the chamber from the photon beam's eye view. The chamber is rotated counter-clock wise in $10^{\circ}$ increments, and $\mathrm{k}_{Q}^{m a g}$ is determined at each of these orientations. Since the water disk is symmetric, the dose-to-water is evaluated for only one orientation with the magnetic field directed along the x-axis. For $0 \mathrm{~T}$ in a ${ }^{60} \mathrm{Co}$ beam, the dose-to-air in the chamber is determined only for the $0^{\circ}$ orientation to save on computation time. Test calculations demonstrated that, as expected, there is no variability in the dose-to-air in the chamber as a function of angle in the absence of the magnetic field. Figure 6.2 highlights the four cardinal angles since these are the natural positions to perform clinical reference dosimetry in magnetic fields. The chamber would be oriented either parallel (0 or $\left.180^{\circ}\right)$ or perpendicular (90 or $270^{\circ}$ ) to the magnetic field.

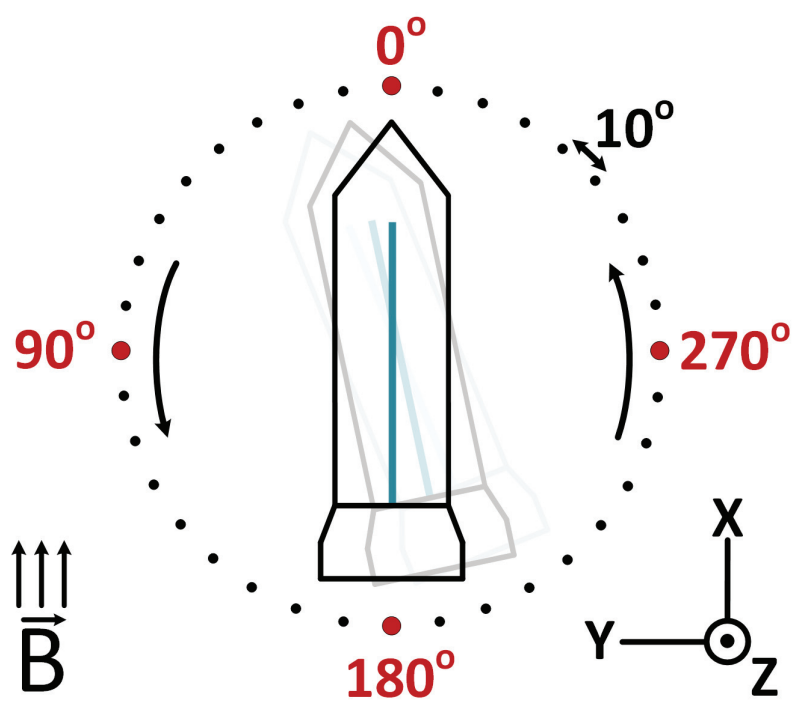

Figure 6.2: Beam's eye view of the chamber positioned in the water phantom presented in Figure 6.1. The chamber is rotated counter-clockwise in $10^{\circ}$ increments. The 0 , 90,180 , and $270^{\circ}$ orientations are highlighted as these correspond to the most likely orientations for performing reference dosimetry in magnetic fields. 
In Figure 6.3 each of the cardinal angles from Figure 6.2 is assigned to a configuration labelled I-IV. Configuration V is for a system set-up in which the magnetic field is parallel to the incoming photon beam. This notation is not related to that used in chapter 3. In each of the panels in Figure 6.3, the direction of the magnetic force on an electron travelling down the page (the direction of the incoming photon beam) is given. The direction of the magnetic field is also shown. It can be seen that for configurations I and III (C-I and C-III), the effect of the magnetic field is to curve the electron along circular cross-section of the ion chamber and not along the length of the chamber. Due to the symmetry of the magnetic field's influence at these two orientations, similar $\mathrm{k}_{Q}^{\text {mag }}$ values are anticipated for C-I and C-III. For C-II and C-IV, the effect of the magnetic field is to preferentially sweep the electrons either towards the tip or the stem of the chamber. Different $\mathrm{k}_{Q}^{\text {mag }}$ values can be expected between C-II and C-IV because of the geometric differences at each end of the cavity and due to a possible insensitive region near the stem. In $\mathrm{C}-\mathrm{V}$, the magnetic force on an electron travelling down the page is zero, and therefore the overall effect of the magnetic field is minimal for this configuration, but it is not necessarily completely absent since not all secondary electrons will travel in straight lines and in the same direction. C-V is omitted for this angular study, and is included in this section for completeness. 

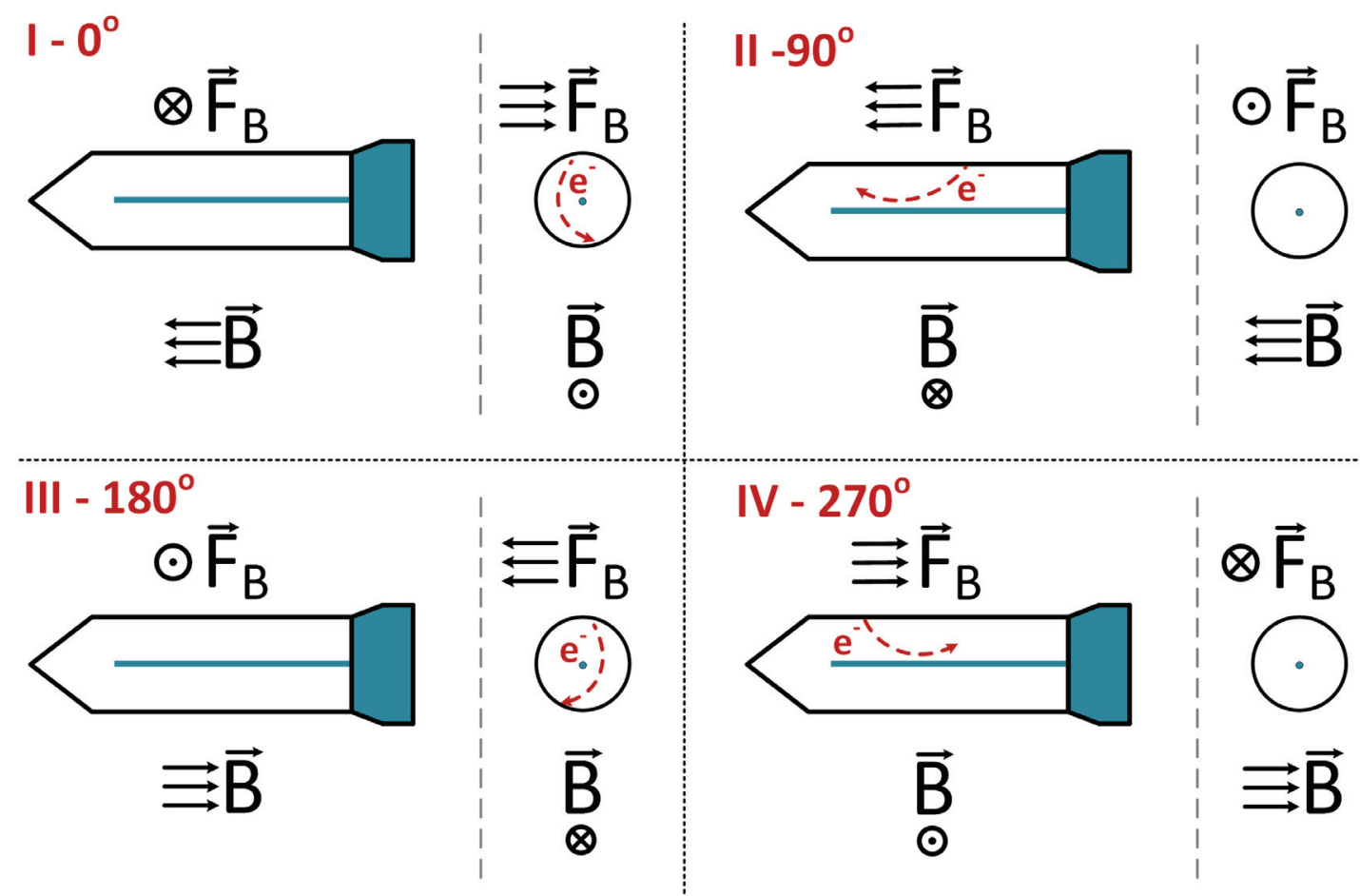

IV $-270^{\circ}$
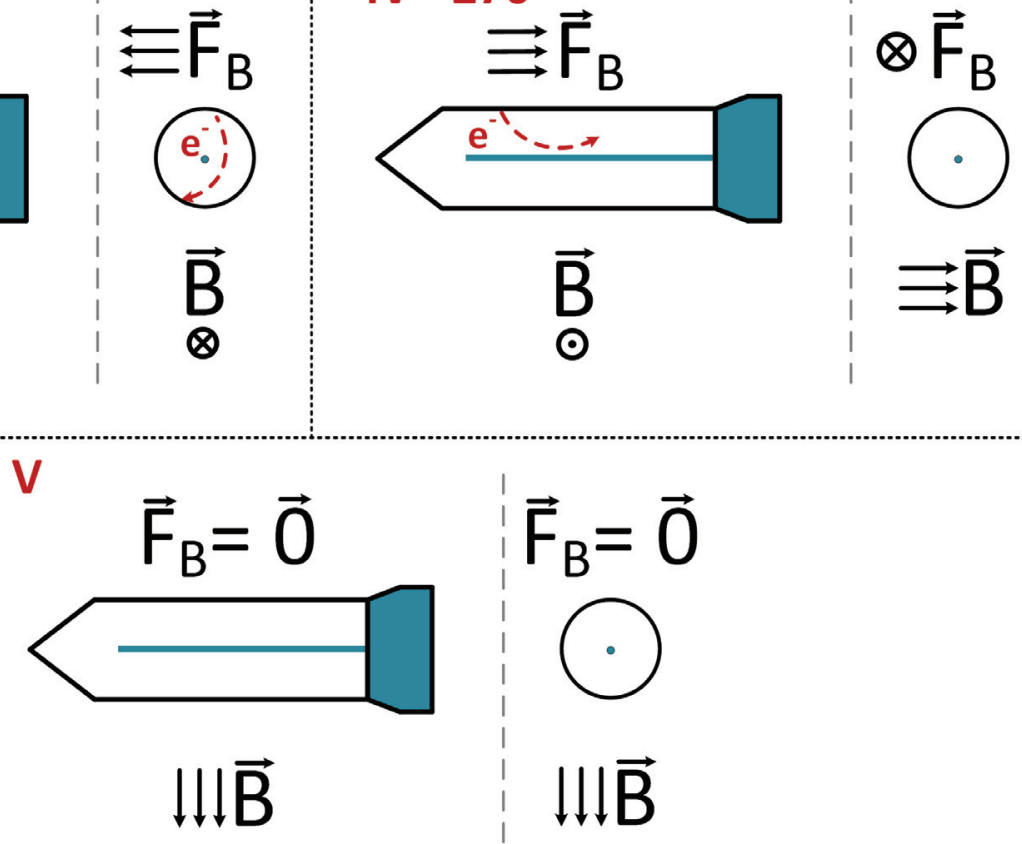

Figure 6.3: Influence of the magnetic field on electron trajectories at the four cardinal (I-IV) angles shown in Figure 6.2, as well as for the orientation where the magnetic field is parallel to the incoming photon beams. The photon beam is incoming from above in all images. On the left of each of the panels is a cross section along the length of the ion chamber, and the right panel is again a cross section but now with the chamber tip oriented out of the page. $\vec{F}_{B}$ represents the force due to the magnetic field on an idealized electron travelling down the page. The dashed lines inside the ion chambers are meant to demonstrate the overall effect of the magnetic field and represent a sample electron trajectory.

A variety of chamber sizes are included in this study. These are the Exradin A1SL, A12S, and A19, the PTW 31006 and 31010, and the NE 2571. Details on these chambers can be found in Fig. 6.4. The magnetic and photon field combinations that 
are used for these calculations are ${ }^{60} \mathrm{Co}$ spectrum ${ }^{74}$ at $0.35 \mathrm{~T}$ and a $7 \mathrm{MV}$ spectrum ${ }^{65}$ at $1.5 \mathrm{~T}$ (these are chosen based on two existing MRgRT machines). The beam-quality specification details for these photon beams are given later (Table 7.6).

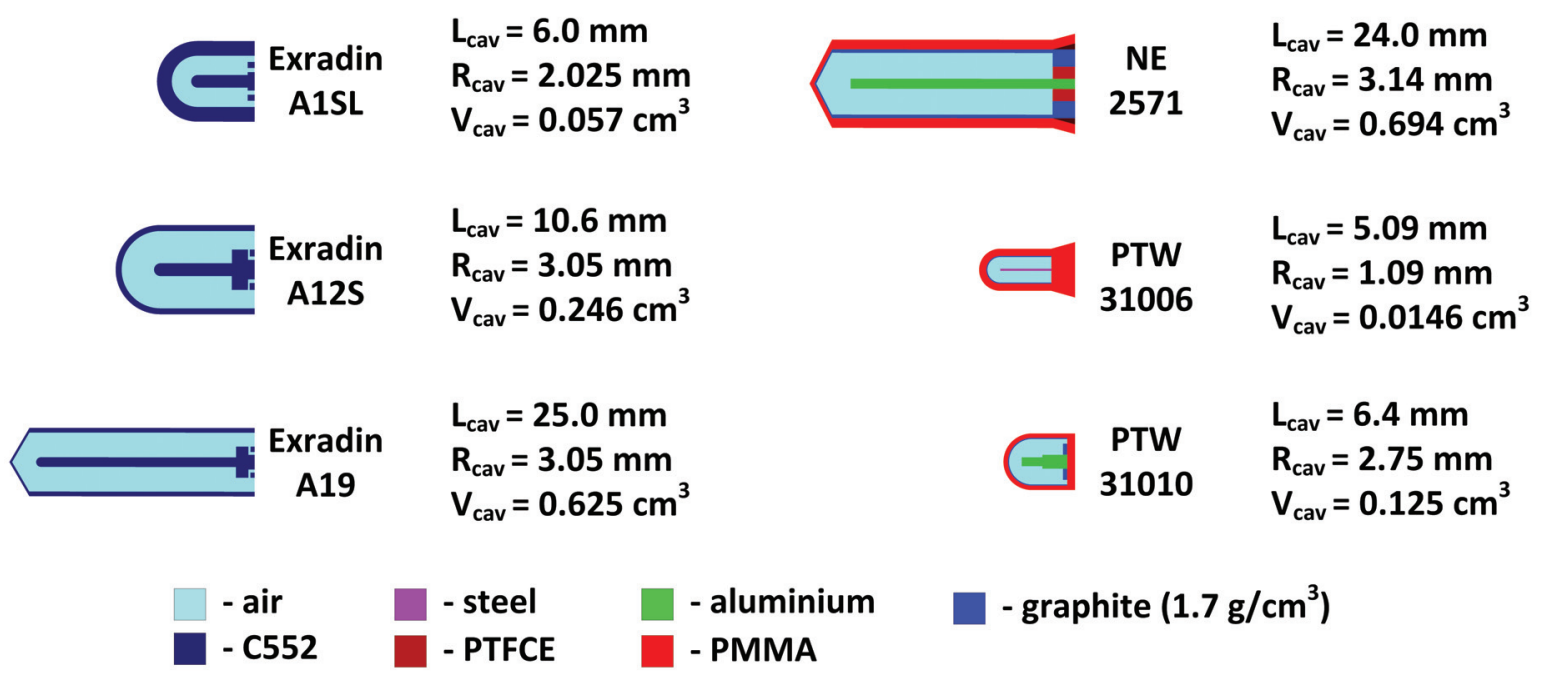

Figure 6.4: Ion chamber models used in calculating $\mathrm{k}_{Q}^{\text {mag }}$ as a function of angle. The scale between the chambers is similar to allow for visual comparison. The total cavity length, $\mathrm{L}_{\text {cav }}$, and radius, $\mathrm{R}_{\text {cav }}$, are given to the right of each chamber model. Details of stem are simulated, but omitted from the images. Material acronyms are for air equivalent plastic (C552), polymethyl methacrylate (PMMA), and polychlorotrifluoroethylene (PTFCE). The NE 2571 is simulated with a $1 \mathrm{~mm}$ PMMA waterproofing sleeve.

\subsubsection{Results and Discussion}

Fig. 6.5 provides $\mathrm{k}_{Q}^{m a g}$ as a function of angle for the six ion chambers and magnetic and photon field pairs. As a comparison, the $\mathrm{k}_{Q}(0 \mathrm{~T})$ values for each chamber are also given. There is a clear angular dependence of $\mathrm{k}_{Q}^{\text {mag }}$ for chambers and photon energies. The largest deviation, with the exception of the $\mathrm{NE} 2571$ from the $0 \mathrm{~T} \mathrm{k}_{Q}$ value is observed at C-IV, $270^{\circ}$, in which the electrons curve towards the chamber stem. The results for the NE 2571 do not correspond to the over $8 \%$ difference in ion chamber relative response as a function of angle measured by Smit et al. ${ }^{76}$ and are in much better agreement with the simulated data presented by O'Brien et al. ${ }^{26}$ which showed a roughly $4 \%$ change in

the chamber's relative response between C-I and C-II. The expected difference in $\mathrm{k}_{Q}^{\text {mag }}$ 
values mentioned in Sec. 6.1, when the electrons are bending toward the stem or the tip, is evident when comparing the results of C-II, 90 , and C-IV, 270 . Although at both of these orientations the magnetic field is perpendicular to the incoming photon field and the long axis of the chamber, differences in the geometric regions into which the electrons are swept produce dramatic changes in chamber dose. This is observed for all chambers, and is particularly highlighted for the ${ }^{60} \mathrm{Co}$ simulations of the Exradin A1SL, Exradin A12S, and the PTW 31006 in which the chamber dose, in comparison to the 0 T results, decreases near the C-II orientation and increases near the C-IV orientation. The C-I, $0^{\circ}$, and C-III, $180^{\circ}$, show a reduced overall effect due to the magnetic field as compared to C-II and C-IV. Further, the values of $\mathrm{k}_{Q}^{m a g}$ at C-I and C-III show no statistically significant differences in the Monte Carlo simulations since the chambers are cylindrically symmetric. This provides an advantage for ion chamber dosimetry in magnetic fields, as there would be a reduced ambiguity in chamber alignment if C-I and C-III are chosen as the recommended dosimetry orientations. Moreover, any asymmetry in the physical chambers would introduce some variability in the chamber relative response between these two orientations, and experimental studies are required to determine the magnitude of the effect

The dose-to-water, at $10 \mathrm{~cm}$ depth, compared to the $0 \mathrm{~T}$ simulation, for the case when the magnetic field is perpendicular to the incoming photon beam changes by $(-0.060 \pm 0.014) \%$ and $(-0.857 \pm 0.014) \%$ for the ${ }^{60} \mathrm{Co}(0.35 \mathrm{~T})$ and $7 \mathrm{MV}(1.5 \mathrm{~T})$ simulations, respectively. The $7 \mathrm{MV}(1.5 \mathrm{~T})$ water dose changes are slightly larger in magnitude than the $(-0.5 \pm 0.1) \%$ reported by O'Brien et al. ${ }^{26}$. For the case when the magnetic field is parallel to the incoming photon beam the dose-to-water changes by $(-0.007 \pm 0.014) \%$ and $(0.171 \pm 0.014) \%$ for the ${ }^{60} \mathrm{Co}(0.35 \mathrm{~T})$ and $7 \mathrm{MV}(1.5 \mathrm{~T})$ simulations, respectively. These changes are included in the $\mathrm{k}_{Q}^{\operatorname{mag}}$ and $\mathrm{k}_{B}$ calculations. 

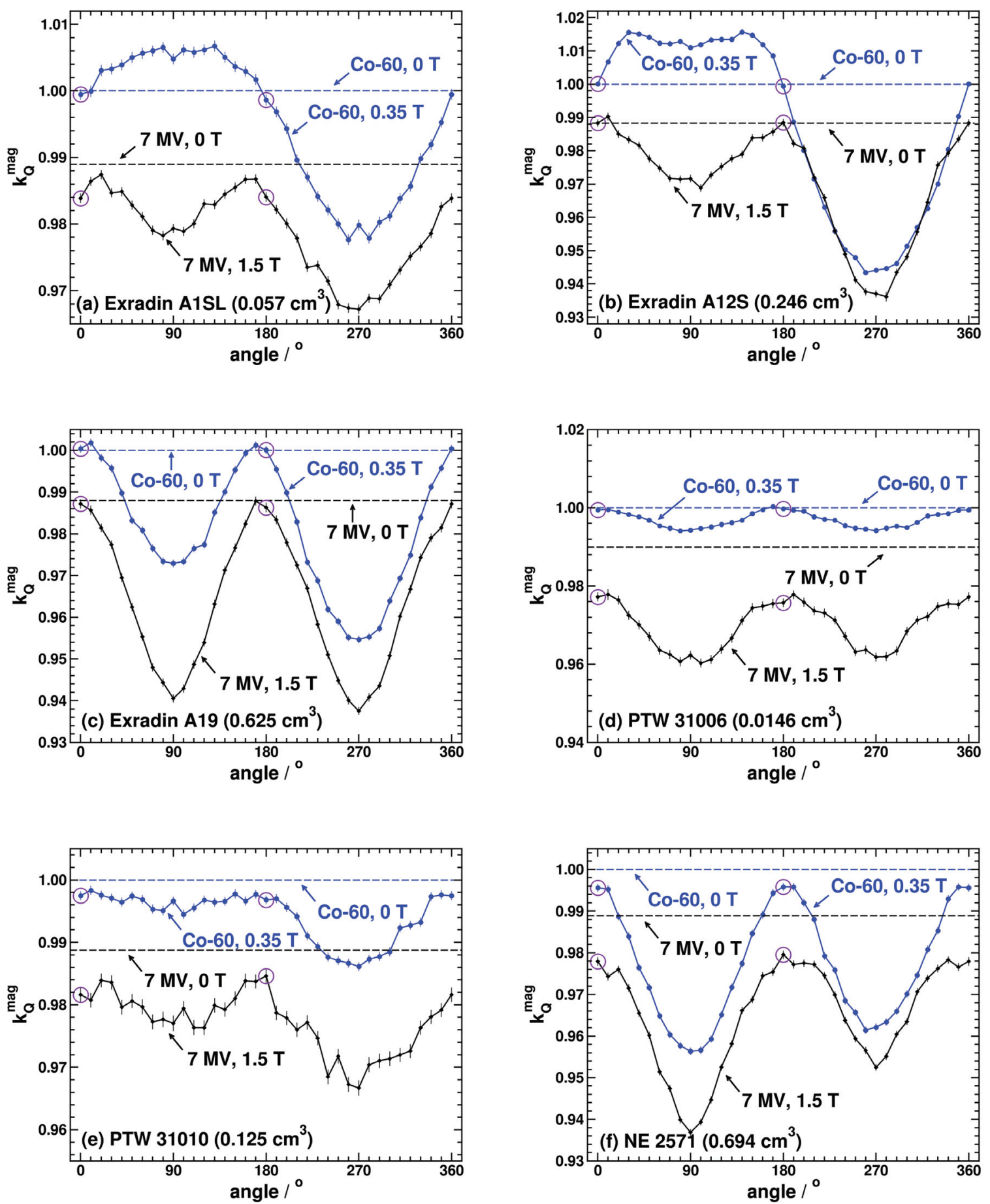

Figure 6.5: $\mathrm{k}_{Q}^{\mathrm{mag}}$ as a function of angle for ${ }^{60} \mathrm{Co}$ at $0.35 \mathrm{~T}$ and $7 \mathrm{MV}$ at $1.5 \mathrm{~T}$. The horizontal dashed lines are the $0 \mathrm{~T} \mathrm{k}_{Q}$ values for the chambers and associated beam quality $\left(\mathrm{k}_{Q}=1\right.$ for $\left.{ }^{60} \mathrm{Co}\right)$. Simulation uncertainty is $\mathrm{k}=1$. The $0 \mathrm{~T}$ results are for the $0^{\circ}$ simulation only (sample simulations demonstrate that there is no variation in $\mathrm{k}_{Q}$ as a function of angle in the absence of a magnetic field). The entire geometric sensitive volume is used. The circles at 0 and $180^{\circ}$ are for guiding the eye. 


\subsection{Sensitive volume effects as a function of angle}

As mentioned above, when a magnetic field is present the true size of the ion chamber's collection volume plays an important role in the simulated dose-to-air ${ }^{27,28}$. This is due to the charge collecting electric field of the chamber having a fringing effect near the stem and guard electrode, and a portion of "dead" air is formed from which the charge released is not collected ${ }^{70,71}$. In $\mathrm{C}$-II and $\mathrm{C}$-IV the effect of the sensitive volume is expected to be most noticeable since the influence of the magnetic field curves the electrons along the length of the chamber.

To investigate this effect, the air cavities of the Exradin A1SL and PTW 31010 are segmented into $0.1 \mathrm{~mm}$ segments along the length of the chambers, and the dose to each of these regions is determined as a function of distance along the chamber axis. This simulation uses the same geometric set-up described in Sec. 6.1. A ${ }^{60}$ Co photon beam is incident and the magnetic field is set to be either $0 \mathrm{~T}$ or $1 \mathrm{~T}$. For the $1 \mathrm{~T}$ simulation the five configurations from Figure 6.3 are simulated. The results are normalized by the average dose to the entire geometric sensitive volume at $0 \mathrm{~T}$.

To evaluate the effect of the sensitive volume on $\mathrm{k}_{Q}^{m a g}$, the geometric sensitive volume of each of the six chambers in Sec. 6.1 is segmented into two portions, as shown in Fig. 6.6. Section (1) corresponds to the volume of air associated with the first $1 \mathrm{~mm}$ of length away from the stem, and section (2) is the remaining geometric sensitive volume. $\mathrm{k}_{Q}^{m a g}$ is evaluated for each of the chambers using only section (2) as the sensitive volume and at the same magnetic and photon field pairs used in Sec. 6.1. These $\mathrm{k}_{Q}^{m a g}$ values are then compared to those obtained when using the entire geometric sensitive volume (regions (1) and (2) combined) which are the calculations described in Sec. 6.1. The size of the excluded volume is comparable to that determined in other studies $^{70,71}$. The goal in this work is to determine if there are optimal orientations in 
which variations in the collection volume do not play a major role in the MC calculated dose-to-air in the chamber, and, therefore, the full details of the true collection volume are not as important here.
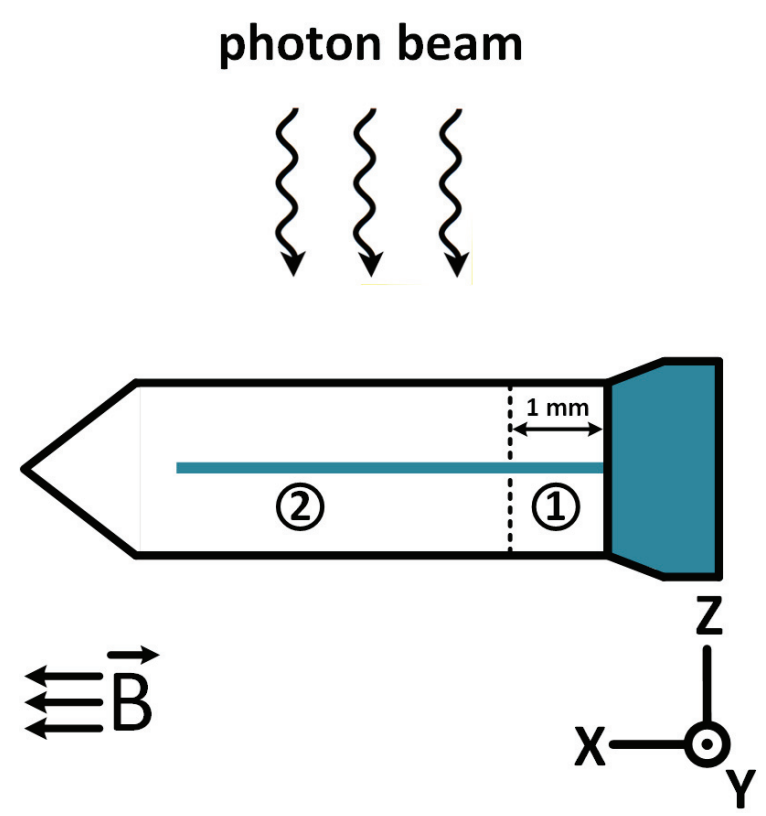

Figure 6.6: Cross section along the length of the ion chamber. Region (1) represents the air volume associated the first $1 \mathrm{~mm}$ away from the stem, and region (2) is the remainder of the geometric sensitive volume. Not to scale.

\subsubsection{Results and Discussion}

Fig. 6.7 provides the dose-to-air, normalized to the $0 \mathrm{~T}$ air cavity average, in $0.1 \mathrm{~mm}$ segments along the length of the Exradin A1SL and PTW 31010. The results are plotted with the start of the geometric sensitive volume near the stem being $0 \mathrm{~cm}$. The results here reflect the anticipated effect of the magnetic field described in Sec. 6.1. For the C-II simulation, there is an increase in the dose near the tip and a reduction near the stem of the chamber. The reverse effect is seen for the C-IV simulation, where the stem region sees a higher dose than the tip. This means that excluding the first $1 \mathrm{~mm}$ of air from the sensitive volume used for the MC calculation would increase the average chamber dose-to-air for the C-II simulation, while there would be a reduction 

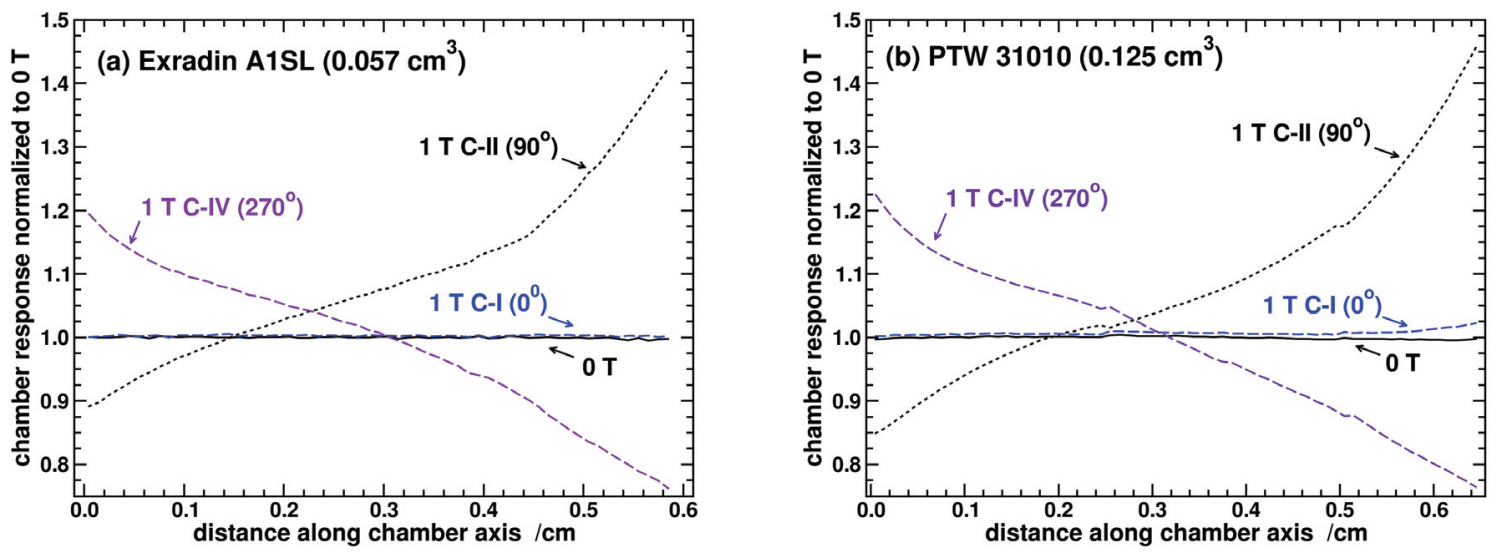

Figure 6.7: Dose per unit fluence, normalized to the average chamber dose at $0 \mathrm{~T}$, in the air volume corresponding to $0.1 \mathrm{~mm}$ intervals along the length of the Exradin A1SL and PTW 31010 in a 7 MV beam. Results for a 0 T magnetic field, and a $1 \mathrm{~T}$ magnetic field in the C-I, C-II, and C-IV orientations are shown. The C-V simulation results are omitted for clarity as they overlaps with the $0 \mathrm{~T}$ result. The normalization values are $2.7156 \times 10^{-12} \mathrm{~Gy} \mathrm{~cm}^{2}$ and $2.696 \times 10^{-12} \mathrm{~Gy} \mathrm{~cm}^{2}$ for the Exradin A1SL and PTW 31010, respectively.

for the C-IV simulation. This translates into a decrease in $\mathrm{k}_{Q}^{\text {mag }}$ for C-II and an increase for C-IV. The C-I simulation results very closely follow the $0 \mathrm{~T}$ results (as do the CII results - not shown). This is consistent with the results seen in Sec. 6.1.1 where the $\mathrm{C}-\mathrm{I}$ and $\mathrm{C}$-III orientations, on the whole, experience a minimal effect due to the magnetic field. Further, this indicates that the C-I and C-III orientations may reduce the importance of having the true sensitive volume in the $\mathrm{MC}$ simulation. The $\mathrm{C}-\mathrm{V}$ simulation produces little difference from the $0 \mathrm{~T}$ results since the magnetic force is fairly minimal for this orientation. For the C-II orientation at $1 \mathrm{~T}$, the larger NE 2571 shows similar results to those of the smaller chambers, with the main difference being a uniform dose region between roughly the first and last $0.7 \mathrm{~cm}$. Values of $\mathrm{k}_{Q}^{\text {mag }}$ as a function of angle calculated using the entire geometric sensitive volume (0 mm) or with region 1 (as shown in Fig. 6.6), excluded from the MC simulation (1 mm) are given in Fig. 6.8. There are evident differences between the $\mathrm{k}_{Q}^{\mathrm{mag}}$ values calculated using the different volumes and the effect is clearly ion chamber, photon energy, and magnetic 
field dependent. The largest impact is at $90^{\circ}$ and $270^{\circ}$, and is most pronounced for the Exradin A1SL and PTW 31010, two of the smaller chambers. The PTW 31006, the smallest chamber by volume, does not see much impact due to its reduced chamber radius. The larger volume chambers, Exradin A19 and NE 2571, also observe an impact due to the reduced sensitive volume, although to a lesser degree compared to the smaller chambers. On the whole, the ${ }^{60}$ Co simulations demonstrate a larger variation of $\mathrm{k}_{Q}^{\text {mag }}$ as the volume is reduced because at the lower-energy, compared to the $7 \mathrm{MV}$ beam, secondary electrons have a tighter radius of curvature in the magnetic field.

The results at $0^{\circ}$ and $180^{\circ}$ reflect the results of the simulations presented in Fig. 6.7. Due to the fairly uniform dose distribution in the chamber at these angles, a reduction in the sensitive volume does not produce an effect on the dose-to-air in the chamber and $\mathrm{k}_{Q}^{\text {mag }}$ is unaffected as well. This further indicates that the C-I and C-III orientations are optimal for ion chamber dosimetry in magnetic fields. 

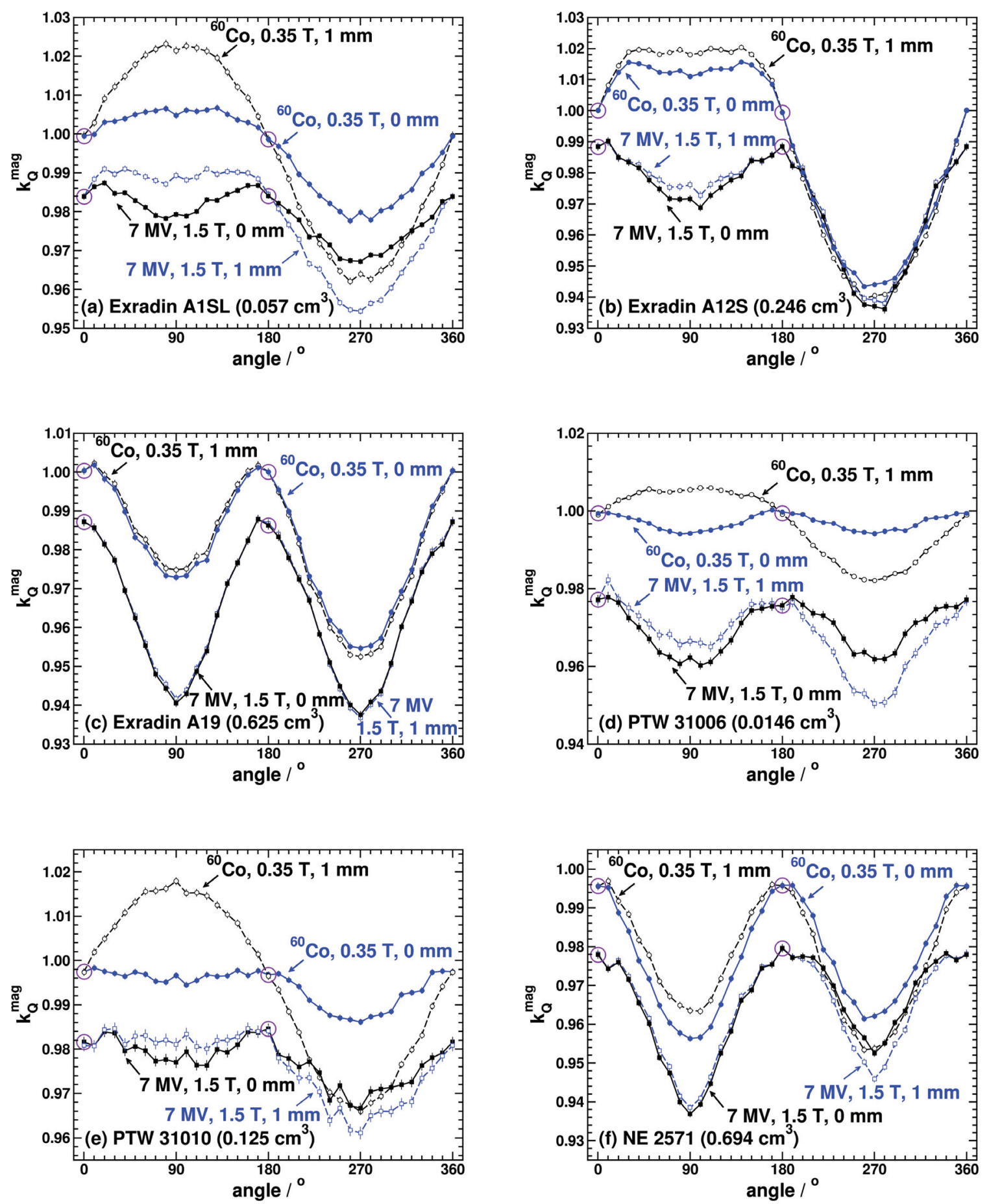

Figure 6.8: Values of $\mathrm{k}_{Q}^{\mathrm{mag}}$ as a function of angle for ${ }^{60} \mathrm{Co}$ at $0.35 \mathrm{~T}$ and $7 \mathrm{MV}$ at $1.5 \mathrm{~T}$ with either the full geometric sensitive volume $(0 \mathrm{~mm}$ labels $)$ or with the geometric sensitive volume reduced by the volume corresponding to $1 \mathrm{~mm}$ away from the stem (1 mm labels) used for the ion chamber simulations. The circles at 0 and $180^{\circ}$ are for guiding the eye. 


\subsection{Variations in $\mathbf{k}_{Q}^{\operatorname{mag}}$}

The angular calculations in the previous two section can be applied to determine the amount of variation that would occur due to chamber misalignments and to approximate the error that would be incurred at each of the four cardinal angles if the effect of the sensitive volume is completely neglected. This is important information for determining the uncertainty of clinical reference dosimetry measurements.

The change in $\mathrm{k}_{Q}^{m a g}$ due to orientation and sensitive volume changes are evaluated using the results from the sections above. $\Delta k_{Q}^{\text {mag }}(\theta)$ is defined as

$$
\Delta k_{Q}^{\operatorname{mag}}(\theta)=100 \% \times \frac{\max \left(\left|k_{Q}^{\operatorname{mag}}\left(\theta \pm 10^{\circ}\right)-k_{Q}^{m a g}(\theta)\right|\right)}{k_{Q}^{\operatorname{mag}}(\theta)} .
$$

It is the maximum magnitude percent difference between the $k_{Q}^{m a g}$ at a given angle, $\theta$, and the $k_{Q}^{m a g}$ associated with a $10^{\circ}$ misalignment either above $(+)$ or below $(-) \theta$. $\Delta k_{Q}^{m a g}(\theta, \Delta V)$ is defined as

$$
\Delta k_{Q}^{m a g}(\theta, \Delta V)=100 \% \times \frac{k_{Q}^{m a g}(\theta, 1 m m)-k_{Q}^{m a g}(\theta, 0 m m)}{k_{Q}^{m a g}(\theta, 0 m m)} .
$$

It corresponds to the percent difference in $\mathrm{k}_{Q}^{m a g}$ observed at angle $\theta$ between the simulation using the full geometric sensitive volume, $0 \mathrm{~mm}$, and that with the air volume corresponding to the first $1 \mathrm{~mm}$ away from the stem excluded. Both of these quantities are evaluated for the four cardinal angles and for the ${ }^{60} \mathrm{Co}(0.35 \mathrm{~T})$ and $7 \mathrm{MV}(1.5 \mathrm{~T})$ cases.

\subsubsection{Results and Discussion}

Table 6.1 provides $\Delta k_{Q}^{m a g}(\theta)$ and $\Delta k_{Q}^{\text {mag }}(\theta, \Delta V)$ values for the ${ }^{60}$ Co and $7 \mathrm{MV}$ simulations. The results are given for the $0^{\circ}, 90^{\circ}, 180^{\circ}$, and $270^{\circ}$ orientations, and for the $0 \mathrm{~mm}$ 
simulation for $\Delta k_{Q}^{m a g}(\theta)$. The maximum deviation observed for $\Delta k_{Q}^{\operatorname{mag}}(\theta)$ is $0.98 \%$ and $1.07 \%$ for the $7 \mathrm{MV}$ and ${ }^{60} \mathrm{Co}$ simulations, respectively. The 0 and $180^{\circ}$ orientations observe slightly larger $\Delta k_{Q}^{\text {mag }}(\theta)$ values than the $90^{\circ}$ and $270^{\circ}$ orientations. Overall, variation in the sensitive volume does not produce large differences in $\Delta k_{Q}^{\text {mag }}(\theta)$ for the 7 MV simulation, but can more than double $\Delta k_{Q}^{m a g}$ for the ${ }^{60}$ Co case for the 0 and $180^{\circ}$ orientations for the smaller volume chambers (not shown). Practically, this means that if an ion chamber measurement is made in the MRgRT set up with a $\pm 10^{\circ}$ tilt from the angle, $\theta$, for which the $\mathrm{k}_{Q}^{\text {mag }}$ is calculated, there is an error in the dose comparable to the $\Delta k_{Q}^{\text {mag }}(\theta)$ values given in Table 6.1.

Table 6.1: Values of $\Delta k_{Q}^{\text {mag }}(\theta)$, maximum absolute percent difference in $\mathrm{k}_{Q}^{\text {mag }}$ due to a $10^{\circ}$ chamber misalignment, and $\Delta k_{Q}^{m a g}(\theta, \Delta V)$, percent difference between $\mathrm{k}_{Q}^{\text {mag }}$ calculated using the full geometric sensitive volume and excluding the air corresponding to the first $1 \mathrm{~mm}$ away from the stem, for the $7 \mathrm{MV}$ and ${ }^{60} \mathrm{Co}$ simulations for the four cardinal angular orientations. The statistical uncertainty on each value is $\lesssim 0.15 \%$ (absolute error), and correlations may reduce uncertainty in some cases.

\begin{tabular}{|c|c|c|c|c|c|c|c|c|}
\hline \multirow[b]{2}{*}{$\theta$} & \multicolumn{4}{|c|}{$\Delta \bar{c}_{\Delta}^{\text {mag }}(\theta)(\%)$} & \multicolumn{4}{|c|}{$\Delta k_{Q}^{\text {mag }}(\theta, \Delta V)(\%)$} \\
\hline & $0^{\circ}$ & $90^{\circ}$ & $180^{\circ}$ & $270^{\circ}$ & $\mathbf{0}^{o}$ & $90^{\circ}$ & $180^{\circ}$ & $270^{\circ}$ \\
\hline & \multicolumn{8}{|c|}{$7 \mathrm{MV}$} \\
\hline Exradin A1SL & 0.26 & 0.11 & 0.27 & 0.17 & -0.01 & -1.02 & -0.05 & 1.32 \\
\hline Exradin A12S & 0.49 & 0.29 & 0.64 & 0.09 & -0.02 & -0.47 & 0.00 & -0.21 \\
\hline Exradin A19 & 0.58 & 0.40 & 0.30 & 0.35 & -0.02 & -0.12 & -0.05 & 0.08 \\
\hline PTW 31006 & 0.54 & 0.21 & 0.21 & 0.27 & 0.02 & -0.44 & 0.06 & 1.18 \\
\hline PTW 31010 & 0.25 & 0.24 & 0.60 & 0.38 & 0.08 & -0.41 & 0.05 & 0.58 \\
\hline \multirow[t]{2}{*}{ NE 2571} & 0.37 & 0.33 & 0.42 & 0.42 & -0.03 & -0.18 & -0.01 & 0.69 \\
\hline & \multicolumn{8}{|c|}{${ }^{60} \mathrm{Co}$} \\
\hline Exradin A1SL & 0.42 & 0.17 & 0.31 & 0.23 & -0.01 & -1.66 & -0.01 & 1.62 \\
\hline Exradin A12S & 0.97 & 0.19 & 1.07 & 0.07 & 0.00 & -0.70 & -0.01 & 0.39 \\
\hline Exradin A19 & 0.46 & 0.05 & 0.46 & 0.07 & 0.00 & -0.20 & 0.00 & 0.23 \\
\hline PTW 31006 & 0.24 & 0.05 & 0.25 & 0.06 & 0.04 & -1.13 & 0.06 & 1.21 \\
\hline PTW 31010 & 0.09 & 0.21 & 0.09 & 0.12 & 0.01 & -2.13 & 0.03 & 2.05 \\
\hline NE 2571 & 0.14 & 0.03 & 0.18 & 0.14 & 0.01 & -0.75 & 0.01 & 0.87 \\
\hline
\end{tabular}

A $10^{\circ}$ misalignment of the chamber is fairly noticeable to the eye, and these results are meant to be used as a guide for dosimetry measurements. Simulations with the PTW 31010 and the Exradin A19 chambers show that the variation in $\mathrm{k}_{Q}^{\text {mag }}$ due to 
a $3^{\circ}$ misalignment in the $\mathrm{x}-\mathrm{y}$ plane about the $0^{\circ}, 90^{\circ}, 180^{\circ}$, and $270^{\circ}$ orientations are less than $0.2 \%$ and $0.3 \%$ for the ${ }^{60} \mathrm{Co}$ and $7 \mathrm{MV}$ spectra, respectively. For a $3^{\circ}$ misalignment along the z-axis (i.e., tipping the chamber towards or away from the photon beam), the $0^{\circ}$ and $180^{\circ}$ orientations showed less than $0.2 \%$ and $0.4 \%$ variation for the ${ }^{60} \mathrm{Co}$ and $7 \mathrm{MV}$ spectra, respectively, for both chambers, while the $90^{\circ}$ and $270^{\circ}$ orientations produced differences as large as $1.4 \%$ and $1.0 \%$ for the ${ }^{60} \mathrm{Co}$ and 7 MV spectra, respectively. The statistical uncertainty on all of these difference is about $0.14 \%$. These results are in agreement with the findings of Reynolds et al. ${ }^{7}$, in which a $3^{\circ}$ misalignment in any direction about the the $90^{\circ}$ and $270^{\circ}$ orientations produced a maximum variation in dose of roughly $1 \%$ and an average variation of about $0.5 \%$. Further, although slightly larger $\Delta k_{Q}^{m a g}(\theta)$ values are observed for $0^{\circ}$ and $180^{\circ}$ in Table 6.1, the advantage of nearly completely eliminating the effect of the sensitive volume on $\mathrm{k}_{Q}^{\mathrm{mag}}$, as compared to the $90^{\circ}$ and $270^{\circ}$ simulations, and the absence of a difference between the $\mathrm{k}_{Q}^{m a g}$ values at 0 and $180^{\circ}$, as described in Sec. 6.1.1, makes the C-I and C-III orientations the ideal orientations for ion chamber dosimetry in magnetic fields. Additionally, as is seen in Table 6.1's $\Delta k_{Q}^{\operatorname{mag}}(\theta, \Delta V)$ results, in these orientations the lack of variation in $\mathrm{k}_{Q}^{\text {mag }}$ demonstrates that the details of the sensitive volume are negligible (as compared to the several percent change due to sensitive volume changes in the $\mathrm{C}-\mathrm{II}$ and $\mathrm{C}-\mathrm{IV}$ orientations).

\subsection{Summary}

As shown in chapter 5 , the unknown sensitive volume of the ion chamber poses a challenge to ion chamber dosimetry. Without a full description a several percent difference

between the true and calculated $\mathrm{k}_{Q}^{\text {mag }}$ would occur. By orienting the chamber to be parallel with the magnetic field for MRgRT machines which have the magnetic field perpendicular to the incoming photon beam, the effect of the magnetic field on the 
chamber dose can be reduced and the issue of the unknown sensitive volume mitigated. This is an important problem to overcome and sets the path for calculating ion chamber and beam quality specific magnetic field correction factors, as is done in the next chapter. Further, the variation in $\mathrm{k}_{Q}^{\text {mag }}$ due to a misalignment along the x-y plane, discussed in Section 6.3, is slightly increased at the optimal $0^{\circ}$ and $180^{\circ}$ orientations as compared to the $90^{\circ}$ and $270^{\circ}$ orientations, but the elimination of the sensitive volume problem and the reduced effect of the magnetic field at the $0^{\circ}$ and $180^{\circ}$ orientations make these the ideal candidates for magnetic field dosimetry. 


\section{Chapter 7}

\section{Ionization chamber magnetic field correction factors and additional considerations of mag- netic field dosimetry}

In this chapter the magnetic field correction factors for 32 cylindrical and three parallelplate ionization chambers are evaluated. The beam quality dependence of the $k_{B}$ and $k_{Q}^{m a g}$ values is determined, and the influence of the magnetic field on the beam-quality specifiers, TPR $R_{10}^{20}$ and $\% d d(10)_{x}$, is also considered. The $C$-I to $C$-V notation is maintained from chapter 6 . These are the results of the peer-reviewed paper III.

\subsection{Cylindrical ion chamber models}

For the $\mathrm{k}_{Q}, \mathrm{k}_{Q}^{m a g}$, and $\mathrm{k}_{B}$ calculations in this chapter all chambers previously described by Muir and Rogers ${ }^{69}$ are used. The only differences between the chambers in Muir and Rogers and the ones simulated here are in the PTW 30010/11/12/13, Exradin A14, T14, A14SL and A16, and the CC01. For the PTW chambers, blueprints from the manufacturer were used to give more detailed models of the chambers. The basic dimensions of the chambers remain equivalent to those described in Muir and Rogers, but the details of stem region better reflect the true geometry of the chambers. The Exradin chambers are simulated with an electrode composed of three individual layers 
of silver, copper, and steel instead of using a homogeneous composition for the entire electrode. The CC01 chamber, uses a slightly thinner electrode which reflects the dimensions provided in the blueprints from the manufacturer. These differences may reflect slight changes in the $\mathrm{k}_{Q}$ values obtained in this study when comparing to the TG-51 addendum values ${ }^{23}$.

In chapter 3, the magnetic field transport code was benchmarked via the Fano cavity test and comparison to experimental work. Experimental variables such as beam orientation and the unknown sensitive volume have been shown to cause discrepancies between measurements and Monte Carlo models, particularly at a magnetic field strengths higher than $1 \mathrm{~T}$, as seen in chapter 3 and 5 , and other works ${ }^{27-29,42}$. By varying the size of the sensitive volume good agreement between ion chamber relative response as a function of magnetic field was shown. The PTW 30013 stood out as $1.73 \%$ below the experimental value at $1.5 \mathrm{~T}$, and reducing the sensitive volume by the air volume corresponding to the first $1 \mathrm{~mm}$ away from the stem reduced this difference to $1.3 \%$. Recalculating with the updated blueprint based model used in this chapter and a $1.5 \mathrm{~mm}$ long "dead" volume region reduces the difference between the simulation and experiment to $0.35 \%$. Spindeldreier et al. ${ }^{27}$ compared experimental measurements with EGSnrc simulations (using a different magnetic field code) to show that, by adjusting the size of the sensitive volume used in the simulation, agreement with a root-meansquare deviation of $0.2 \%$ and a maximum difference of $0.9 \%$ could be achieved for the six chambers in their study. The experimental set up in Spindeldreier et al. had a PTW 30013 ion chamber oriented along the negative y-axis in a water phantom, and the photon beam was incoming along the z-axis. The magnetic field, which was directed in either the positive or negative $\mathrm{x}$-axis (corresponding respectively to $\mathrm{C}$-IV and C-II), was set to be perpendicular to both the photon beam and the long axis of the chamber. Using the updated PTW 30013 model and the same $1.5 \mathrm{~mm}$ "dead" volume region, our simulations observe a root-mean-square deviation of $0.2 \%$ and a maximum difference 
of $0.55 \%$ between the simulation and experimental relative response as a function of magnetic field from Spindeldreier et al. for this chamber. The results of this simulation and the experimental values can be seen in Fig. 7.1. Overall, this demonstrates that the unknown sensitive volume introduces a free parameter into these simulations, and differences which could be caused by the chamber model, transport mechanism, and other experimental factors can be masked by varying the size of the sensitive region. These variations in the sensitive volume do not necessarily reflect the size and shape of the true collection volume, and experimental measurements are necessary to verify the correction values given in this work and other studies.

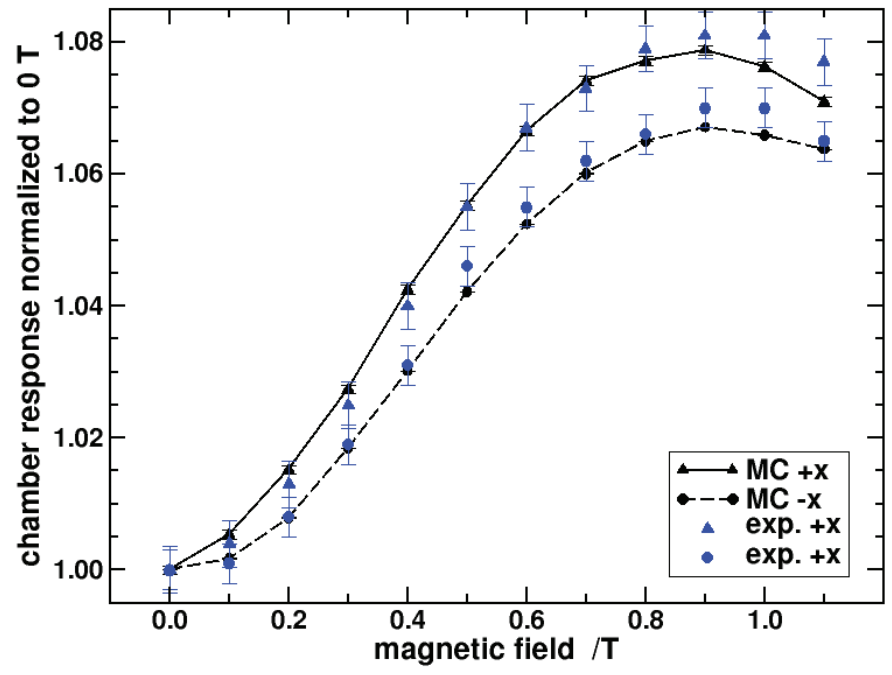

Figure 7.1: EGSnrc simulation comparison to experimental results from Spindeldreier et al. for the updated PTW 30013 model with a $1.5 \mathrm{~mm}$ "dead" region. The -x (C-II) and $+\mathrm{x}(\mathrm{C}-\mathrm{IV})$ labels indicate the direction of the magnetic field.

\subsection{Beam-quality dependence of $\mathbf{k}_{Q}^{\operatorname{mag}}$ and $\mathbf{k}_{B}$}

Because $\mathrm{k}_{Q}^{\text {mag }}$ corrects for both the presence of the magnetic field and the change in beam quality from ${ }^{60} \mathrm{Co}$ there is an explicit beam quality dependence included in this factor, much like the regular definition of $\mathrm{k}_{Q}$. Since $\mathrm{k}_{B}$ accounts only for the presence of the magnetic field, a reduced dependence on beam quality is anticipated. 
Both $\mathrm{k}_{Q}^{\text {mag }}$ and $\mathrm{k}_{B}$ are evaluated for the Exradin A1SL, A12S, A19, and NE 2571 for four beam qualities. The four beams are the Varian TrueBeam (6 MV, FFF), the Elekta MRgRT (7 MV), and Varian Clinical (6 MV and $10 \mathrm{MV}$ ). The beam-quality specifiers for these beams are found below in Table 7.6. It should be noted the $7 \mathrm{MV}$ MRgRT photon beam is flattening filter free (FFF), however the use of a thick aluminium shield hardens the beam and the spectrum appears to be close to a clinical filtered photon beam. The magnetic field, with a magnitude of $1.5 \mathrm{~T}$, is set to be either parallel to the chamber, $\|_{c h}$, or parallel to the incoming photon beam, $\|_{p h}$. The $\|_{c h}$ orientation is equivalent to $\mathrm{C}-\mathrm{I}$ and $\mathrm{C}-\mathrm{III}$, and $\|_{p h}$ is equivalent to $\mathrm{C}-\mathrm{V}$ (either in the positive or negative z-direction). This notation is introduced to remove any ambiguity regarding the direction of the magnetic field, and to facilitate the look-up of correction factors.

For a $0.35 \mathrm{~T}$ magnetic field, the beam quality dependence of $\mathrm{k}_{B}$ is evaluated for

the same four chambers in the $\|_{c h}$ and $\|_{p h}$ orientations. The ${ }^{60}$ Co, Varian TrueBeam (6MV, FFF), and Varian Clinical (6 MV and $10 \mathrm{MV}$ ) photon spectra are used. These simulations are aimed at determining if $\mathrm{k}_{B}$ correction factors calculated for a $0.35 \mathrm{~T}$ magnetic field and a ${ }^{60} \mathrm{Co}$ beam are applicable to the same magnetic field but for a roughly $6 \mathrm{MV}$ linac photon beam source (to work with the linac-based versions of the 0.35 T ViewRay MRgRT machine).

\subsubsection{Results and Discussion}

Having demonstrated that the C-I and C-III orientations are ideal for magnetic field dosimetry in the case when the magnetic field is perpendicular to the incoming photon beam, all subsequent results will deal exclusively with this orientation and the notation $\|_{c h}$ (i.e., C-I or C-III) is now applied to this alignment. For the case when the magnetic field is parallel with the photon beam, C-V, the notation $\|_{p h}$ will used.

As in the case of $\mathrm{k}_{Q}, \mathrm{k}_{Q}^{m a g}$ carries an explicit beam quality dependence since this 
factor adjusts for both the magnetic field's effects and the change in beam quality from the reference ${ }^{60}$ Co. Fig. 7.2 is a plot of $\mathrm{k}_{Q}^{\text {mag }}$ and $\mathrm{k}_{Q}$ as a function of the $0 \mathrm{~T}$ $\% \operatorname{dd}(10)_{x}$ beam-quality specifier. The first point, i.e., for the lowest $\% \operatorname{dd}(10)_{x}$, is for the Varian TrueBeam $6 \mathrm{MV}$ (FFF) spectrum, and the third point is for the $7 \mathrm{MV}$ MRgRT spectrum. The TG-51 addendum ${ }^{23}$ fits for each of the chambers are also plotted. Excluding the first point in all the graphs, for all of the chambers there is good agreement $\left(<0.2 \%\right.$ difference) between the simulated and TG-51 calculated $\mathrm{k}_{Q}$ values. The largest deviations are seen for the Exradin A12S chamber, for which the statistical uncertainty of the calculation and the uncertainty on the fit (which is performed over a much larger range of $\% \mathrm{dd}(10)_{x}$ values) can account for the differences. For the Varian TrueBeam, the maximum percent difference between the TG-51 calculated and the simulated $\mathrm{k}_{Q}$ values is $0.30 \%$. This is because the Varian TrueBeam is an FFF spectrum and the TG-51 addendum fits are determined based on flattened spectra ${ }^{69}$. These results are consistent with the variations in calculated and predicted stopping-powers for FFF beams seen by Dalaryd et al. ${ }^{78}$ In the TG-51 addendum, the provided formulas are said to be valid up to $0.2 \%$ deviation when applied to FFF spectra, but the results in this work indicate that the margin of uncertainty for applying the fit value for FFF beams should be increased to $0.3 \%$.

In these plots, for the Exradin chambers, it can be seen that $\mathrm{k}_{Q}^{\text {mag }}$ very closely follows the beam quality dependence of $\mathrm{k}_{Q}$. The NE 2571 chamber appears to have an additional beam quality dependence that is separate from that observed in $\mathrm{k}_{Q}$. To extract the beam quality dependence introduced by the magnetic field, $\mathrm{k}_{B}$ is calculated using Eq. 1.12.

Values of $\mathrm{k}_{B}$ as a function of $\% \mathrm{dd}(10)_{x}$ are given in Fig. 7.3. The Exradin chambers, the walls and electrode of which are composed of an air-equivalent plastic, C552, carry little beam quality dependence in the $\mathrm{k}_{B}$ correction factor. The percent difference are between the maximum and minimum $\mathrm{k}_{B}$ value, for the $\|_{c h}$ orientation, is $(0.18 \pm 0.15) \%$, 

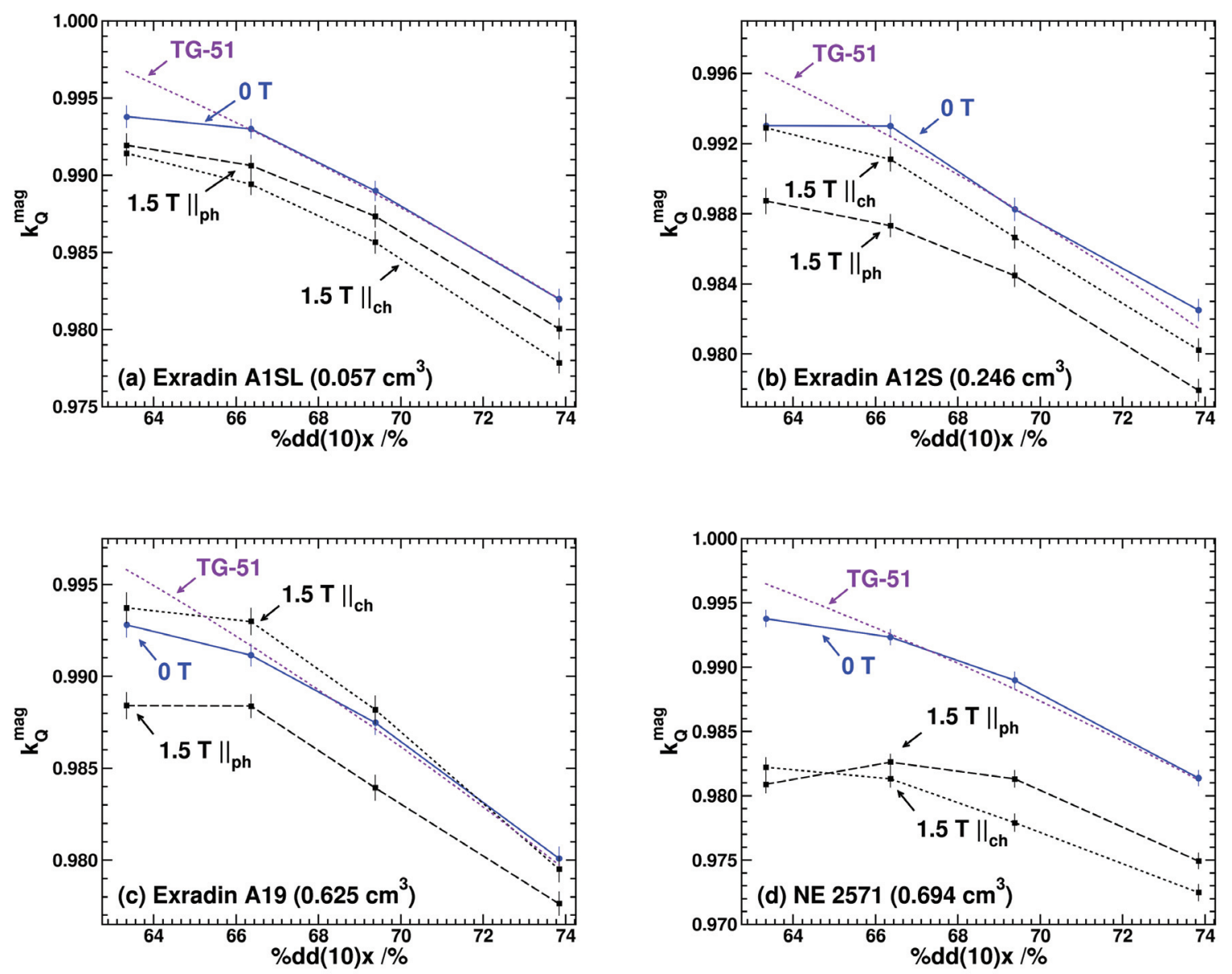

Figure 7.2: Values of $\mathrm{k}_{Q}^{m a g}$ and $\mathrm{k}_{Q}$ are for the $0 \mathrm{~T}$ magnetic field, and with a $1.5 \mathrm{~T}$ magnetic field oriented either parallel to the incoming photon beam, $\|_{p h}(\mathrm{C}-\mathrm{V})$, or parallel to the length of the chamber, $\|_{c h}(\mathrm{C}-\mathrm{I})$. The dashed lines without symbols are the TG-51 addendum ${ }^{23}$ fits for $\mathrm{k}_{Q}$ for each of the chambers. The Varian TrueBeam $6 \mathrm{MV}$ (FFF), $7 \mathrm{MV}$ (MRgRT), and Varian Clinical $6 \mathrm{MV}$ and $10 \mathrm{MV}$ photon spectra are used with the $\% \mathrm{dd}(10)_{x}$ as in Table 7.6.

$(0.22 \pm 0.15) \%,(0.25 \pm 0.15) \%$, and $(0.26 \pm 0.15) \%$ for the Exradin A1SL, Exradin A12S, Exradin A19, and NE 2571, respectively. For the $\|_{p h}$ orientation, the percent differences are $(0.11 \pm 0.15) \%,(0.07 \pm 0.15) \%,(0.19 \pm 0.15) \%$, and $(0.64 \pm 0.15) \%$ for the same chamber order. The PTW 31010 and PTW 30013, which also have high density electrodes, have a respective maximum to minimum percent difference of $(0.07 \pm 0.15) \%$ and $(0.11 \pm 0.15) \%$, for $\|_{c h}$, and $(0.32 \pm 0.15) \%$ and $(0.50 \pm 0.15) \%$, for $\|_{p h}$. These variations are similar to those cited by Spindeldreier et al. ${ }^{27}$. 
The $\mathrm{k}_{B}$ factor, expectedly, has a reduced beam quality dependence compared to $\mathrm{k}_{Q}^{m a g}$. Chambers with high-density electrodes appear to have a larger beam quality dependence in the $\|_{p h}$ orientation, and application of magnetic field correction factors for these chamber outside of the beam qualities provided in this report is not advised. For chambers with low-density electrodes, the use of $\mathrm{k}_{B}$ in combination with $\mathrm{k}_{Q}$ may the preferred choice over using the $\mathrm{k}_{Q}^{\text {mag }}$ factor since the TG-51 addendum functional fits can be used to account for variations in beam quality.
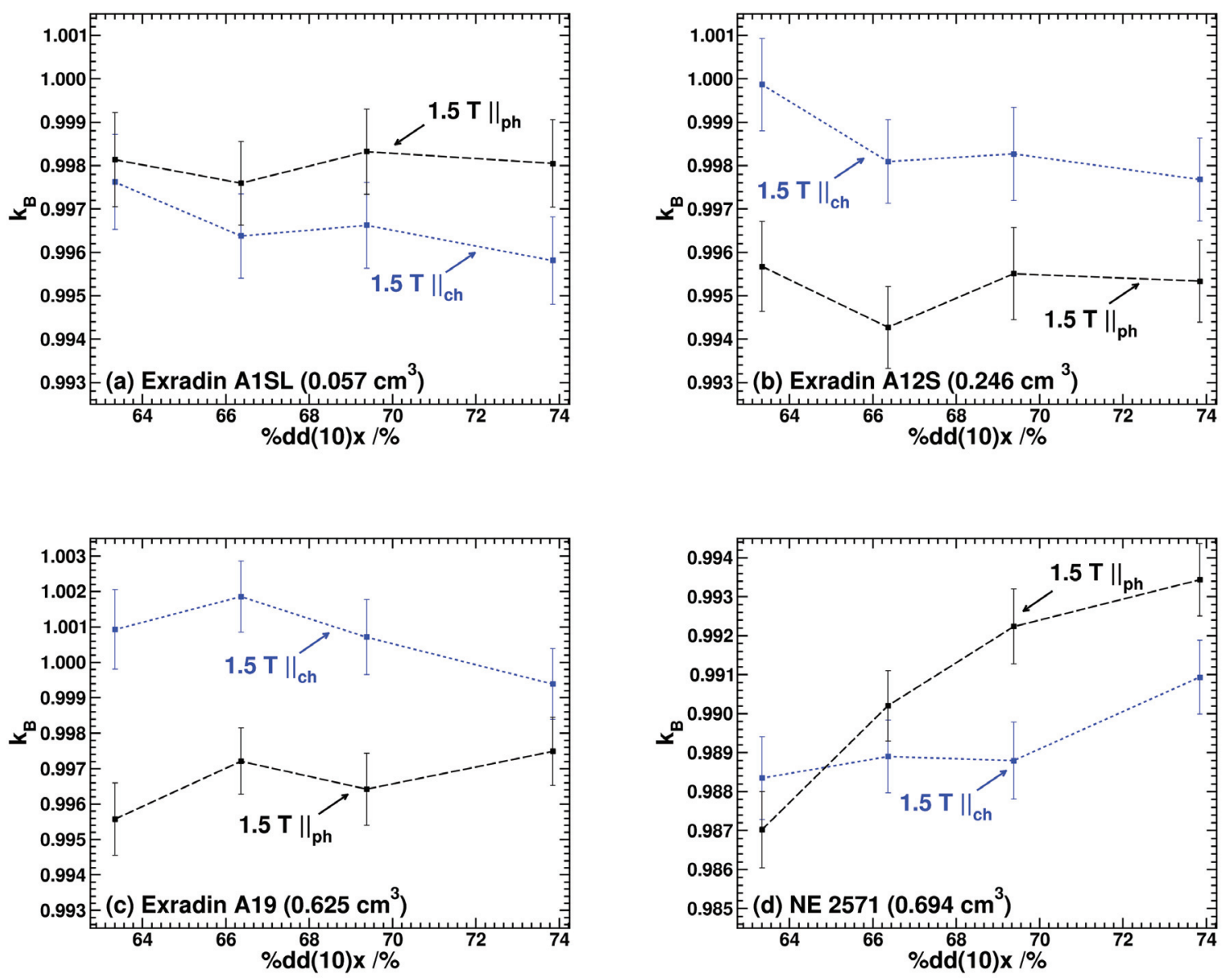

Figure 7.3: Results are as in Figure 7.2 but for $\mathrm{k}_{B}$.

Fig. 7.4 provides $\mathrm{k}_{B}$ as a function of $\% \mathrm{dd}(10)_{x}$ for the same four chambers but with a $0.35 \mathrm{~T}$ magnetic field. Percent differences between the maximum and minimum values for all the chambers in both the $\|_{p h}$ and $\|_{c h}$ orientations are less than $0.2 \%$ 
(0.1\% uncertainty, $\mathrm{k}=1)$. This demonstrates that $\mathrm{k}_{B}$ correction values calculated with a ${ }^{60} \mathrm{Co}$ beam and a $0.35 \mathrm{~T}$ magnetic field are applicable to higher-energy accelerator photon beam qualities.
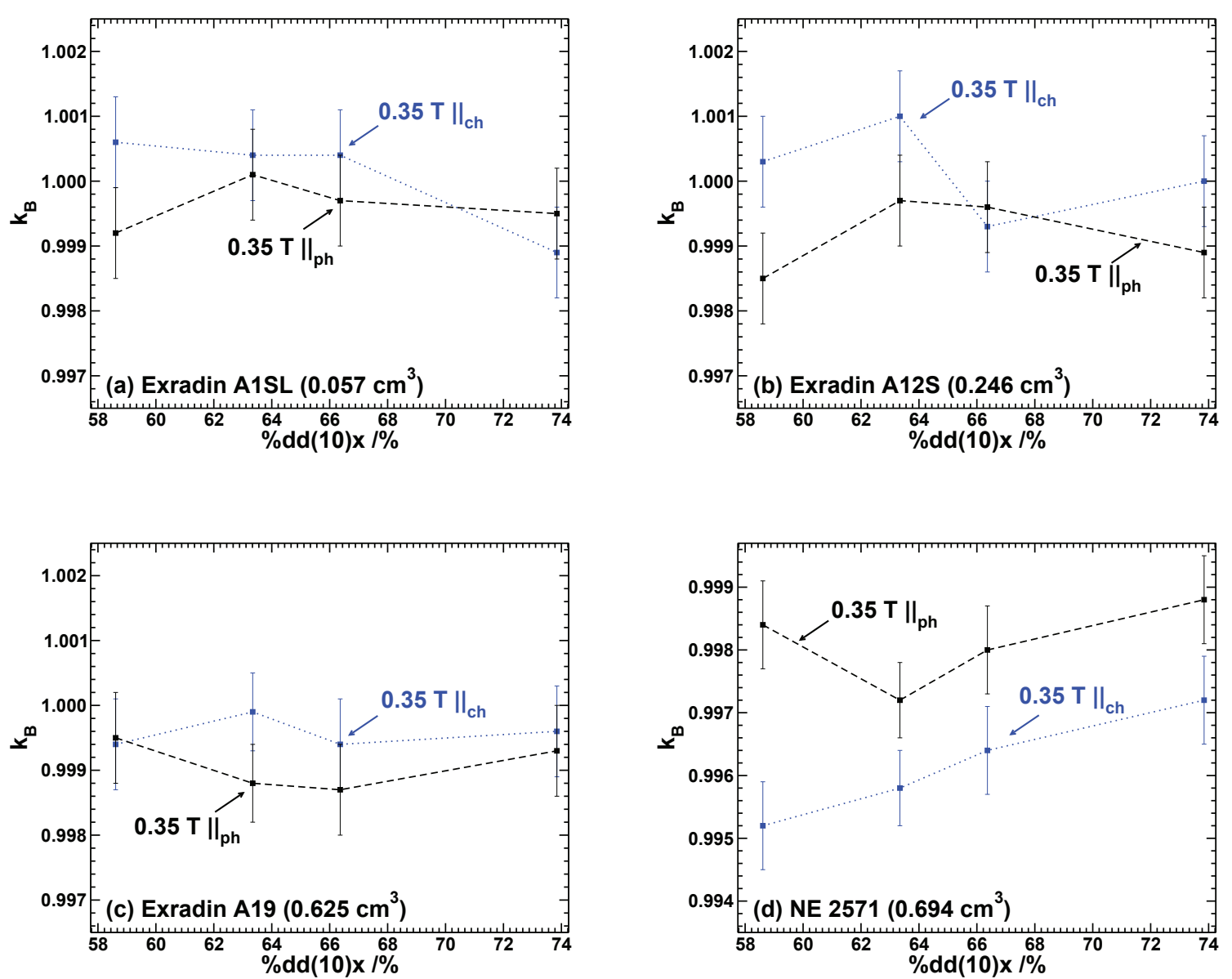

Figure 7.4: Results are as in Figure 7.3 but for a $0.35 \mathrm{~T}$ magnetic field and ${ }^{60} \mathrm{Co}$, Varian TrueBeam $6 \mathrm{MV}$ (FFF), and Varian Clinical $6 \mathrm{MV}$ and $10 \mathrm{MV}$ photon beams as per Table 7.6.

\section{3 $\mathbf{k}_{Q}^{m a g}$ and $\mathbf{k}_{B}$ calculations for ion chambers}

Having determined the optimal orientation of the ion chamber with respect to the magnetic field for the case when the magnetic field is parallel to the photon beam, the magnetic field correction factors can now be determined for a specific orientation. Using 
the geometry described in Sec. $6.1, \mathrm{k}_{Q}, \mathrm{k}_{Q}^{m a g}$, and $\mathrm{k}_{B}$ are evaluated for the chambers described by Muir and Rogers ${ }^{69}$. The reference ${ }^{60}$ Co $(0 \mathrm{~T})$ simulations are performed at $5 \mathrm{~cm}$ depth. The change in $\mathrm{k}_{Q}$ when using $10 \mathrm{~cm}$ depth for the ${ }^{60} \mathrm{Co}$ reference conditions is found to be less than $0.2 \%$ for the majority of chamber except for: (a) the CC01, which has a $0.47 \%$ increase in $\mathrm{k}_{Q}$ as compared to using $5 \mathrm{~cm}$ depth: and (b) for those chambers with an SPC electrode which caused up to a $0.79 \%$ increase. The calculations are performed with the magnetic field parallel to the chamber, $\|_{c h}$, or parallel to the incoming photon beam, $\|_{p h} . \mathrm{k}_{Q}$ is determined without any magnetic field. The correction factors are determined for a ${ }^{60} \mathrm{Co}$ beam at $0.35 \mathrm{~T}$ and the MRgRT $7 \mathrm{MV}$ photon beam at $1.5 \mathrm{~T}$.

The calculations performed in this section, as in the other sections, make use of the ICRU-37 ${ }^{79}$ data for stopping power calculations. By using the ICRU-37 data, a direct comparison of $\mathrm{k}_{Q}$ values can be made to the TG-51 addendum values ${ }^{23}$, and, as is shown below, the changes in $\mathrm{k}_{Q}$ due to using the ICRU-90 ${ }^{80}$ recommendation are within the statistical uncertainties in $\mathrm{k}_{Q}$.

\subsubsection{Results and Discussion}

Table 7.1 contains $\mathrm{k}_{Q}, \mathrm{k}_{Q}^{\text {mag }}$, and $\mathrm{k}_{B}$ values for ${ }^{60}$ Co beams at $0.35 \mathrm{~T}$ and $7 \mathrm{MV}$ beams at 1.5 T. For all of the chambers, with the exception of the PTW 30010/11/12/13, Exradin A14, T14, A14SL and A16, and CC01, there is very good agreement, within the statistical uncertainties, between the simulated $\mathrm{k}_{Q}$ values and those obtained from the fitted $\mathrm{k}_{Q}$ values. The TG-51 fits are used for the PTW 30010/11/12/13, and the fits from Muir and Rogers ${ }^{69}$ are applied for the Exradin A14, T14, A14SL and A16, and CC01, since these latter chambers are not recommended to be used for clinical reference dosimetry in the TG-51 addendum. For the PTW 30010/11/12/13, for which the MC models are now based on manufacturer provided blueprints as discussed in Sec. 7.1, the 
largest percent difference of $(0.38 \pm 0.14) \%$ is observed between the TG-51 fit and the simulated $\mathrm{k}_{Q}$ for the $7 \mathrm{MV}$ beam $(0 \mathrm{~T})$ for the PTW 30013. The simulated $\mathrm{k}_{Q}$ values for these chambers in this work are lower than those provided by the TG-51 fit, and the $\mathrm{k}_{Q}$ values for these PTW chambers produce better agreement with the experimental results in Muir et al. ${ }^{73}$. The Exradin A14, T14, A14SL and A16 models, which are made up of layers of steel-copper-silver instead of a homogeneous electrode, produced percent differences from the TG-51 addendum values of as low as $(-0.028 \pm 0.14) \%$ for the A15SL, and as large as $(-0.40 \pm 0.14) \%$, for the A16. A sample simulation for the A16 with a homogeneous electrode reduced the difference to below $0.2 \%$ (with the fit in Muir and Rogers ${ }^{69}$ having an uncertainty of $0.2 \%$ ). In Muir et al. the layered electrode model (private communication with Bryan Muir, 2017) produced good agreement with experimentally determined $\mathrm{k}_{Q}$ values. The CC01, modelled with a slightly thinner electrode than that used to determine the Muir and Rogers ${ }^{69}$ fit, produced a percent difference of $(-0.27 \pm 0.14) \%$ between the $\mathrm{k}_{Q}$ values calculated in this work and that of the Muir and Rogers ${ }^{69}$ fit.

For the ${ }^{60} \mathrm{Co}$ simulations at $0.35 \mathrm{~T}, \mathrm{k}_{B}$ correction factors are all within $0.81 \%$ and $0.61 \%$ of unity for $\|_{c h}$ and $\|_{p h}$, respectively. The most notable corrections, i.e. $>0.5 \%$, are seen for chambers that have a higher-Z (aluminium, steel, or steel-coppersilver) electrode, for example, the Exradin A14, Exradin A16, PTW 30013, FC65-P/G, and the NE 2571. For the $7 \mathrm{MV}$ simulations at $1.5 \mathrm{~T}$, the $\mathrm{k}_{B}$ correction factor is within $1 \%$ of 1.0 for the majority of chambers. Deviations larger than $1 \%$ from 1.0 in the $\mathrm{k}_{B}$ factor is associated with chambers with higher-Z electrodes. 
Table 7.1: Ion chamber $\mathrm{k}_{Q}, \mathrm{k}_{B}$, and $\mathrm{k}_{Q}^{m a g}$ values for ${ }^{60} \mathrm{Co}$ and $7 \mathrm{MV}$ photon beams. $\mathrm{k}_{Q}$ for ${ }^{60} \mathrm{Co}$ is 1 by definition (excluded). Statistical uncertainties $(\mathrm{k}=1)$ on all values are $\lesssim 0.1 \%$. Results are for a magnetic field either parallel to the photon beam, $\|_{p h}$, and perpendicular to the chamber or parallel to the length of the chamber, $\|_{c h}$, and perpendicular to the photon beam. Bold chambers are recommended by TG-51 addendum ${ }^{23}$ for reference dosimetry. Bold $\mathrm{k}_{B}$ values are $<0.99$, and italicised are between 0.99 and 0.995, and all others are $>0.995$. ( $\left.{ }^{*}\right)$ indicates an SPC electrode, and $\left(^{w}\right)$ indicates chambers with a $1 \mathrm{~mm}$ PMMA waterproofing.

\begin{tabular}{|c|c|c|c|c|c|c|c|}
\hline \multirow[b]{3}{*}{ chamber $\left[\mathrm{V}\left(\mathrm{cm}^{3}\right)\right]$} & \multicolumn{2}{|c|}{ 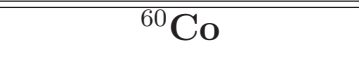 } & \multicolumn{5}{|c|}{$7 \mathrm{MV}$} \\
\hline & \multicolumn{2}{|c|}{$k_{Q}^{m a g} \equiv k_{B}(0.35 \mathbf{T})$} & \multirow[t]{2}{*}{$k_{Q}(\mathbf{0 T})$} & \multicolumn{2}{|c|}{$k_{Q}^{\operatorname{mag}}(1.5 \mathbf{T})$} & \multicolumn{2}{|c|}{$k_{B}(1.5 \mathbf{T})$} \\
\hline & $\|_{c h}$ & $\|_{p h}$ & & $\|_{c h}$ & $\|_{p h}$ & $\|_{c h}$ & $\|_{p h}$ \\
\hline & \multicolumn{7}{|c|}{ Exradin } \\
\hline A12 (0.65) & 1.0001 & 0.9999 & 0.9899 & 0.9882 & 0.9839 & 0.9983 & 0.9940 \\
\hline $\mathrm{A} 19(0.62)$ & 1.0006 & 0.9999 & 0.9875 & 0.9882 & 0.9839 & 1.0007 & 0.9964 \\
\hline $\mathrm{A} 2(0.54)$ & 1.0000 & 0.9987 & 0.9883 & 0.9872 & 0.9836 & 0.9989 & 0.9952 \\
\hline $\mathrm{T} 2(0.54)$ & 1.0002 & 0.9992 & 0.9816 & 0.9820 & 0.9816 & 1.0004 & 0.9999 \\
\hline $\mathrm{A} 12 \mathrm{~S}(0.25)$ & 0.9999 & 0.9983 & 0.9883 & 0.9867 & 0.9845 & 0.9984 & 0.9962 \\
\hline A18 (0.125) & 0.9991 & 1.0003 & 0.9885 & 0.9867 & 0.9856 & 0.9981 & 0.9971 \\
\hline $\mathrm{A} 1(0.057)$ & 0.9984 & 0.9992 & 0.9894 & 0.9857 & 0.9878 & 0.9962 & 0.9983 \\
\hline A1SL (0.057) & 0.9992 & 0.9999 & 0.9890 & 0.9857 & 0.9873 & 0.9966 & 0.9983 \\
\hline $\mathrm{A} 14^{*}(0.016)$ & 0.9933 & 0.9947 & 0.9951 & 0.9670 & 0.9779 & 0.9718 & 0.9827 \\
\hline $\mathrm{T} 14^{*}(0.016)$ & 0.9919 & 0.9948 & 0.9884 & 0.9584 & 0.9723 & 0.9696 & 0.9837 \\
\hline A14SL* (0.016) & 0.9932 & 0.9939 & 0.9932 & 0.9659 & 0.9756 & 0.9725 & 0.9823 \\
\hline \multirow[t]{2}{*}{$\mathrm{A} 16^{*}(0.016)$} & 0.9945 & 0.9950 & 0.9988 & 0.9588 & 0.9818 & 0.9600 & 0.9830 \\
\hline & \multicolumn{7}{|c|}{ PTW } \\
\hline PTW 30010 (0.6) & 0.9961 & 0.9982 & 0.9858 & 0.9731 & 0.9791 & 0.9872 & 0.9932 \\
\hline $\mathbf{P T W ~ 3 0 0 1 1}^{w}(0.6)$ & 0.9993 & 0.9999 & 0.9866 & 0.9787 & 0.9875 & 0.9920 & 1.0009 \\
\hline PTW 30012 ${ }^{w}(0.6)$ & 0.9952 & 0.9974 & 0.9878 & 0.9750 & 0.9817 & 0.9870 & 0.9938 \\
\hline PTW 30013 (0.6) & 0.9957 & 0.9983 & 0.9838 & 0.9720 & 0.9775 & 0.9881 & 0.9937 \\
\hline $31006(0.015)$ & 0.9989 & 0.9999 & 0.9869 & 0.9738 & 0.9822 & 0.9867 & 0.9953 \\
\hline $31010(0.125)$ & 0.9969 & 0.9986 & 0.9883 & 0.9817 & 0.9789 & 0.9933 & 0.9905 \\
\hline $31016(0.016)$ & 0.9979 & 0.9992 & 0.9850 & 0.9813 & 0.9842 & 0.9963 & 0.9992 \\
\hline \multirow[t]{2}{*}{$31014(0.015)$} & 0.9982 & 0.9988 & 0.9843 & 0.9795 & 0.9836 & 0.9951 & 0.9992 \\
\hline & \multicolumn{7}{|c|}{ IBA } \\
\hline FC65-G (0.65) & 0.9948 & 0.9970 & 0.9882 & 0.9799 & 0.9796 & 0.9917 & 0.9914 \\
\hline FC65-P (0.65) & 0.9949 & 0.9961 & 0.9881 & 0.9798 & 0.9782 & 0.9917 & 0.9901 \\
\hline FC23-C (0.23) & 1.0000 & 0.9999 & 0.9889 & 0.9869 & 0.9861 & 0.9980 & 0.9972 \\
\hline CC25 (0.25) & 0.9988 & 0.9988 & 0.9891 & 0.9878 & 0.9859 & 0.9987 & 0.9968 \\
\hline CC13 (0.13) & 1.0000 & 0.9997 & 0.9887 & 0.9877 & 0.9857 & 0.9990 & 0.9969 \\
\hline CC08 (0.08) & 0.9992 & 0.9989 & 0.9882 & 0.9858 & 0.9856 & 0.9975 & 0.9973 \\
\hline $\mathrm{CC} 04(0.04)$ & 0.9987 & 0.9990 & 0.9877 & 0.9849 & 0.9876 & 0.9971 & 0.9998 \\
\hline CC01 (0.01) & \multicolumn{6}{|c|}{ other } & 0.9889 \\
\hline $\mathrm{NE}^{2581^{w}}(0.6)$ & 0.9995 & 1.0000 & 0.9819 & 0.9812 & 0.9830 & 0.9993 & 1.0011 \\
\hline NE $2571^{w}(0.6)$ & 0.9948 & 0.9779 & 0.9890 & 0.9779 & 0.9813 & 0.9888 & 0.9922 \\
\hline $\mathrm{NE}^{2561}{ }^{w}(0.325)$ & 0.9956 & 0.9951 & 0.9907 & 0.9870 & 0.9783 & 0.9963 & 0.9875 \\
\hline $\mathrm{PR06C} / \mathrm{G}^{w}(0.65)$ & 1.0007 & 0.9995 & 0.9908 & 0.9894 & 0.9881 & 0.9986 & 0.9973 \\
\hline
\end{tabular}


Simulations with the $6 \mathrm{MV}$ Varian TrueBeam at $0.35 \mathrm{~T}$ for the recommended reference chambers in the TG-51 addendum (bold chambers in Table 7.1) produced $\mathrm{k}_{B}$ values, for $\|_{p h}$ and $\|_{c h}$, within $0.2 \%$ of those for ${ }^{60} \mathrm{Co}$ at $0.35 \mathrm{~T}$ (not shown). The only exceptions to this were the Exradin A1, having a $\mathrm{k}_{B}$ for $\|_{c h}$ of $1.0008 \pm 0.0007$ which is a $0.23 \%$ deviation from the ${ }^{60}$ Co results, and the NE 2571 , having a $\mathrm{k}_{B}$ for $\|_{p h}$ of $0.9972 \pm 0.0006$ which is a $0.27 \%$ change from the ${ }^{60}$ Co value. These calculations add to the results presented in Fig. 7.4, and demonstrate that the $\mathrm{k}_{B}$ values listed in Table 7.1 can be applied to higher beam qualities.

The PTW $30013 \mathrm{k}_{B}$ value at $1.5 \mathrm{~T}$ differs from Spindeldreier et al. by about $(0.4 \pm 0.14) \%$ in the $\|_{c h}$ orientation, and by less than $0.24 \%$ for the $90^{\circ}$ and $270^{\circ}$ orientations (when accounting for the adjusted sensitive air volume). Table 7.2 gives the O'Brien et al. ${ }^{26}$ calculated $\|_{c h}$ orientation $\mathrm{k}_{B}$ values and the percent difference from those calculated in this work. For the Exradin A19, PTW 30013, and PTW 30012 the differences are on the order of half a percent and within a few statistical uncertainties. However, the other chambers produce difference near or above $1 \%$. The differences are unexplained at present, but the close agreement between $\mathrm{k}_{Q}$ values calculated in this work and those in the TG-51 addendum provide more assurance in the present EGSnrc values.

Table 7.2: The $\|_{c h}$ orientation $\mathrm{k}_{B}$ values of O'Brien et al. and this work, and $\Delta \mathrm{k}_{B}$, the percent difference between those values. The NE 2571 is simulated with a water-proof sleeve.

\begin{tabular}{lccc}
\hline \hline & ${\text { O'Brien } \text { et } \text { al. }^{\mathbf{2 6}} \mathbf{k}_{B}}$ This work $\mathbf{k}_{B}$ & $\Delta \mathbf{k}_{B} \mathbf{( \% )}$ \\
\hline PTW 30013 & $0.994(2)$ & $0.9881(6)$ & $0.6 \pm 0.2$ \\
PTW 30012 & $0.992(3)$ & $0.9870(6)$ & $0.5 \pm 0.3$ \\
PTW 30011 & $1.000(3)$ & $0.9920(6)$ & $0.8 \pm 0.3$ \\
PTW 30010 & $0.996(3)$ & $0.9871(6)$ & $0.9 \pm 0.3$ \\
NE 2571 & $1.001(2)$ & $0.9888(7)$ & $1.2 \pm 0.2$ \\
Exradin A19 & $1.005(3)$ & $1.0007(8)$ & $0.4 \pm 0.3$ \\
\hline \hline
\end{tabular}




\subsection{Impact of the ICRU-90 recommendation on $\mathrm{k}_{Q}$}

The ICRU-90 ${ }^{80}$ report provides recommendations for ionizing radiation dosimetry. These update the recommendations made by the ICRU-3 $37^{79}$ report, and most relevant are the changes to the mean excitation energy (I) for graphite and water. The I-value differences between ICRU-37 and ICRU-90 are given in Table 7.3. In addition, for graphite, ICRU-90 uses the $2.265 \mathrm{~g} / \mathrm{cm}^{3}$ rather than the $1.70 \mathrm{~g} / \mathrm{cm}^{3}$ density correction.

Table 7.3: Comparison of the recommendations made by the ICRU-37 and ICRU90 reports for the values for the mean excitation energy. The ICRU-90 report also recommends the use of the $2.265 \mathrm{~g} / \mathrm{cm}^{3}$ density correction for graphite.

\begin{tabular}{lcc}
\hline \hline & ICRU-37 & ICRU-90 \\
\hline $\mathbf{I}_{\text {water }}(\mathbf{e V})$ & 75 & 78 \\
$\mathbf{I}_{\text {graphite }}(\mathbf{e V})$ & 78 & 81 \\
\hline \hline
\end{tabular}

The impact of these recommendations is determined by calculating $\mathrm{k}_{Q}$ for the 7 MV MRgRT photon spectrum, using the mean excitation energies and density corrections given by either ICRU-37 or ICRU-90 for the NE 2571, PTW 30011, PTW 30013, and Exradin A19 chambers. These chambers are selected because the first three have a graphite wall, the NE 2571 and PTW 30013 have aluminium electrodes, the PTW 30011 has a graphite electrode, and the Exradin A19 has a plastic wall and electrode. The percent difference, $\Delta k_{Q}^{I C R U}$, between the correction factors obtained for ICRU-37, $k_{Q-37}$, and ICRU-90, $k_{Q-90}$, are determined using:

$$
\Delta k_{Q}^{I C R U}=100 \% \times \frac{k_{Q-90}-k_{Q-37}}{k_{Q-37}} .
$$

\subsubsection{Results and Discussion}

Table 7.4 provides $k_{Q-37}, k_{Q-90}$, and $\Delta k_{Q}^{I C R U}$ values for the four chambers simulated. The TG-51 addendum $\mathrm{k}_{Q}$ values are given for comparison. All variations in $\mathrm{k}_{Q}$ are 
within $0.1 \%$ of unity. The changes are well below the expected change in $\mathrm{k}_{Q}$ of $-0.2 \%$ given by ICRU-90 ${ }^{80}$. A calculation of $\mathrm{k}_{B}$ for the NE 2571 showed a $(0.024 \pm 0.025) \%$ change in the value when using the different ICRU recommendations (i.e., no impact on $\mathrm{k}_{B}$ is observed). Deviations from the TG-51 addendum fit value are at most $0.05 \%$, for the NE 2571. A larger difference of $0.42 \%$ is seen between TG-51 values and $k_{Q-37}$ for the PTW 30013. This is attributed to noted changes in the ion chamber model.

Table 7.4: $\mathrm{k}_{Q}$ values calculated using the TG-51 addendum fits, ICRU-37, and ICRU90 recommendation and the associated percent difference (between the ICRU results) using the $7 \mathrm{MV}$ MRgRT photon spectrum. The statistical uncertainty on the TG-51 and simulated $\mathrm{k}_{Q}$ values is $\lesssim 0.07 \%$ and $0.02 \%$, respectively, and $0.03 \%$ on $\Delta k_{Q}^{I C R U}$.

\begin{tabular}{lcccc}
\hline \hline & $k_{Q-T G-51}$ & $k_{Q-37}$ & $k_{Q-90}$ & $\Delta k_{Q}^{I C R U}(\boldsymbol{\%})$ \\
\hline NE 2571 & 0.9883 & 0.98877 & 0.98886 & 0.01 \\
Exradin A19 & 0.9871 & 0.98739 & 0.98668 & -0.06 \\
PTW 30011 & 0.9871 & 0.98702 & 0.98712 & 0.01 \\
PTW 30013 & 0.9875 & 0.98330 & 0.98359 & 0.03 \\
\hline \hline
\end{tabular}

These results demonstrate that the change in $\mathrm{k}_{Q}$ due to the new recommendations are minimal for this beam quality. Although a more detailed study of the effects at different beam-qualities and other chambers is required, these results indicate that no major inaccuracies would be introduced by continuing to use the $\mathrm{k}_{B}$ values calculated here with the ICRU Report 37 stopping powers and the TG-51 addendum ${ }^{23}$ fits for $\mathrm{k}_{Q}$, which have a fit uncertainty of $\lesssim 0.1 \%$.

\section{5 $\mathbf{k}_{Q}^{\operatorname{mag}}$ and $\mathbf{k}_{B}$ for parallel-plate chambers}

Although the TG-51 addendum precludes the use of parallel-plate chambers for clinical reference dosimetry because of the instability of $\mathrm{N}_{D, w}^{60} \mathrm{Co} 23$, Muir and Rogers ${ }^{73}$ have provided $\mathrm{k}_{Q}$ correction factors for use with several parallel-plate chambers. To date, no explicit calculation of correction factors for parallel-plate chambers in magnetic fields has been published. 
The NACP-02, Roos, and Markus parallel-plate chambers ${ }^{73}$ are simulated for the same conditions as in Sec. 7.3. Here, the $\|_{c h}$ geometry is with the magnetic field perpendicular to the incoming photon beam (i.e., the magnetic field is directed along the $\mathrm{x}$-axis, the photon beam is incoming along the z-axis, and the front window of the parallel-plate chamber faces towards the photon beam). Due to the radial symmetry of the parallel-plate chamber, there is no inherent angular dependence in $\mathrm{k}_{Q}^{\text {mag }}$ and $\mathrm{k}_{B}$, unlike with the cylindrical ion chambers.

\subsubsection{Results and Discussion}

The TG-51 protocol and the addendum do not recommend the use of parallel-plate chambers for photon-beam reference dosimetry, however, the well defined point of measurement of these chambers makes them useful for beam-quality measurements (i.e., depth-dose curves). In the context of magnetic fields, these chamber are symmetric about their central axis, which eliminates any effects due to rotations about that axis. Due to this symmetry, $\|_{c h}$ simply indicates the configuration in which the magnetic field is perpendicular to the incoming photon beam, and the $\|_{p h}$ notation is as in the previous section.

In Table $7.5, \mathrm{k}_{Q}, \mathrm{k}_{B}$, and $\mathrm{k}_{Q}^{m a g}$ are provided for the Markus, Roos, and NACP-02 parallel-plate chambers for ${ }^{60} \mathrm{Co}$ and $7 \mathrm{MV}$. Agreement between these $\mathrm{k}_{Q}$ values and those obtained using the functional fits provided by Muir et al. ${ }^{75}$ is within $0.12 \%$. The effect of the magnetic field is clearly much larger for these chamber, for both photon energies. The $\|_{p h} \mathrm{k}_{B}$ values are consistently a smaller correction than the $\|_{c h}$ values, which are as large as an $8.9 \%$ correction for the case of the Roos chamber. 
Table 7.5: As in Table 7.1 but for parallel-plate chambers.

\begin{tabular}{|c|c|c|c|c|c|c|c|}
\hline & \multicolumn{2}{|c|}{${ }^{60} \mathrm{Co}$} & \multicolumn{5}{|c|}{$7 \mathrm{MV}$} \\
\hline & \multicolumn{2}{|c|}{$k_{Q}^{m a g} \& k_{B}(0.35 \mathrm{~T})$} & \multirow[t]{2}{*}{$k_{Q}(\mathbf{0 T})$} & \multicolumn{2}{|c|}{$k_{Q}^{\operatorname{mag}}(1.5 \mathbf{T})$} & \multicolumn{2}{|c|}{$k_{B}(1.5 \mathrm{~T})$} \\
\hline & $\|_{c h}$ & $\|_{p h}$ & & $\|_{c h}$ & $\|_{p h}$ & $\|_{c h}$ & $\|_{p h}$ \\
\hline Markus & 0.9948 & 0.9994 & 0.9920 & 1.0185 & 0.9796 & 1.0267 & 0.9875 \\
\hline Roos & 0.9576 & 0.9970 & 0.9889 & 0.9012 & 0.9739 & 0.9113 & 0.9849 \\
\hline NACP-02 & 0.9644 & 0.9971 & 0.9870 & 0.9322 & 0.9681 & 0.9445 & 0.9809 \\
\hline
\end{tabular}

A slight three degree tilt of the chambers was simulated with the $7 \mathrm{MV}$ beam at 1.5 T. In the $\|_{p h}$ orientation, there was no change in the calculated cavity dose-to-air, but in the $\|_{c h}$ orientation the dose varied by as much as $(0.28 \pm 0.09) \%,(0.44 \pm 0.05) \%$, and $(0.39 \pm 0.07) \%$ for the Markus, Roos, and NACP-02 chambers, respectively. These variations are similar to the variations seen for the cylindrical chambers, but these are produced with much smaller angular changes and demonstrate further uncertainty in using parallel-plate chambers for magnetic field reference dosimetry.

\subsection{Beam-quality specification}

As shown by O'Brien et al. ${ }^{26}$, for the MRgRT $7 \mathrm{MV}$ photon beam, changes in the location and magnitude of the maximum dose in a depth-dose curve in a water phantom produces changes in the value of $\% \mathrm{dd}(10)_{x}$. Here the DOSXYZnrc application is used to obtain $\% \operatorname{dd}(10)_{x}$ and $\mathrm{TPR}_{10}^{20}$ as a function of magnetic field for all of the photon beams listed in Table 7.6, with the exception of the Varian TrueBeam spectrum.

The $\% \operatorname{dd}(10)_{x}$ beam-quality specifier is determined by calculating percent-depthdose curves in a $30 \times 30 \times 30 \mathrm{~cm}^{3}$ water phantom. The photon beams, incoming along the $\mathrm{z}$-axis, have a surface field size of $10 \times 10 \mathrm{~cm}^{2}$, at the water phantom surface, and an SSD of $100 \mathrm{~cm} .10 \mathrm{~cm}$ of air is simulated on either end of the phantom along the direction of the beam to ensure that any electrons being curved by the magnetic field can return to the water surface. Dose is scored in voxels along the central axis of the 
beam in a $1 \mathrm{~cm}$ wide region along the $\mathrm{x}$ and $\mathrm{y}$ axis and $0.3 \mathrm{~cm}$ thick along the $\mathrm{z}$-axis for the first and last $5 \mathrm{~cm}$ of the water phantom and $1 \mathrm{~cm}$ thick along the $\mathrm{z}$-axis for the central region. ${ }^{60}$ Co simulations are performed with $0.1 \mathrm{~cm}$ thick regions along the z-axis.

Table 7.6: Photon beam sources and their respective beam-quality specifiers, $\% \mathrm{dd}(10)_{x}$, photon component of the percent-depth-dose at $10 \mathrm{~cm}$, and $\mathrm{TPR}_{10}^{20}$, ratio of tissue phantom ratios at 20 and $10 \mathrm{~cm}$ for a $10 \times 10 \mathrm{~cm}^{2}$ field size at the point of measurement and an SDD of $100 \mathrm{~cm}$. The statistical uncertainty on the beam-quality specifiers is $0.15 \%$.

\begin{tabular}{|c|c|c|c|}
\hline beam & nominal energy & $\overline{\% \operatorname{dd}(10)_{x}}$ & $\overline{T P R_{10}^{20}}$ \\
\hline Co60 ${ }^{74}$ & - & 58.6 & 0.568 \\
\hline \multirow[t]{2}{*}{ Elekta SL $25^{53}$} & $6 \mathrm{MV}$ & 67.2 & 0.672 \\
\hline & $25 \mathrm{MV}$ & 82.6 & 0.794 \\
\hline \multirow[t]{2}{*}{ Varian Clinical $^{53}$} & $6 \mathrm{MV}$ & 66.4 & 0.663 \\
\hline & $10 \mathrm{MV}$ & 73.8 & 0.733 \\
\hline Varian TrueBeam ${ }^{81}$ & $6 \mathrm{MV}$ (FFF) & 63.3 & 0.625 \\
\hline $\operatorname{MRgRT}^{65}$ & $7 \mathrm{MV}$ & 69.4 & 0.695 \\
\hline
\end{tabular}

The $\mathrm{TPR}_{10}^{20}$ is determined by obtaining the dose centered at $10 \mathrm{~cm}$ and $20 \mathrm{~cm}$ depth in a $1 \mathrm{~cm}^{3}$ voxel in the water phantom described above and with a parallel photon beam incoming along the $\mathrm{z}$-axis with a field size of $10 \times 10 \mathrm{~cm}^{2}$. This method did not produce any statistically different $\mathrm{TPR}_{10}^{20}$ values when compared to the regular method used for calculating TPR ${ }_{10}^{20}$. The only exception to this was the higher-energy Elekta SL25 $25 \mathrm{MV}$ photon beam. For this photon energy two individual simulations were performed using the traditional method for determining $\mathrm{TPR}_{10}^{20}\left(2 \mathrm{SSDs}, 10 \times 10 \mathrm{~cm}^{2}\right.$ at either $10 \mathrm{~cm}$ or $20 \mathrm{~cm}$ depth, and a photon point source - as in Figure 1.3).

Comparing the simulated $\mathrm{TPR}_{10}^{20}$ values in Table 7.6 to those calculated using the formula from Kalach and Rogers ${ }^{24}$ shows less than $0.2 \%$ difference between the two results, with the exception of the Varian TrueBeam 6 MV spectrum. This demonstrates that these beams, again except for the TrueBeam, act as clinical filtered beams.

In addition to the $\% \mathrm{dd}(10)_{x}$ and $\mathrm{TPR}_{10}^{20}$ values as a function of magnetic field, 
the percent-depth-dose curves for the ${ }^{60} \mathrm{Co}$, MRgRT $7 \mathrm{MV}$, and Elekta SL25 $25 \mathrm{MV}$ photons beams are calculated for 0,1 , and $2 \mathrm{~T}$ magnetic fields.

\subsubsection{Results and Discussion}

Due to the magnetic field's influence on electron trajectories there can be substantial changes to entry and exit doses near interfaces of materials with very different densities. O'Brien et al. ${ }^{26}$ demonstrated that this effect leads to changes in the location and magnitude of the maximum dose in the percent-depth-dose curves (PDDs), and this causes a change in the $\% \mathrm{dd}(10)_{x}$ beam-quality specifier. Since $\mathrm{TPR}_{10}^{20}$ is based on the dose at depth in the water phantom, it is expected that this beam-quality specifier will not change much due to the presence of the magnetic field.

In Fig. 7.5, the central axis percent PDDs can be seen for ${ }^{60} \mathrm{Co}, 7 \mathrm{MV}$, and $25 \mathrm{MV}$ with a magnetic field perpendicular to the incoming photon beam. The effect of the magnetic field can be seen near the entry and exit surfaces of the water phantom, and are most evident at the higher energies for which the secondary electrons have a larger range of travel and are able to deposit their kinetic energy further along the magnetic field induced trajectory. Interestingly, a secondary "build-up" region is observed at the end of the phantom for the $25 \mathrm{MV}$ beam with a $2 \mathrm{~T}$ field, due to electrons curving back to the water surface. Setting the magnetic field to be parallel with the incoming photon beam did not produce notable changes in the PDDs. 

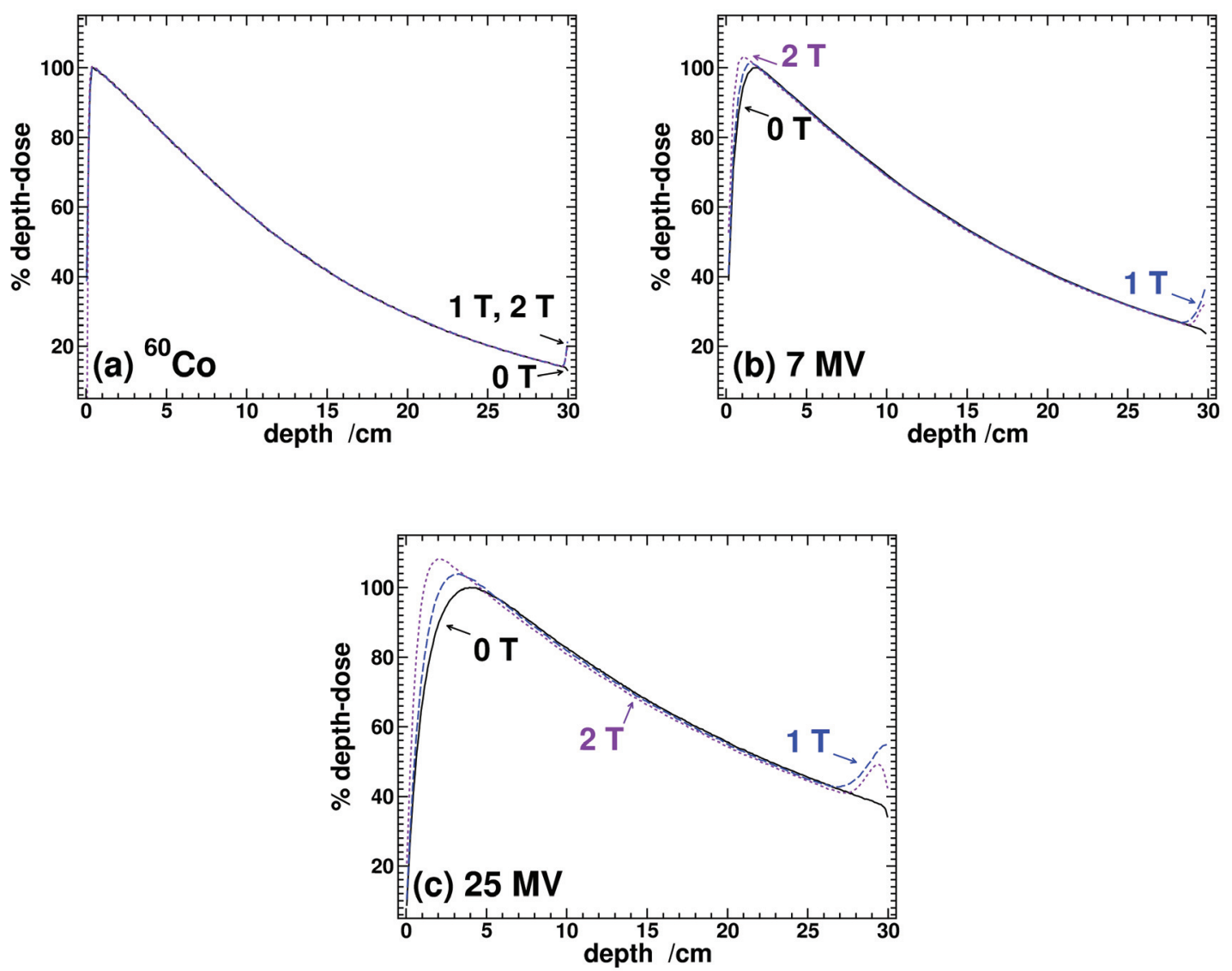

Figure 7.5: Central-axis percent-depth-dose curves for (a) ${ }^{60} \mathrm{Co}$ (b) MRgRT 7 MV and (c) Elekta SL25 $25 \mathrm{MV}$ photon beams with either no magnetic field or a 1 or $2 \mathrm{~T}$ magnetic fields perpendicular to the incoming photon beam. Each curve is normalized to the maximum value of the $0 \mathrm{~T}$ simulation. The beams include no incident electron contamination.

To evaluate the variation in $\% \operatorname{dd}(10)_{x}$, the beam-quality specifier is plotted as a function of magnetic field for a variety of beam energies in Fig. 7.6. A clear dependence on the magnetic field is seen, and the effect becomes larger with increasing photon beam energy. Between the $0 \mathrm{~T}$ and $1.5 \mathrm{~T}$, the ${ }^{60} \mathrm{Co}$ results show no statistically significant differences in $\% \mathrm{dd}(10)_{x}$, while for the $7 \mathrm{MV}$ and $25 \mathrm{MV}$ photon beams there is an absolute decrease in $\% \mathrm{dd}(10)_{x}$ of $(1.89 \pm 0.10) \%$ and $(6.20 \pm 0.10) \%$, respectively, due to primarily the increase in $\mathrm{D}_{\max }$. O'Brien et al. ${ }^{26}$ observed a comparable $1.7 \%$ decrease in $\% \mathrm{dd}(10)_{x}$ for the $7 \mathrm{MV}$ MRgRT photon beam. Although it would be possible to 
produce a functional correction that would allow the user to correct their measured $\% \operatorname{dd}(10)_{x}$ value for a given magnetic field and photon energy, the non-linear nature of the effect (especially at high energies) and the beam quality dependence makes this less feasible.

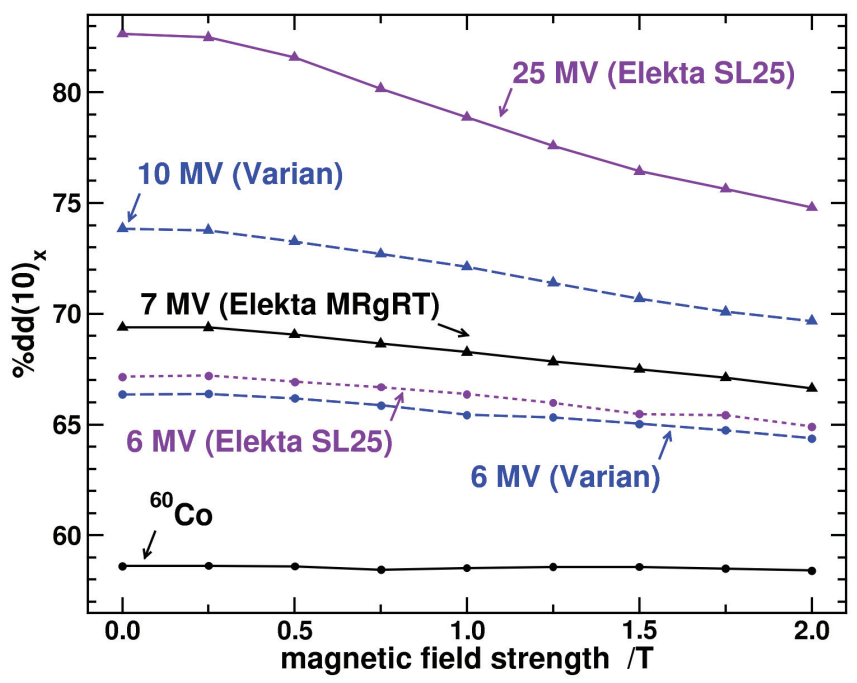

Figure 7.6: The value of the photon component of the percent-depth-dose curve at $10 \mathrm{~cm}$ in water $\left(\% \mathrm{dd}(10)_{x}\right)$ as a function of magnetic field for the indicated beams.

In Fig. 7.7 the $\mathrm{TPR}_{10}^{20}$ specifier is plotted as a function of magnetic field. For all photon energies, variations in $\mathrm{TPR}_{10}^{20}$ are within $0.36 \%$ across all magnetic fields. The $7 \mathrm{MV}$ spectrum $\mathrm{TPR}_{10}^{20}$ values for $0 \mathrm{~T}$ and $1.5 \mathrm{~T}$ match those calculated by O'Brien et $a l .{ }^{26}$ who found a roughly $0.2 \%$ difference in $\mathrm{TPR}_{10}^{20}$ between these two magnetic fields. This makes $\mathrm{TPR}_{10}^{20}$ the preferred beam-quality specifier in magnetic fields and a functional relationship between $\mathrm{TPR}_{10}^{20}$ and $\% \mathrm{dd}(10)_{x}$, such as that provided by Kalach and Rogers ${ }^{24}$, should be used to convert to $\% \mathrm{dd}(10)_{x}$ for use with the TG-51 protocol. 


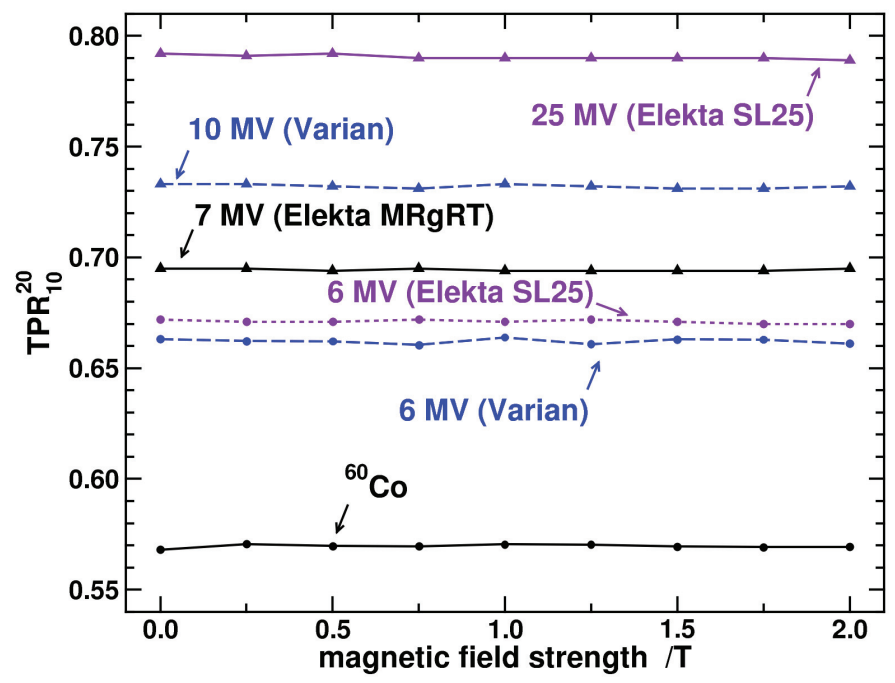

Figure 7.7: Tissue phantom ratio at 20 and $10 \mathrm{~cm}$ in water as a function of magnetic field strength for the indicated beams.

\section{7 $\quad$ Summary}

The presence of the magnetic field presents some challenges to performing ion chamber dosimetry in magnetic fields, but these are not insurmountable. As shown in Eqs. 1.8 and 1.9, there are two methods of accounting for the effects of the magnetic field in the framework of the TG-51 protocol. Due to the reduced beam quality dependence in the values of $\mathrm{k}_{B}$, the magnetic field correction factor, it may be optimal to use this factor along with the conventional $\mathrm{k}_{Q}$ factor to account for beam quality variation. In this work, both $\mathrm{k}_{B}$ and $\mathrm{k}_{Q}^{m a g}$ have been determined for relevant orientations, beam energies, and magnetic fields, for 32 cylindrical ionization chambers. The effect of air gaps is not considered since it is assumed that measurements are performed in a water phantom.

The magnetic field correction factor $\mathrm{k}_{B}$ was found to be within $1 \%$ of unity for most chambers and for both ${ }^{60} \mathrm{Co}$ at $0.35 \mathrm{~T}$ and $7 \mathrm{MV}$ at $1.5 \mathrm{~T}$. Chambers with a larger correction were found to be those that have a high-Z (steel, aluminium, steel-coppersilver) electrode. As in the TG-51 addendum ${ }^{23}$, the SPC electrode chambers are not 
recommended for dosimetry in magnetic fields, and overall the recommendations of the TG-51 addendum should be followed for chamber selection. The existing magnetic field transport code has been shown to agree well with experimental measurements of the ion chamber response as a function of magnetic field when the sensitive volume is adjusted for potential "dead" volume regions. To provide full validation of the correction factors produced in this work and others, experimental measurements are necessary for the orientation in which the magnetic field is parallel to the long axis of the chamber and perpendicular to the incoming photon beam.

The ICRU-90 recommended mean excitation energies and density corrections produce changes in $\mathrm{k}_{Q}$, for the $7 \mathrm{MV}$ MRgRT photon spectrum, within $0.1 \%$ of the value obtained using the recommendations in ICRU-37. No impact of using the new ICRU-90 values on $\mathrm{k}_{B}$ is observed.

The use of parallel-plate chambers for magnetic field dosimetry is also explored, and results show that due to the lack of an orientation which reduces the effect of the magnetic field, as for cylindrical chambers, these chambers suffer from larger $\mathrm{k}_{B}$ correction factors in both the $\|_{p h}$ and $\|_{c h}$ orientations. Further, even a small $3^{\circ}$ tilt in the orientation of the parallel-plate chambers produced up to a $0.44 \%$ change in the $\mathrm{k}_{Q}^{m a g}$. This variation can be compared to the cylindrical chambers, which experience a similar change of $\mathrm{k}_{Q}^{m a g}$ but for a $10^{\circ}$ misalignment. Overall, these results indicate that parallel-plate chambers are not well suited for use in magnetic field reference dosimetry.

The effect of the magnetic field on the $\% \operatorname{dd}(10)_{x}$ beam-quality specifier is determined to be on the order of several percent for relevant beam energies. The variation in $\% \operatorname{dd}(10)_{x}$ is non-linear and is magnetic field and beam quality dependent. Due to the stability of $\mathrm{TPR}_{10}^{20}$ across all studied beam energies and magnetic fields, this beamquality specifier should be used and appropriate equations applied to convert $\mathrm{TPR}_{10}^{20}$ to $\% \operatorname{dd}(10)_{x}$ as required. 


\section{Chapter 8}

\section{Final overview and outlook}

\subsection{Summary and conclusions}

The aim of this work was to implement an efficient and reliable magnetic field transport code in EGSnrc with the purpose of evaluating the impact of the magnetic field on dosimetry protocols and dose distributions. The previous version of the magnetic field transport code that was available in EGS4, was officially made available in the January 2017 release of the EGSnrc code. This implementation is based on a one-point numerical integration technique of the magnetic field force, and consequently has a large impact on simulation efficiency. In chapter 2, the implementation produced in this work is described. Accuracy improvements such as a three-point numerical integration technique for the condensed history transport and the use of the analytical solution for charged particle transport in magnetic fields in vacuum for the single scatter mode are introduced. Further, a boundary crossing algorithm which works with arbitrary geometries and ensures that particles do not improperly cross into different regions is implemented. To maintain the accuracy of the numerical integration techniques, proper step-size restrictions and step-size scaling with magnetic field are introduced. By combining the three-point and one-point integration techniques for longer and shorter steps, respectively, the efficiency of the algorithm is improved.

In chapter 3, the code is validated by applying a magnetic field compatible Fano 
cavity test and by comparing to experimental measurements of the normalized ion chamber response as a function of magnetic field. The "full algorithm" that is used for all further simulations in this thesis is shown to pass the Fano cavity test in an ion chamber and slab geometry at the $0.1 \%$ level. This is compared to using only the one-point integration method which quickly diverges from the expected Fano cavity test value with increasing step sizes. Simulations of the NE 2571 ion chamber relative response as a function of magnetic field produced very good agreement, up to about $1 \mathrm{~T}$, with experimental results. Experimental unknowns such as air gaps around the chamber and the alignment of the incoming radiation beam was shown to produce variations which would account for the differences at the higher magnetic fields strengths. The introduction of the magnetic field code was also determined to increase computational time by $38 \%$ to $80 \%$ (depending on geometry). This increase in time is compared to more than doubling of the computation time if using only the one-point integration technique for the condensed history transport and the analytic solution for the single scatter (further increase in time would result from using one-point integration for both transport techniques).

The validated magnetic field transport algorithm is then applied, in chapter 4, to evaluate the importance of including heterogeneities in lung structure when performing treatment planning for MRgRT systems. Although a fairly simple geometry is simulated in this work, it is evident that air structures larger than $1 \mathrm{~mm}$ in diameter may lead to variations in dose distributions that would be missed by a homogeneous lung phantom. Simulations with a more realistic "tube" like structure show a slight reduction in the dose differences between the homogeneous and heterogeneous phantoms, and may indicate that the distribution of airways in the lung would average out some of the effects observed in this study. However any repeating structure or larger diameter regions may still have to be simulated.

In chapter 5 an extremely important effect on calculated ion chamber relative 
response in magnetic field is explored. The charge collecting electric field inside the ion chamber can fringe near the guard electrode. This fringing would not allow the main electrode to collect charges released into that volume and strictly this region should be omitted from the dose calculation in the MC models of the cylindrical ion chamber. The simulations presented in this chapter show that, by varying the size of the sensitive volume, the ion chamber dose in the presence of a magnetic field perpendicular to both the photon beam and the long axis of the chamber could change by several percent. These changes in sensitive volume produced results that improved agreement with experimental measurements. It is important to note that it is unreliable to treat the sensitive volume as a free parameter that could be adjusted until agreement with experiment is achieved. To address this effect, either the full electric field inside each of the chambers has to be modelled or an orientation in the magnetic field should be found where this effect is negligible.

By evaluating $\mathrm{k}_{Q}^{\text {mag }}$ as a function of angle with respect to the magnetic field in chapter 6 , the orientation in which the magnetic field is parallel to the long axis of the ion chamber and perpendicular to the photon beam is determined to be optimal for magnetic field clinical reference dosimetry. In this orientation the influence of the magnetic field on the dose to chamber air volume is significantly reduced and more importantly the impact of the size of the chamber sensitive volume is rendered negligible. This latter point is mainly because the Lorentz force does not sweep the electrons towards the tip or stem of the chamber, and the dose distribution in the chamber air volume is fairly uniform.

Chapter 7 deals with the calculation of $\mathrm{k}_{B}$ and $\mathrm{k}_{Q}^{\text {mag }}$ values for 32 cylindrical ion chamber in magnetic fields which are perpendicular or parallel to the photon beam. The optimal orientation that was determined in chapter 6 is used. A study of the beam quality dependence of $\mathrm{k}_{B}$ and $\mathrm{k}_{Q}^{\text {mag }}$ demonstrates, as expected, that $\mathrm{k}_{B}$ carries a much lower beam quality dependence than $\mathrm{k}_{Q}^{\text {mag }}$. This suggests that the $\mathrm{k}_{B}$ correction 
factor would be the preferred choice for accounting for the presence of the magnetic field in clinical reference dosimetry. A single $\mathrm{k}_{B}$ factor for each magnetic field could be determined for each chamber and applied at various beam qualities. The main advantage would be that if the user's machine varies slightly in beam quality there will not be a dramatic impact on the $\mathrm{k}_{B}$ that should used. The correction factors calculated for the cylindrical ion chamber in this chapter apply to a ${ }^{60} \mathrm{Co}$ beam in the presence of a $0.35 \mathrm{~T}$ magnetic field and a $7 \mathrm{MV}$ photon beam with a $1.5 \mathrm{~T}$ magnetic field. Magnetic field corrections larger than $1 \%$ are associated with ion chambers with higher density electrodes and the majority of chambers have $\mathrm{k}_{B}$ values within $1 \%$ of unity. Parallel-plate ionization chambers suffer from correction factors on the order of several percent and for certain cases nearly $10 \%$. The fact that there is no orientation that would reduce the impact of the magnetic field and the sensitivity of these chambers to slight changes in orientation, it is recommended that parallel-plate chambers should not be used for clinical reference dosimetry and their application may be limited even in relative dosimetry. Additionally, applying the new ICRU-90 recommendations for material physical properties for several chambers showed that there is little impact on MC calculated $\mathrm{k}_{Q}$ values.

An important component of clinical reference dosimetry is determining the beam quality of the linear accelerator. The $\% \mathrm{dd}(10)_{x}$ is the beam-quality specifier recommended in the TG-51 protocol, and TPR 10 is used in TRS-398. In chapter $7, \% \mathrm{dd}(10)_{x}$ is shown to change by several percent when a magnetic field is introduced, with a difference of $6.2 \%$ between the $0 \mathrm{~T}$ and $1.5 \mathrm{~T} \% \mathrm{dd}(10)_{x}$ value for a $25 \mathrm{MV}$ beam. The $\mathrm{TPR}_{10}^{20}$ beam-quality specifier does not show any statistically significant variation as a function of magnetic field, and is therefore the optimal beam-quality specifier for magnetic field clinical reference dosimetry.

Overall, an efficient and stable code for charged particle transport in magnetic fields has been introduced. This code has been benchmarked using the Fano test and 
comparison to experimental data. The code has been applied to clinically relevant calculations and to provide correction factors for ion chamber reference dosimetry in magnetic fields.

\subsection{Future work}

MRgRT is an evolving technology which will require a great deal of further research into the impact of magnetic fields on several aspects of treatment planning and dosimetry. To date, many effects, such as air gaps around ion chambers, changes in beam-quality specifiers, and the importance of correctly simulating the sensitive volume of ion chambers, have been determined. There are several scientific avenues in which the implemented code can be applied in this field.

An important point is to continue to verify the MC calculations being performed with the code developed in this work and by other groups. As highlighted in the chapters above, many of the measurements that have been performed are in orientations which make it difficult to fully validate MC magnetic field transport algorithms. Experimental work in the optimal orientation described in this work will be a crucial component in allowing for full benchmarking of the transport codes and verification of calculated magnetic field correction factors.

The emphasis of this thesis is on ion chamber calculations at specific reference depths. Often cylindrical and parallel-plate chambers are used for measuring depthdose curves or surface doses. The implemented magnetic field transport code should be applied to determine the reliability of using these chamber for relative dosimetry.

Treatment planning systems for MRgRT systems are used to produce inverse planning of patient doses. These systems include algorithms which account for the presence of the magnetic field and it will be extremely important to verify the results produced 
by these systems. This may include reproducing the inverse planning that these systems perform or using beam orientations produced by the planning systems to verify the dose distributions.

The calculations in this work deal with constant magnetic fields, and this is valid for magnetic resonance systems which require very uniform magnetic fields. However, extending the code to allow for particle motion in spatially varying magnetic fields would permit performing dosimetry simulations for the purposes of evaluating the impact of any variations of the MRI magnetic field.

Further, a study comparing the different distribution of magnetic field capable MC codes (such as Geant4, PENELOPE, and other implementations in EGSnrc) should be performed to evaluate any discrepancies. This would be a very important contribution in verifying many of the $\mathrm{MC}$ results that have been published.

Collectively these are a few of the research goals that will be required in creating clinical protocols for magnetic field clinical reference dosimetry and safe treatments with MRgRT systems. 


\section{Appendix A}

\section{Normalization of final direction vector}

Once $\Delta \vec{u}_{B}$ is determined it must be added to the direction of motion at the end of the $\mathrm{CH}$ step and the unit vector properties of the direction of motion must be maintained. One must be careful with the re-normalization to recognize that the direction component parallel to the magnetic field dose not change. Although this was recognized originally by Bielajew ${ }^{40}$, it does not appear to have been taken into account in the implementations in PENELOPE ${ }^{35}$ or by Tang and Bednarz ${ }^{39}$ where the applied normalization is given by

$$
\hat{u}_{f}=\frac{\hat{u}_{C H}+\Delta \vec{u}_{B}}{\left|\hat{u}_{C H}+\Delta \vec{u}_{B}\right|}
$$

where, $\hat{u}_{f}$ is the final direction of motion and $\hat{u}_{C H}$ is the direction of motion at the end of the $\mathrm{CH}$ step. In this approach components of the direction vector which should not be influenced by the magnetic field are scaled down in magnitude. The resulting effect is observed in Fig. A.1a, where motion in vacuum of a $0.5 \mathrm{MeV}$ electron initially directed at a $45^{\circ}$ angle with respect to the $\mathrm{z}$ axis is tracked in a $0.2 \mathrm{~T}$ magnetic field oriented in the z-direction. Deviations from the analytical solution are observed for $\delta_{u}$ values as low as 0.02 , and a noticeable change in the amplitude of oscillation due to the normalization problem is seen for $\delta_{u}=0.1$. Calculations with $\delta_{u}=0.2$ are not shown in panel (a) as the z-component of the direction became insignificant and particles travelled in perpetual circular orbits, i.e. trajectories did not reach past $10 \mathrm{~cm}$ depth along the z-axis. 


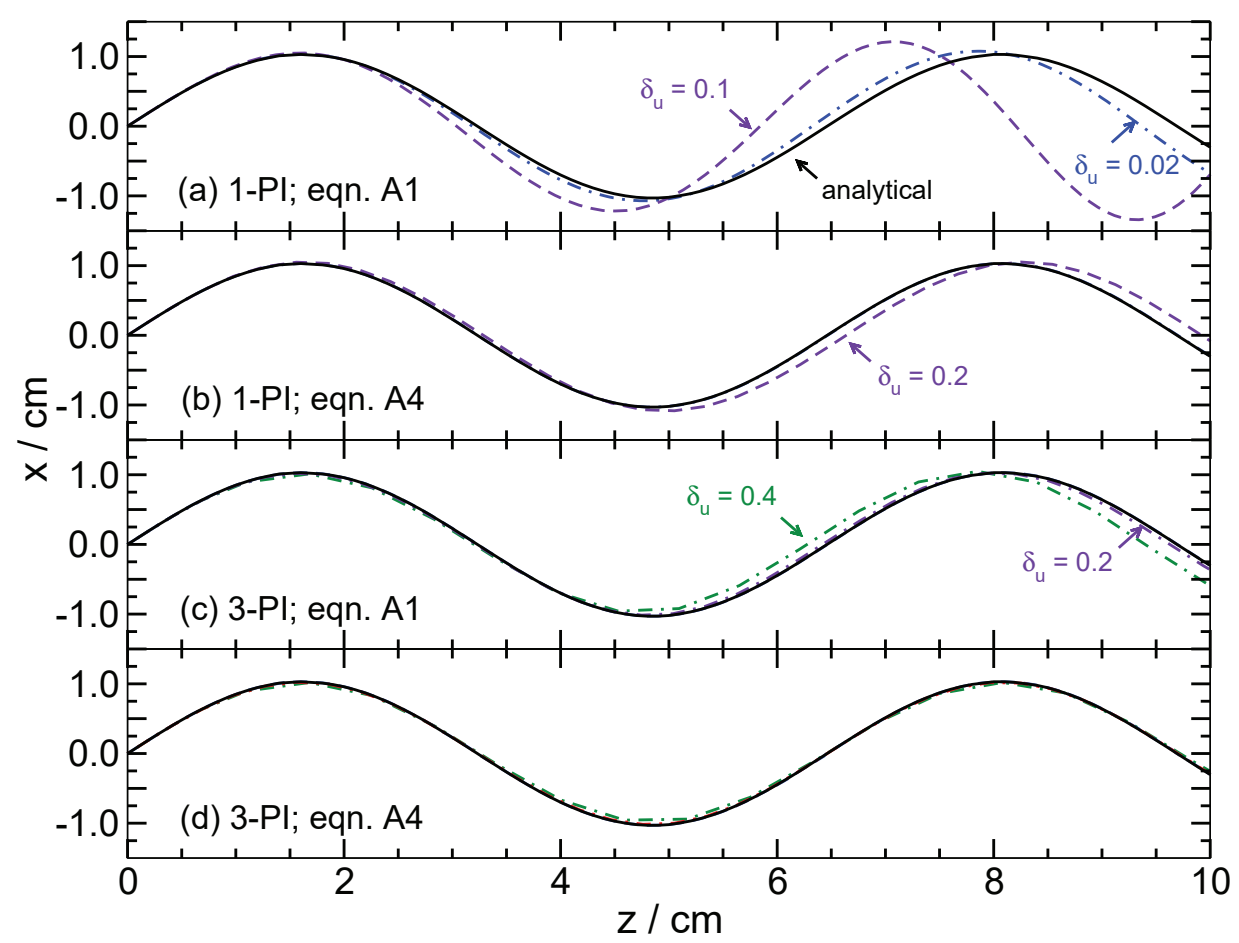

Figure A.1: $\mathrm{x}$-axis as a function of z-axis position for a $0.5 \mathrm{MeV}$ electron in a $0.2 \mathrm{~T}$ magnetic in z-direction in a near vacuum. The black line is the analytical solution. (a) 1-PI $\left(\delta_{u}\right.$ or 0.1 and 0.02$)$ normalized with Eq. A1, (b) as (a) but with Eq. A4, (c) 3-PI $\left(\delta_{u}\right.$ or 0.4 and 0.2$)$ normalized with Eq. A1, (d) as (c) but with Eq. A4. Numerical simulation results overlapping with the analytical solution are not labeled.

To conserve the momentum in the direction parallel to the magnetic field, the components of the direction vector parallel and perpendicular to the magnetic field are defined as:

$$
\vec{u}_{\|}=\hat{u}_{C H} \cdot \hat{B}
$$

and,

$$
\vec{u}_{\perp}=\hat{u}-\vec{u}_{\|}
$$

where $\hat{B}, \vec{u}_{\|}$, and $\vec{u}_{\perp}$ are the unit direction of the magnetic field, and the parallel and perpendicular components of the direction of motion, respectively. The effect of the magnetic field is obtained by using the individual magnitudes of these two components as in Eq. A4,

$$
\hat{u}_{f}=\vec{u}_{\|}+\frac{\vec{u}_{\perp}+\Delta \vec{u}_{B}}{\left|\vec{u}_{\perp}+\Delta \vec{u}_{B}\right|}\left|\vec{u}_{\perp}\right| .
$$


This normalization is applied in Fig. A.1b for the same energy and configuration as in panel (a). Here the $\delta_{u}=0.02 \mathrm{CH}$ solution follows the analytical solution perfectly. Slight deviations are observed for $\delta_{u}=0.2$. Using 3-PI, the $\delta_{u}=0.2$ solution also maintains adherence to the analytical propagation (Fig. A.1d). Further, using 3-PI appears to overcome the issues presented by using Eq. A1 (Fig. A.1c), but the appropriate normalization technique is applied for increased stability in the solution.

The component normalization technique provides improved tracking of particles in vacuum, and in low density media. Coupled to the higher order integration technique a larger value for the $\delta_{u}$ parameter is permissible, which corresponds to larger step-sizes and shorter computational times. 


\section{Appendix B}

\section{Condensed history $t_{\text {perp }}$ restriction}

Since boundary crossing is mediated by SS, the $\mathrm{CH}$ mode cannot cause regional transitions. Without the magnetic field code, the $\mathrm{CH}$ step is restricted to a maximum value of $t_{\text {perp }}$. If a magnetic field is present and Eq. 2.6 is used to adjust the position at the end of the $\mathrm{CH}$ step, the particle can step into an incorrect region even if the $\mathrm{CH}$ total step length was just $t_{\text {perp }}$ (Fig. B.1a). To maintain proper boundary transitions, it is important that when a magnetic field is present the displacement caused by the magnetic field, $\Delta \vec{x}_{B}$, is added to the step length taken in the $\mathrm{CH}$ calculation, $t_{C H}$, and this must be bounded to $t_{\text {perp }}$, as in Fig. B.1b.

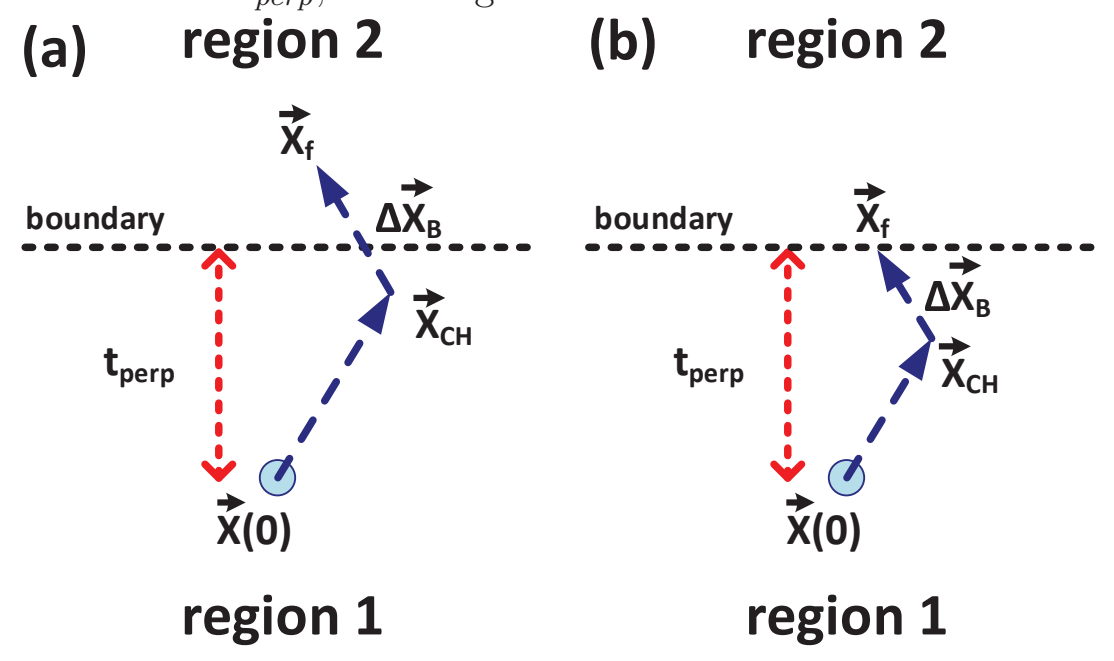

Figure B.1: A condensed history step equal to $t_{\text {perp }}$ in a magnetic field (a). The influence of the magnetic field causes the particle to fall into region two. Restricting the step-size using Eq. B3 ensures the final position is in region one (b). 
This is encoded as:

$$
t_{\text {perp }}=t_{C H}+\left|\Delta \vec{x}_{B}\right|
$$

Using Eq. 2.6 and $s=t_{C H}$, Eq. B1 can be rewritten as

$$
t_{p e r p}=t_{C H}+\frac{t_{C H} \eta}{2}\left|\Delta \vec{u}_{B}\right|
$$

Here, the value of $\eta$ is estimated by using the ESTEPE variable, which controls the maximal fractional energy loss along a step in EGSnrc. Eq. 2.3 is used to evaluate $\Delta u_{B}$ and substitution into Eq. B2 gives:

$$
t_{\text {perp }}=t_{C H}+t_{C H}^{2}\left|\frac{q(\eta c)^{2}}{4 E v_{i}} \vec{v}(0) \times \vec{B}\right| .
$$

This is a quadratic equation which can be solved for $t_{C H}$ values that satisfy the expression. The maximum value is used as the upper bound for the $\mathrm{CH}$ mode restriction. By using this algorithm proper boundary transitions are maintained and no errors in scoring are encountered. Since the solution to the quadratic equation does not include any variation in velocity or direction of motion during the step, it is possible that the 3-PI results in a total step larger than $t_{C H}$. To avoid this, the magnitude of the change in position induced by the magnetic field is calculated and compared to the current $t_{\text {perp }}$ value, and the 1-PI calculation is used if $t_{p e r p}$ is found to be smaller. This type of event occurs fairly rarely and does not influence the overall dose calculation results. 


\section{Appendix C}

\section{Energy loss over elastic mean free path}

One of the assumptions of the magnetic field transport algorithm is that in the single scattering mode there is little energy loss between the elastic scattering events. This assumptions justifies using the analytical solution for charged particle transport in vacuum. To evaluate the validity of this assumption, the EGSnrc electron transport code was modified to output the elastic scattering mean free path (MFP) and the energy lost by the electron over the length of the MFP. The energy loss is determined using the EGSnrc fourth-order Runge-Kutta method, which numerically integrates over the linear energy loss along the length of the step.

Fig. C.1 provides the electron elastic mean free path as calculated by EGSnrc for three materials. Over these ranges, the fractional energy loss is determined by calculating the kinetic energy lost over the step divided by the initial kinetic energy of the electron. Fig. C.2 shows that, at most, the energy loss is a little over $6 \%$ for a $1 \mathrm{keV}$ electron in water, and with increasing energy the energy loss quickly becomes negligible. In the context of the magnetic field transport algorithm, these results justify the assumption is that little energy is lost over the SS step.

These calculations were performed with an AE, lower particle cut-off energy used for the cross-section calculation, of $0.512 \mathrm{MeV}$. Increasing AE to a value of $0.711 \mathrm{MeV}$, roughly $200 \mathrm{keV}$ above the rest energy of the electron, produced slight increases of the percent energy loss. As AE is increased, the elastic scattering MFP decreases slightly 
and the stopping power increases (producing an overall increase in the fractional energy loss). The change in the MFP is due to the inclusion of spin-effects, and when this option is turned off the MFPs do no vary with energy (although the stopping powers still do). For a $1 \mathrm{MeV}$ electron changing AE from $0.512 \mathrm{MeV}$ to $0.711 \mathrm{MeV}$ changed the percent energy loss from $0.013 \%$ to $0.017 \%$, from $0.015 \%$ to $0.020 \%$, and from $0.013 \%$ to $0.018 \%$, for graphite, water, and air, respectively. Even at larger AE values the energy loss is negligible in the context of the magnetic field algorithm.

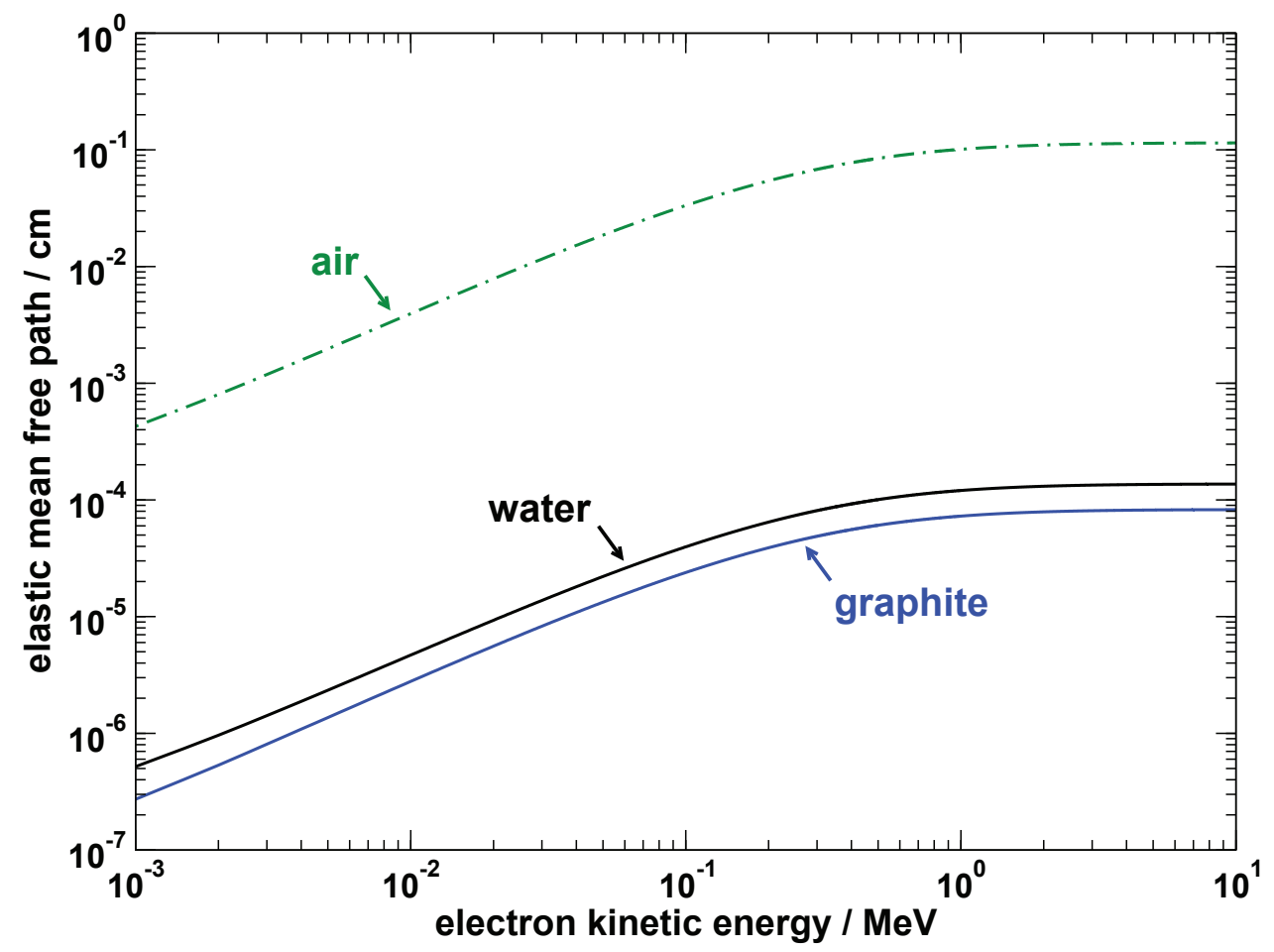

Figure C.1: Electron mean free path as a function of energy for air, water, and graphite. 


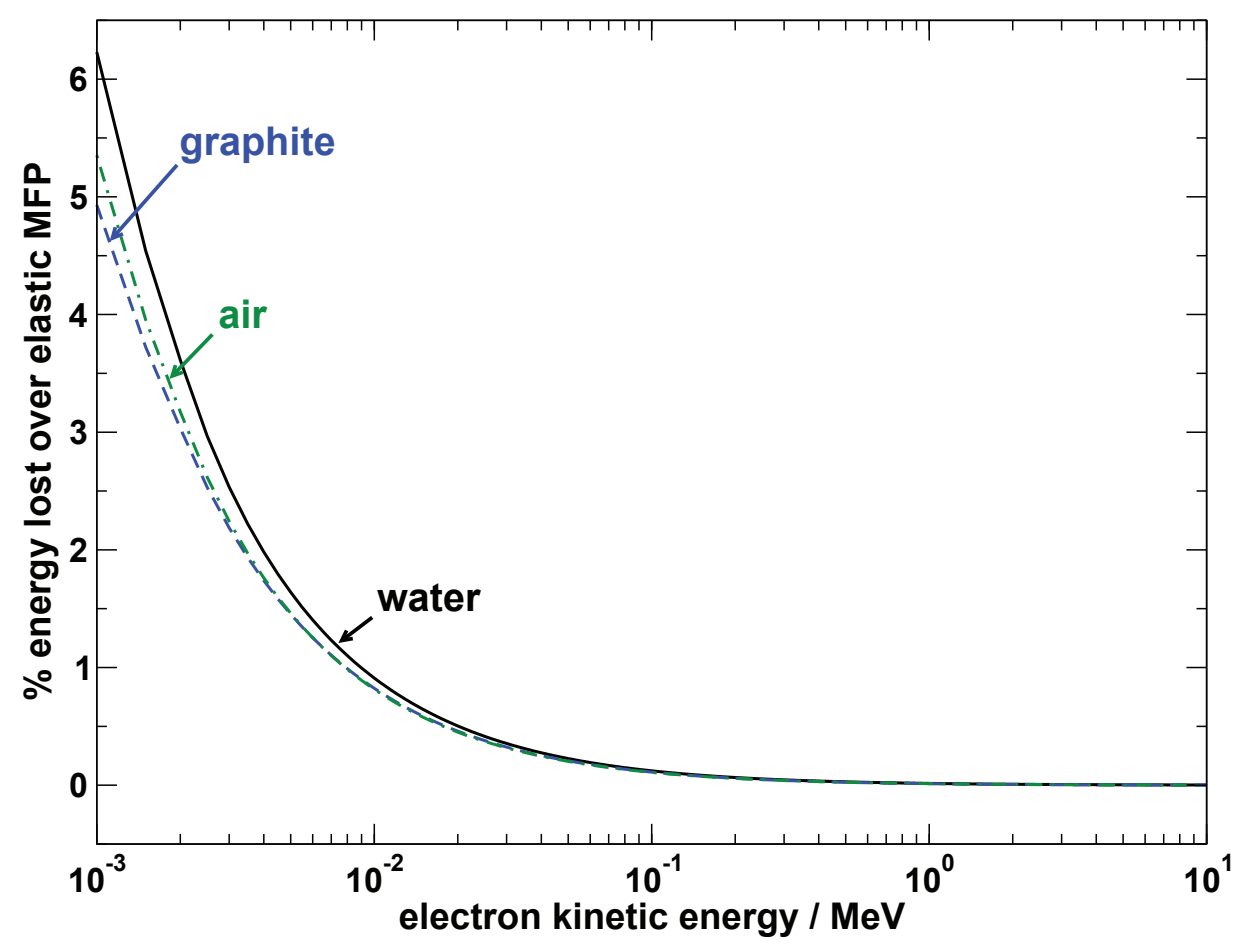

Figure C.2: Percent of kinetic energy lost per mean free path as a function of energy for air, water, and graphite. AE is set to $0.512 \mathrm{MeV}$. 


\section{References}

[1] Canadian Cancer Societys Advisory Committee on Cancer Statistics, Canadian Cancer Statistics, Canadian Cancer Society; (2017). (p 1)

[2] K. Otto, Volumetric modulated arc therapy: IMRT in a single gantry arc, Med. Phys. 35, $310-317$ (2008). (p 1)

[3] T. R. Mackie, T. Holmes, S. Swerdloff, P. Reckwerdt, J. O. Deasy, J. Yang, B. Paliwal, and T. Kinsella, Tomotherapy: A new concept for the delivery of dynamic conformal radiotherapy, Med. Phys. 20, 1709 - 1719 (1993). (p 1)

[4] Jaffray, David A and Siewerdsen, Jeffrey H and Wong, John W and Martinez, Alvaro A, Flat-panel cone-beam computed tomography for image-guided radiation therapy, International Journal of Radiation Oncology* Biology* Physics 53, 13371349 (2002). (p 2)

[5] W. Kilby, J. Dooley, G. Kuduvalli, S. Sayeh, and C. Maurer Jr, The CyberKnife@ robotic radiosurgery system in 2010, Technology in cancer research \& treatment 9, 433-452 (2010). (p 2)

[6] S. Mutic and J. F. Dempsey, The ViewRay System: Magnetic Resonance-Guided and Controlled Radiotherapy, Seminars in Radn. Oncol. 24, 196 - 199 (2014). (p 2)

[7] B. W. Raaymakers, A. J. E. Raaijmakers, A. N. T. J. Kotte, D. Jette, and J. J. W. Lagendijk, Integrating a MRI scanner with a 6 MV radiotherapy accelerator: dose deposition in a transverse magnetic field, Phys. Med. Biol. 49, 4109-4118 (2004). (pp 2 and 3 )

[8] A. J. E. Raaijmakers, B. W. Raaymakers, and J. J. W. Lagendijk, Integrating a MRI scanner with a $6 \mathrm{MV}$ radiotherapy accelerator: dose increase at tissue-air interfaces in a lateral magnetic field due to returning electrons, Phys. Med. Biol. 50, $1363-1376$ (2005). (pp 2 and 45)

[9] A. J. E. Raaijmakers, B. W. Raaymakers, and J. J. W. Lagendijk, Experimental verification of magnetic field dose effects for the MRI-accelerator, Phys. Med. Biol. $\mathbf{5 2}, 4283-4291$ (2007). (p 2)

[10] B. M. Oborn, P. E. Metcalfe, M. J. Butson, and A. B. Rosenfeld, High resolution entry and exit Monte Carlo dose calculations from a linear accelerator $6 \mathrm{MV}$ beam 
under the influence of transverse magnetic fields, Med. Phys. 36, 3549 - 3559 (2009). (p 2)

[11] B. G. Fallone, B. Murray, S. Rathee, T. Stanescu, S. Steciw, S. Vidakovic, E. Blosser, and D. Tymofichuk, First MR images obtained during megavoltage photon irradiation from a prototype integrated linac-MR system, Med. Phys. 36, 2084-2088 (2009). (p 2)

[12] C. Kirkby, B. Murray, S. Rathee, and B. G. Fallone, Lung dosimetry in a linacMRI radiotherapy unit with a longitudinal magnetic field, Med. Phys. 37, 4722 4732 (2010). (pp 2, 3, 13, 18, and 45)

[13] X. A. Li, L. Reiffel, J. Chu, and S. Naqvi, Conformal photon-beam therapy with transverse magnetic fields: A Monte Carlo study, Med. Phys. 28, 127 - 133 (2001). (p 3)

[14] D. Jette, Magnetic fields with photon beams: Monte Carlo calculations for a model magnetic field, Med. Phys. 27, 2726-2738 (2000). (p 3)

[15] C. Kirkby, T. Stanescu, S. Rathee, M. Carlone, B. Murray, and B. G. Fallone, Patient dosimetry for hybrid MRI-radiotherapy systems, Med. Phys. 35, 1019 1027 (2008). (p 3)

[16] B. M. Oborn, P. E. Metcalfe, M. J. Butson, and A. B. Rosenfeld, Monte Carlo characterization of skin doses in $6 \mathrm{MV}$ transverse field MRI-linac systems: Effect of field size, surface orientation, magnetic field strength, and exit bolus, Med. Phys. $37,5208-5217$ (2010). (pp 3 and 45)

[17] A. F. Bielajew, The effect of strong longitudinal magnetic fields on dose deposition from electron and photon beams, Med. Phys. 20, 1171 - 1179 (1993). (p 3)

[18] A. J. E. Raaijmakers, B. W. Raaymakers, and J. J. W. Lagendijk, Magneticfield-induced dose effects in MR-guided radiotherapy systems: dependence on the magnetic field strength, Phys. Med. Biol. 53, 909 - 923 (2008). (pp 3 and 45)

[19] M. Reynolds, B. G. Fallone, and S. Rathee, Technical Note: Response measurement for select radiation detectors in magnetic fields, Med. Phys. 42, $2837-2840$ (2015). (pp 3 and 20)

[20] M. Reynolds, B. G. Fallone, and S. Rathee, Dose response of selected ion chambers in applied homogeneous transverse and longitudinal magnetic fields, Med. Phys. 40, 042102, 7pp (2013). (pp 3, 20, 54, and 56)

[21] IAEA, Absorbed Dose Determination in External Beam Radiotherapy: An International Code of Practice for Dosimetry Based on Standards of Absorbed Dose to Water, volume 398 of Technical Report Series, IAEA, Vienna, 2001. (pp 4 and 9)

[22] P. R. Almond, P. J. Biggs, B. M. Coursey, W. F. Hanson, M. S. Huq, R. Nath, and D. W. O. Rogers, AAPM's TG-51 protocol for clinical reference dosimetry of high-energy photon and electron beams, Med. Phys. 26, 1847 - 1870 (1999). (pp $4,5,6$, and 7 ) 
[23] M. R. McEwen, L. A. DeWerd, G. S. Ibbott, D. S. Followill, D. W. O. Rogers, S. M. Seltzer, and J. P. Seuntjens, Addendum to the AAPM's TG-51 protocol for clinical reference dosimetry of high-energy photon beams, Med. Phys. 41, 041501(20pp) (2014). (pp 4, 7, 81, 84, 85, 88, 90, 93, and 100)

[24] I. Kawrakow and D. W. O. Rogers, The EGSnrc System, a status report., in Advanced Monte Carlo for Radiation Physics, Particle Transport Simulation and Applications: Proc. of the Monte Carlo 2000 Meeting Lisbon, edited by A. Kling, F. Barao, M. Nakagawa, L. Távora, and P. Vaz, pages 135 - 140, Springer, Berlin, 2001. (pp 9, 96, and 99)

[25] G. Xiong and D. W. O. Rogers, Relationship between \%dd(10) $\times$ and stoppingpower ratios for flattening filter free accelerators: A Monte Carlo study, Med. Phys. 35, $2104-2109$ (2008). (p 9)

[26] D. J. O'Brien, D. A. Roberts, G. S. Ibbott, and G. O. Sawakuchi, Reference dosimetry in magnetic fields: formalism and ionization chamber correction factors, Med. Phys. 43, 4915 - 4927 (2016). (pp 11, 54, 68, 69, 91, 95, 97, 98, and 99)

[27] C K Spindeldreier et al., Radiation dosimetry in magnetic fields with Farmer-type ionization chambers: determination of magnetic field correction factors for different magnetic field strengths and field orientations, Phys. Med. Biol. 62, 6708-6728 (2017). (pp 11, 12, 71, 81, and 85)

[28] V. N. Malkov and D. W. O. Rogers, Sensitive volume effects on Monte Carlo calculated ion chamber response in magnetic fields, Med. Phys. , n/a-n/a (2017). (pp 12, 71, and 81)

[29] V. N. Malkov and D. W. O. Rogers, Charged particle transport in magnetic fields in EGSnrc, Med. Phys. 43, 4447 - 4458 (2016). (pp 12, 54, and 81)

[30] S. L. Hackett, B. van Asselen, J. W. H. Wolthaus, J. G. M. Kok, S. J. Woodings, J. J. W. Lagendijk, and B. W. Raaymakers, Consequences of air around an ionization chamber: Are existing solid phantoms suitable for reference dosimetry on an MR-linac?, Med. Phys. 43, 3961 - 3968 (2016). (p 12)

[31] J. Agnew, F. O'Grady, R. Young, S. Duane, and G. J. Budgell, Quantification of static magnetic field effects on radiotherapy ionization chambers, Phys. Med. Biol. 62, $1731-1743$ (2017). (pp 12, 54, 55, 56, 60, and 62)

[32] I. Kawrakow, E. Mainegra-Hing, D. W. O. Rogers, F. Tessier, and B. R. B. Walters, The EGSnrc Code System: Monte Carlo simulation of electron and photon transport, NRC Technical Report PIRS-701 v4-2-32, National Research Council Canada, Ottawa, Canada. http://www.nrccnrc.gc.ca/eng/solutions/advisory/egsnrc/download_egsnrc.html, 2011. (pp 12 and 16)

[33] I. Kawrakow, Accurate condensed history Monte Carlo simulation of electron transport. I. EGSnrc, the new EGS4 version, Med. Phys. 27, 485 - 498 (2000). (pp 12, 16, and 26) 
[34] J. Wulff, J. T. Heverhagen, and K. Zink, Monte-Carlo-based perturbation and beam quality correction factors for thimble ionization chambers in high-energy photon beams, Phys. Med. Biol. 53, 2823 - 2836 (2008). (pp 13 and 54)

[35] F. Salvat, J. M. Fernandez-Varea, J. Baro, and J. Sempau, PENELOPE, a code system for Monte Carlo simulation of electron and photon transport, University of Barcelona Report (2003). (pp 13 and 108)

[36] J. S. Bull, H. G. Hughes, P. L. Walstrom, J. D. Zumbro, and N. V. Mokhov, Magnetic field tracking with MCNP5, Radiat. Prot. Dosim. 116, 307 - 311 (2005). (p 13)

[37] I. Kawrakow and D. W. O. Rogers, The EGSnrc Code System: Monte Carlo simulation of electron and photon transport, Technical Report PIRS-701, National Research Council Canada, Ottawa, Canada, 2006. (p 13)

[38] I. Kawrakow, Accurate condensed history Monte Carlo simulation of electron transport. II. Application to ion chamber response simulations, Med. Phys. 27, $499-513$ (2000). (pp 13 and 29)

[39] Y. M. Yang and B. Bednarz, Consistency evaluation between EGSnrc and Geant4 charged particle transport in an equilibrium magnetic field, Phys. Med. Biol. 58, N47 - N58 (2013). (pp 13, 18, 49, and 108)

[40] A. F. Bielajew, Electron Transport in $\vec{E}$ and $\vec{B}$ Fields, in Monte Carlo Transport of Electrons and Photons, edited by T. M. Jenkins, W. R. Nelson, A. Rindi, A. E. Nahum, and D. W. O. Rogers, pages 421 - 434, Plenum Press, New York, 1988. (pp 13, 17, 19, 20, 23, and 108)

[41] J. A. de Pooter, L. A. de Prez, and H. Bouchard, Application of an adapted Fano cavity test for Monte Carlo simulations in the presence of B-fields, Phys. Med. Biol. 60, 9313 - 9327 (2015). (pp 13 and 27)

[42] I Meijsing et al., Dosimetry for the MRI accelerator: the impact of a magnetic field on the response of a Farmer NE2571 ionization chamber, Phys. Med. Biol. 54, $2993-3002$ (2009). (pp 13, 33, 34, 35, 36, 42, 54, 56, and 81)

[43] E. Poon, J. S. Seuntjens, and F. Verhaegen, Consistency test of the electron transport algorithm in the GEANT4 Monte Carlo code, Phys. Med. Biol. 50, 681 - 694 (2005). (p 13)

[44] S. Elles, V. N. Ivanchenko, M. Maire, and L. Urban, Geant4 and Fano cavity test: where are we?, J of Phys: Conf Ser 102, 012009 (2008). (p 13)

[45] J. P. Archambault and E. Mainegra-Hing, Comparison between EGSnrc, Geant4, MCNP5 and Penelope for mono-energetic electron beams, Phys. Med. Biol. 60, $4951-4962$ (2015). (p 13)

[46] R. Burden and J. Farires, Numerical Analysis, 9-nd Ed, Boston, USA (2011). (p 21) 
[47] U. Fano, Note on the Bragg-Gray cavity principle for measuring energy dissipation, Radiat. Res. 1, 237 - 240 (1954). (p 26)

[48] J. Sempau and P. Andreo, Configuration of the electron transport algorithm of PENELOPE to simulate ion chambers, Phys. Med. Biol. 51, 3533 - 3548 (2006). (pp 26 and 27)

[49] H. Bouchard and A. Bielajew, Lorentz force correction to the Boltzmann radiation transport equation and its implications for Monte Carlo algorithms, Phys. Med. Biol. 60, 4963 - 4971 (2015). (p 27)

[50] H. Bouchard, J. de Pooter, A. Bielajew, and S. Duane, Reference dosimetry in the presence of magnetic fields: conditions to validate Monte Carlo simulations, Phys. Med. Biol. 60, 6639 - 6654 (2015). (p 27)

[51] D. J. La Russa and D. W. O. Rogers, Accuracy of Spencer-Attix cavity theory and calculations of fluence correction factors for the air kerma formalism, Med. Phys. 36, $4173-4183$ (2009). (p 29)

[52] J. Wulff, K. Zink, and I. Kawrakow, Efficiency improvements for ion chamber calculations in high energy photon beams, Med. Phys. 35, 1328- 1336 (2008). (pp 33,55 , and 56)

[53] D. Sheikh-Bagheri and D. W. O. Rogers, Monte Carlo calculation of nine megavoltage photon beam spectra using the BEAM code, Med. Phys. 29, $391-402$ (2002). (pp 34 and 96)

[54] D. J. La Russa, M. McEwen, and D. W. O. Rogers, An experimental and computational investigation of the standard temperature-pressure correction factor for ion chambers in kilovoltage x rays, Med. Phys. 34, 4690 - 4699 (2007). (p 34)

[55] D. J. OBrien, S. L. Hackett, B. van Asselen, G. Ibbott, B. W. Raaymakers, G. O. Sawakuchi, and J. W. H. Wolthaus, TH-CD-304-08: Small Air-Gaps Affect the Response of Ionization Chambers in the Presence of a 1.5 T Magnetic Field, Med. Phys. 42, 3724 - 3724 (2015). (p 37)

[56] S. Standring, Gray's anatomy: the anatomical basis of clinical practice, Elsevier Health Sciences, 2015. (p 44)

[57] P. N. McDermott, T. He, and A. DeYoung, Dose calculation accuracy of lung planning with a commercial IMRT treatment planning system, J of App Clin Med Phys 4, 341 - 351 (2003). (p 44)

[58] G. Cranmer-Sargison, W. A. Beckham, and I. A. Popescu, Modeling an extreme water lung interface using a single pencil beam algorithm and the Monte Carlo method, Phys. Med. Biol. 49, 15571567 (2004). (p 44)

[59] H. Saitoh, T. Fujisaki, R. Saskai, and E. Kunieda, Dose distribution of narrow beam irradiation for small lung tumor, Int. J. Radiat. Oncol., Biol., Phys. 53(5), 13801387 (2002). (p 44) 
[60] ICRU, Tissue Substitutes in Radiation Dosimetry and Measurements, ICRU Report 44, ICRU, Washington D.C., 1989. (p 44)

[61] K. Babcock and N. Sidhu, The effects of dose calculation resolution on dose accuracy for radiation therapy treatments of the lung. Part II. A comparison of dose distributions from an explicit lung model to dose distributions derived from a CT representation, Med. Phys. 37, 687 - 693 (2010). (pp 44 and 53)

[62] C. Bauer, M. Eberlein, and R. R. Beichel, Graph-based airway tree reconstruction from chest CT scans: evaluation of different features on five cohorts, Medical Imaging, IEEE Transactions on 34, 1063-1076 (2015). (p 45)

[63] J. Pu, S. Gu, S. Liu, S. Zhu, D. Wilson, J. M. Siegfried, and D. Gur, CT based computerized identification and analysis of human airways: a review, Medical physics 39, 2603-2616 (2012). (p 45)

[64] S. R. Mahdavi, A. D. Esmaeeli, M. Pouladian, A. S. Monfared, D. Sardari, and S. Bagheri, Breast dosimetry in transverse and longitudinal field MRI-Linac radiotherapy systems, Med. Phys. 42, 925-936 (2015). (p 45)

[65] S. B. Ahmad, A. Sarfehnia, M. R. Paudel, A. Kim, S. Hissoiny, A. Sahgal, and B. Keller, Evaluation of a commercial MRI Linac based Monte Carlo dose calculation algorithm with GEANT4, Med. Phys. 43, 894 - 907 (2016). (pp 45, 55, 68, and 96)

[66] M. J. Menten, M. F. Fast, S. Nill, C. P. Kamerling, F. McDonald, and U. Oelfke, Lung stereotactic body radiotherapy with an MR-linac - Quantifying the impact of the magnetic field and real-time tumor tracking, Radiother. Oncol. 119, 461 466 (2016). (p 45)

[67] K. Babcock and N. Sidhu, The effects of dose calculation resolution on dose accuracy for radiation therapy treatments of the lung. Part I. A Monte Carlo model of the lung, Med. Phys. 37, 675 - 686 (2010). (p 45)

[68] J. Seuntjens, A. Van der Plaetsen, H. Thierens, and M. Piessens, Comparison of measured and calculated dose distributions in lung after electron beam treatment of the chest wall, Med. Phys. 21, 1959 - 1968 (1994). (p 45)

[69] B. R. Muir and D. W. O. Rogers, Monte Carlo calculations of $k_{Q}$, the beam quality conversion factor, Med. Phys. 37, 5939 - 5950 (2010). (pp 54, 57, 80, 84, 88 , and 89)

[70] J. R. Miller, B. D. Hooten, J. A. Micka, and L. A. DeWerd, Polarity effects and apparent ion recombination in microionization chambers, Med. Phys. 43, 21412152 (2016). (pp 54, 55, 56, 61, and 71)

[71] C. K. Ross, Comments on 'Ionization chamber volume determination and quality assurance using micro-CT imaging', Phys. Med. Biol. 54, L23 - 27 (2009). (pp 54, $55,56,61$, and 71) 
[72] D. J. Butler, A. W. Stevenson, T. E. Wright, P. D. Harty, J. Lehmann, J. Livingstone, and J. C. Crosbie, High spatial resolution dosimetric response maps for radiotherapy ionization chambers measured using kilovoltage synchrotron radiation, Phys. Med. Biol. 60, 8625 - 8641 (2015). (p 55)

[73] B. R. Muir, M. R. McEwen, and D. W. O. Rogers, Measured and Monte Carlo calculated $k_{Q}$ factors: accuracy and comparison, Med. Phys. 38, $4600-4609$ (2011). (pp 55, 89, 93, and 94)

[74] G. Mora, A. Maio, and D. W. O. Rogers, Monte Carlo simulation of a typical ${ }^{60}$ Co therapy source, Med. Phys. 26, 2494 - 2502 (1999). (pp 55, 68, and 96)

[75] B. R. Muir, M. R. McEwen, and D. W. O. Rogers, Beam quality conversion factors for parallel-plate ionization chambers in MV photon beams, Med. Phys. 39, 1618 - 1631 (2012). (pp 57 and 94)

[76] K. Smit, B. van Asselen, J. G. M. Kok, A. H. L. Aalbers, J. J. W. Lagendijk, and B. W. Raaymakers, Towards reference dosimetry for the MR-linac: magnetic field correction of the ionization chamber reading, Phys. Med. Biol. 58, 5945 - 5957 (2013). (p 68)

[77] M. Reynolds, S. Rathee, and B. G. Fallone, Technical Note: Ion chamber angular dependence in a magnetic field, Med. Phys. 44, 4322-4328 (2017). (pp 20 and 78)

[78] M. Dalaryd, T. Knoos, and C. Ceberg, Combining tissue-phantom ratios to provide a beam-quality specifier for flattening filter free photon beams, Med. Phys. 41, 111716(6pp) (2014). (p 84)

[79] ICRU, Stopping powers for electrons and positrons, ICRU Report 37, ICRU, Bethesda, MD., 1984. (pp 88 and 92)

[80] ICRU, Report 90: Key data for ionizing-radiation dosimetry: measurement standards and applications, J ICRU 14, 1-110 (2014). (pp 88, 92, and 93)

[81] K. Javedan et al., Monte Carlo comparison of superficial dose between flattening filter free and flattened beams, Physica Medica 30, 503-508 (2014). (p 96) 